\title{
A Hybrid Location Identification Method in Wireless Ad Hoc/Sensor Networks
}

\author{
By \\ Yifan Zhao, B. Sc. \\ Nanjing University \\ A thesis submitted to the Faulty of Graduate Studies and Research \\ In partial fulfillment of the requirements for the degree of

\section{Master of Applied Science} \\ Ottawa-Carleton Institute for Electrical Engineering \\ Department of Systems and Computer Engineering \\ Carleton University \\ Ottawa, Ontario, Canada \\ April 2008
}

(c) Copyright 2008, Yifan Zhao 


$\begin{array}{ll}\begin{array}{l}\text { Library and } \\ \text { Archives Canada }\end{array} & \begin{array}{l}\text { Bibliothèque et } \\ \text { Archives Canada }\end{array} \\ \begin{array}{l}\text { Published Heritage } \\ \text { Branch }\end{array} & \begin{array}{l}\text { Direction du } \\ \text { Patrimoine de l'édition }\end{array} \\ \begin{array}{l}\text { 395 Wellington Street } \\ \text { Ottawa ON K1A 0N4 } \\ \text { Canada }\end{array} & \begin{array}{l}\text { O95, rue Wellington } \\ \text { Ottawa ON K1A 0N4 } \\ \text { Canada }\end{array}\end{array}$

Your file Votre référence ISBN: 978-0-494-44066-7

Our file Notre référence

ISBN: 978-0-494-44066-7

NOTICE:

The author has granted a nonexclusive license allowing Library and Archives Canada to reproduce, publish, archive, preserve, conserve, communicate to the public by telecommunication or on the Internet, loan, distribute and sell theses worldwide, for commercial or noncommercial purposes, in microform, paper, electronic and/or any other formats.

The author retains copyright ownership and moral rights in this thesis. Neither the thesis nor substantial extracts from it may be printed or otherwise reproduced without the author's permission.
AVIS:

L'auteur a accordé une licence non exclusive permettant à la Bibliothèque et Archives Canada de reproduire, publier, archiver, sauvegarder, conserver, transmettre au public par télécommunication ou par l'Internet, prêter, distribuer et vendre des thèses partout dans le monde, à des fins commerciales ou autres, sur support microforme, papier, électronique et/ou autres formats.

L'auteur conserve la propriété du droit d'auteur et des droits moraux qui protège cette thèse. $\mathrm{Ni}$ la thèse ni des extraits substantiels de celle-ci ne doivent être imprimés ou autrement reproduits sans son autorisation.
In compliance with the Canadian

Privacy Act some supporting forms may have been removed from this thesis.

While these forms may be included in the document page count, their removal does not represent any loss of content from the thesis.
Conformément à la loi canadienne sur la protection de la vie privée, quelques formulaires secondaires ont été enlevés de cette thèse.

Bien que ces formulaires aient inclus dans la pagination, il n'y aura aucun contenu manquant.

\section{Canada}




\begin{abstract}
Location identification in Ad Hoc/Sensor networks is a process for estimating the location of a mobile node by using the information provided by other mobile nodes that know their own positions.

In this thesis, we propose a practical implementation of a location identification system using TDOA technology. In this system, only the basic assumptions that are acceptable in most types of Ad Hoc/Sensor networks are held, which means this system is implementable in most kinds of Ad Hoc/Sensor networks.

By introducing another location identification method into the system, some drawbacks to the TDOA system are resolved. More importantly, by employing the new theory, the location estimation accuracy of the proposed system is improved without costing extra resources.

Finally, simulations show that the Hybrid TDOA location estimation system proposed in this thesis performs better in different environments compared with the regular TDOA method.
\end{abstract}




\section{Acknowledgements}

Many thanks first go to my supervisors, Professor Lung and Professor Lambadaris, for their valuable guidance, thoughtful advice, and continuous support throughout my study and research work for this thesis. Finally, I am very grateful to my friends and my family for their direct or indirect contributions to this thesis. 


\section{Table of Contents}

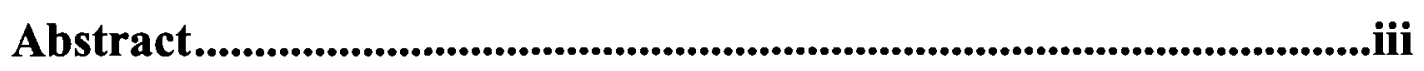

Acknowledgements ..................................................................................iv

Table of Contents ...........................................................................................

List of Figures...........................................................................................viii

List of Tables ..........................................................................................

List of Notations ................................................................................

List of Acronyms ............................................................................

Chapter 1 Introduction......................................................................13

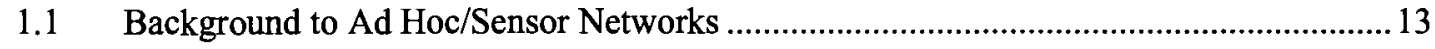

1.1.1 Ad Hoc/Sensor Network Characteristics....................................................... 13

1.1.2 Location Identification in Ad Hoc/Sensor Networks ........................................15

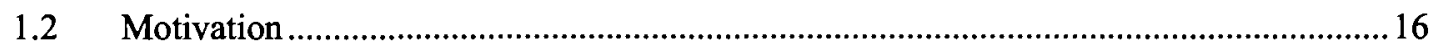

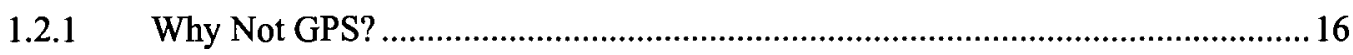

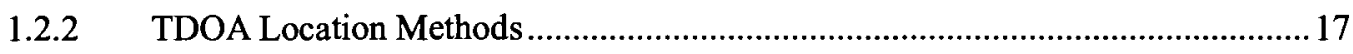

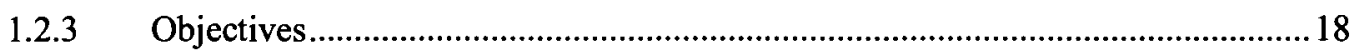

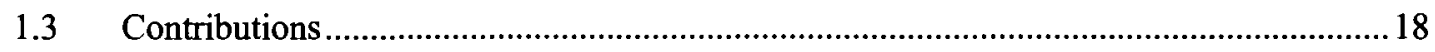

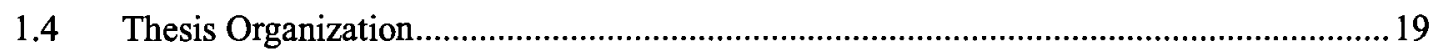

\section{Chapter 2 Survey of Location Identification methods .................20}

2.1 Categories of Location Identification Technology in Wireless Networks .........................20

2.1.1 Source of Measurement-based Classification.......................................................20

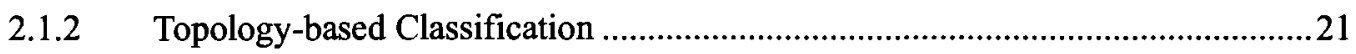

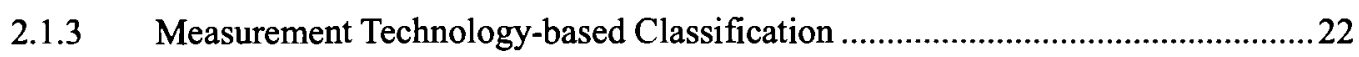

2.1.4 Overview of Current Wireless Location Identification Systems .............................32

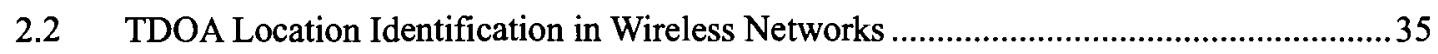

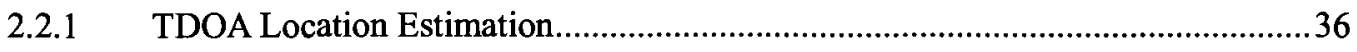

2.2.2 Improved TDOA in Wireless Ad Hoc/Sensor Networks .......................................39

Chapter 3 Hybrid TDOA Method 


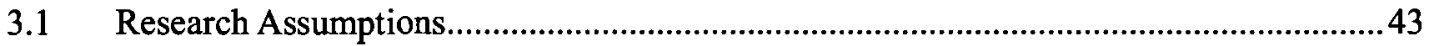

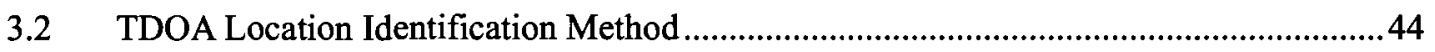

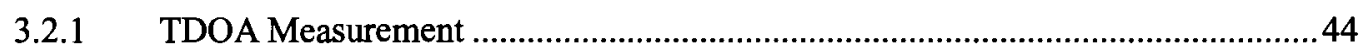

3.2.2 Nonlinear Equations Calculation.......................................................................46

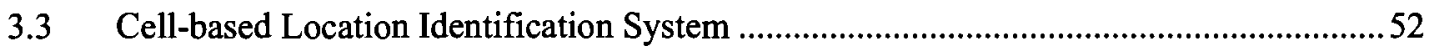

3.4 The Influence of Geometric Dilution of Precision in Location Identification Systems .... 54

3.4.1 Basic Idea of Geometric Dilution of Precision.....................................................5

3.4.2 Details of Geometric Dilution of Precision Influence ..........................................57

3.5 Hybrid TDOA Location Identification Method ...............................................................6

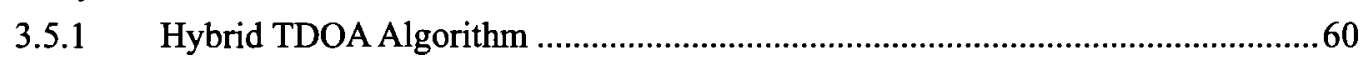

\section{Chapter 4 Simulation and Analysis .....................................................70}

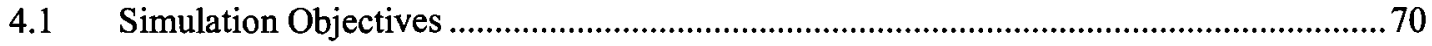

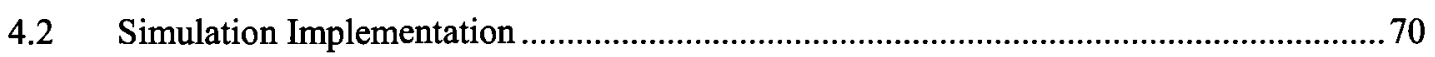

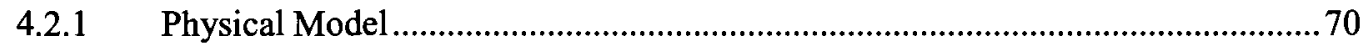

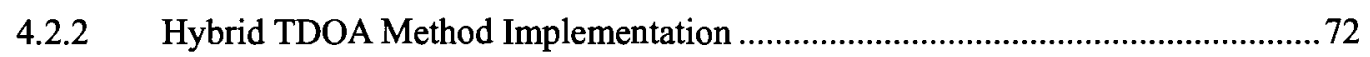

4.2.3 Regular LS TDOA Method Implementation .......................................................

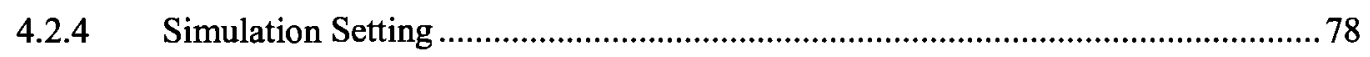

4.3 Performance of Regular TDOA and Hybrid TDOA in a Location Identification Process 80

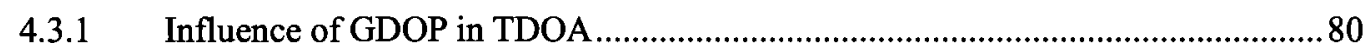

4.3.2 More than Three Reference Nodes in a Estimation Process.................................87

4.3.3 Influence of Noise in Estimation...................................................................98

4.4 Performance of TDOA and Hybrid TDOA Method in Entire Network............................. 106

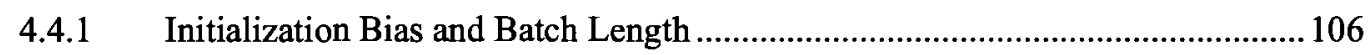

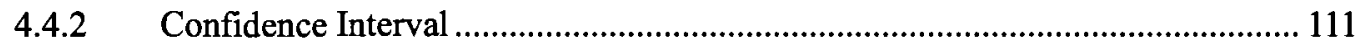

4.4.3 Performance vs. Number of Reference Nodes ................................................... 114

4.4.4 Performance vs. Density of Reference Nodes.................................................... 117

4.4.5 Performance vs. Density of Reference Node \& Noise Level .............................. 120

\section{Chapter 5 Conclusions and Future Work.....................................129}

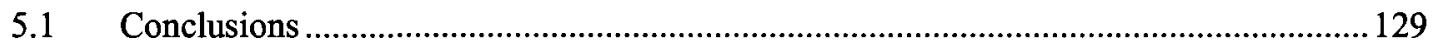

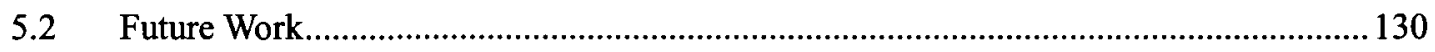

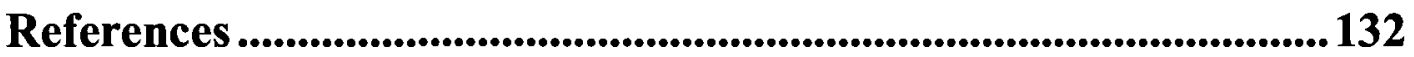

Appendix A: Triangulation ................................................................141

Appendix B: Cross-correlation Technique ........................................142

Appendix C: Simulation Implementation in NS2 ............................ 144

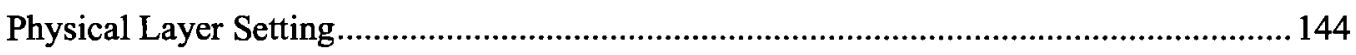


Appendix D: Hybrid TDOA Location Identification Method

Implementation Details ...............................................................150 


\section{List of Figures}

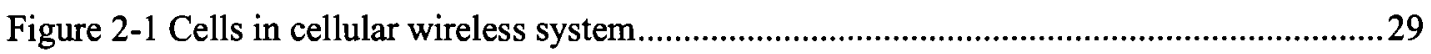

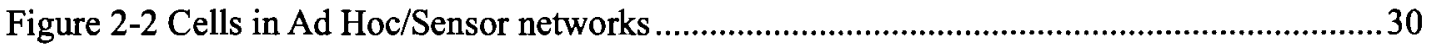

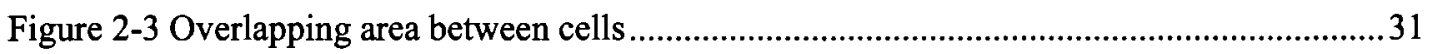

Figure 2-4 Cell area classification in Ad Hoc/Sensor network ...................................................... 32

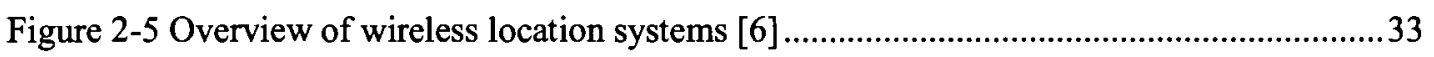

Figure 2-6 Comparison between estimation accuracy and wireless systems [26]........................34

Figure 2-7 Sketch of mobile stations (source nodes) localization.............................................. 41

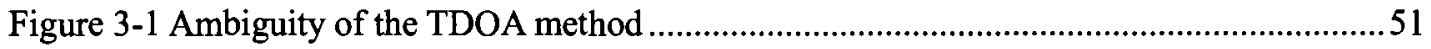

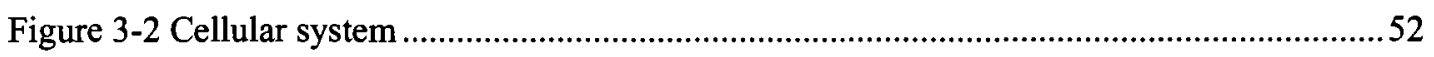

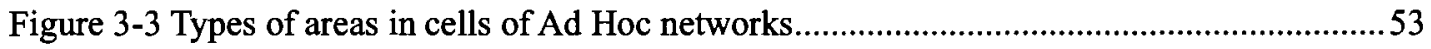

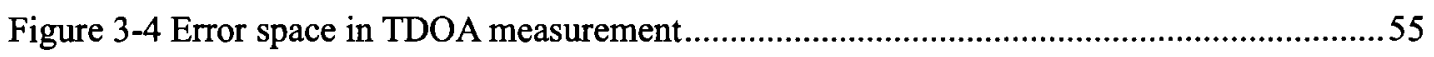

Figure 3-5 Error space in TDOA when relative positions have changed ....................................56

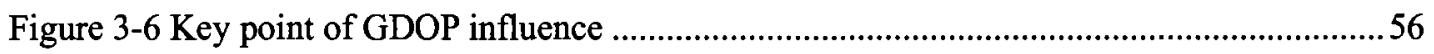

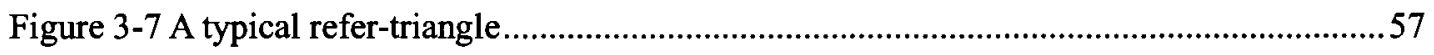

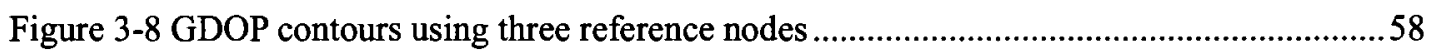

Figure 3-9 Normalized GDOP contours using three reference nodes ........................................59

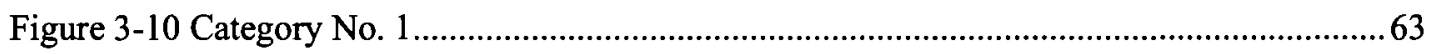

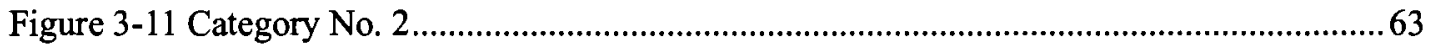

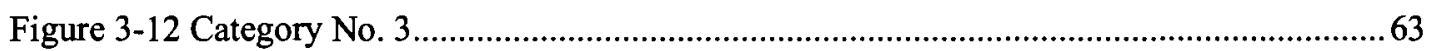

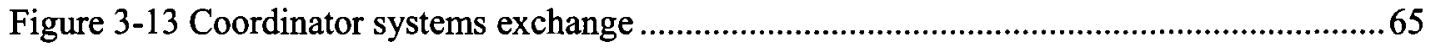

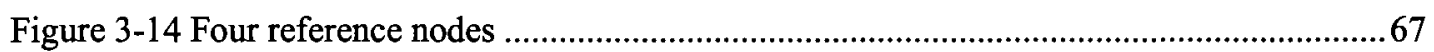

Figure 3-15 Candidate estimations in different refer-triangles....................................................67

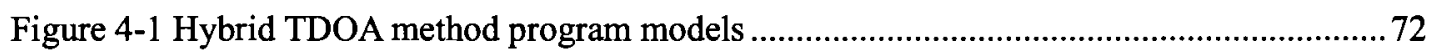

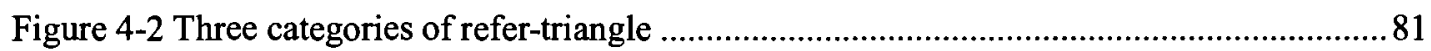

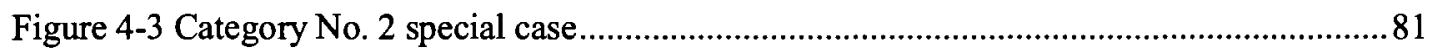

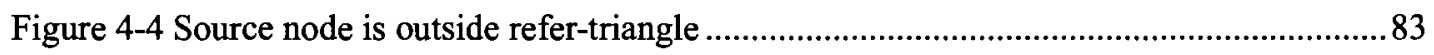

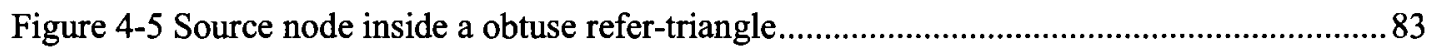

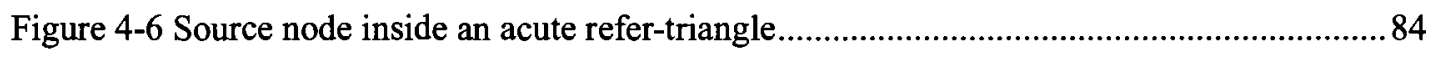

Figure 4-7 Error distance comparison of three categories.......................................................... 85

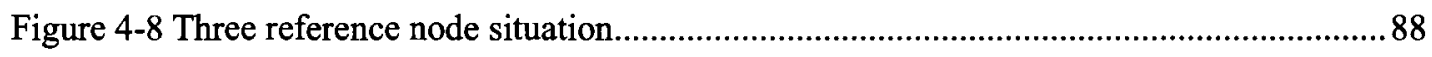

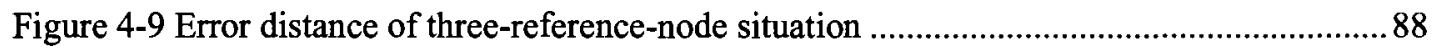

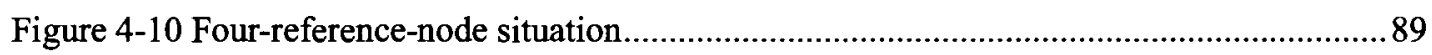

Figure 4-11 Error distance of four-reference-node situation........................................................... 89

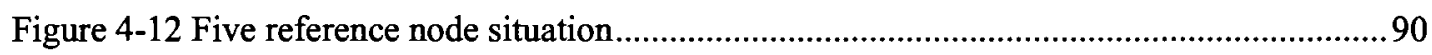

Figure 4-13 Error distance of five-reference-node situation .................................................... 90

Figure 4-14 Error distance comparison of different number of reference nodes............................90

Figure 4-15 Different refer-triangles in five-reference-node situation .........................................92

Figure 4-16 Refer-triangle classification in a five reference node situation...............................92 
Figure 4-17 Refer-triangle classification in a four reference node situation ..................................93

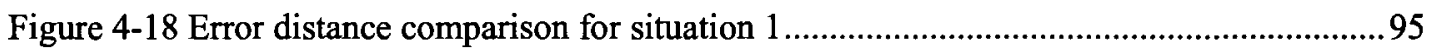

Figure 4-19 Error distance comparison for situation 2 .............................................................95

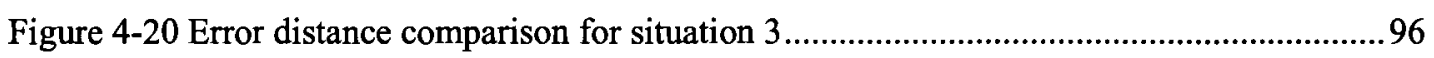

Figure 4-21 Mean of error distance comparison in three situations .............................................96

Figure 4-22 Standard deviation of error distance comparison in three situations ..........................97

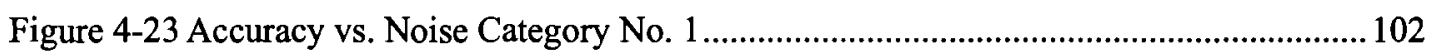

Figure 4-24 Successful estimation percentage vs. Noise Category No. 1 ................................... 102

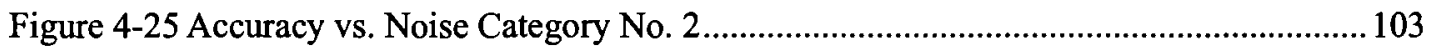

Figure 4-26 Successful estimation percentage vs. Noise Category No. 2 .................................. 103

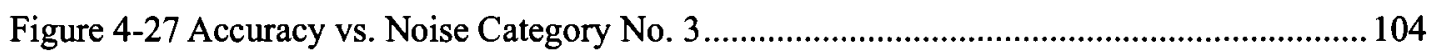

Figure 4-28 Successful estimation percentage vs. Noise Category No. 3 ................................. 104

Figure 4-29 Percentage of success estimation vs. noise level .................................................105

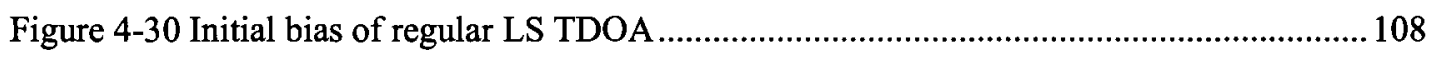

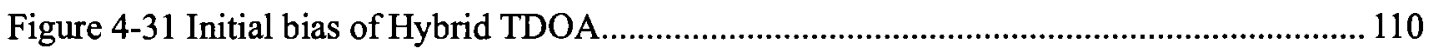

Figure 4-32 Long term performance of Reg. LS TDOA vs. Hybrid TDOA .............................. 115

Figure 4-33 Category No. 1 in all possible combinations ......................................................116

Figure 4-34 Long-term performance vs. density of reference nodes......................................... 119

Figure 4-35 Average number of reference nodes vs. density of reference nodes .......................... 119

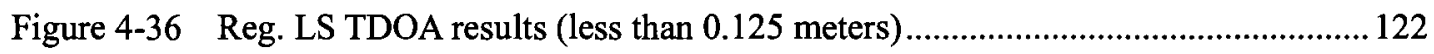

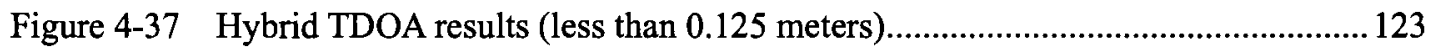

Figure 4-38 Reg. LS TDOA results (less than 0.175 meters) ............................................... 124

Figure 4-39 Hybrid TDOA results (less than 0.175 meters)................................................... 125

Figure 4-40 Results projected onto YZ plane (less than 0.125 meters) ........................................ 126

Figure 4-41 Results projected onto YZ plane (less than 0.175 meters) ........................................ 126

Figure 4-42 Results projected onto XZ plane (less than 0.125 meters)....................................... 127

Figure 4-43 Results projected onto XZ plane (less than 0.175 meters)........................................ 127

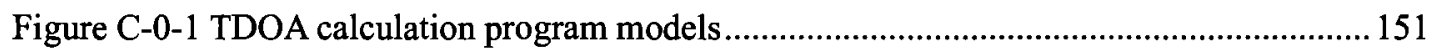

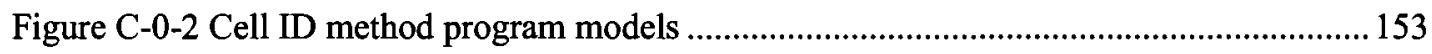

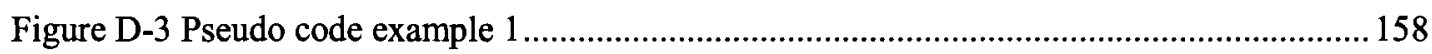

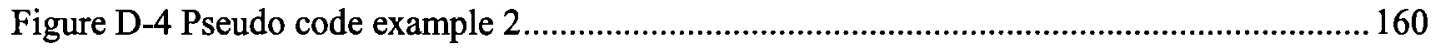




\section{List of Tables}

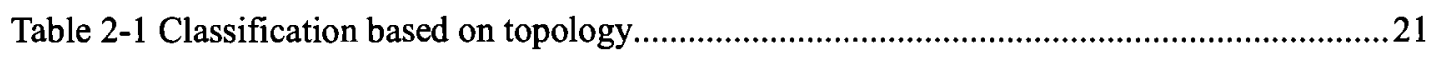

Table 2-2 Performance comparison of location identification systems ............................................. 34

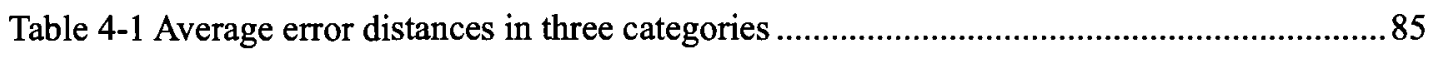

Table 4-2 Mean error distances and standard deviations in three categories..................................86

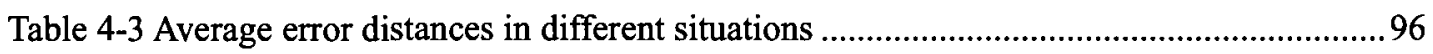

Table 4-4 Environmental variable 1 in NS2 noise model........................................................... 99

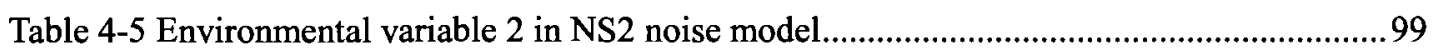

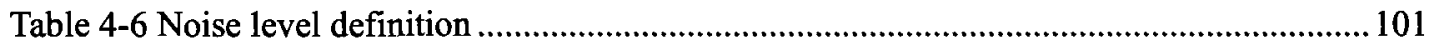

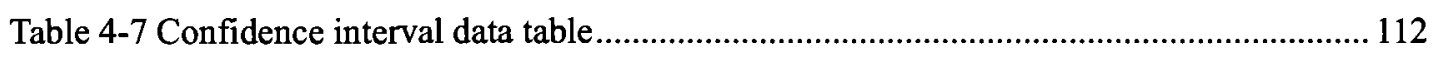

Table 4-8 Average category No. 1 in different number of reference nodes ................................. 117

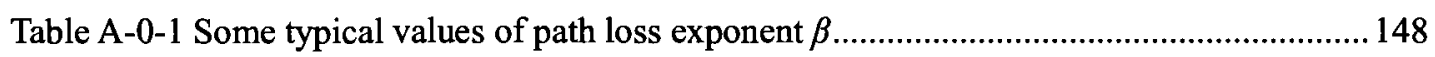

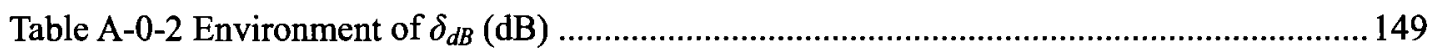




\section{List of Notations}

$M$

$C_{N}^{M}$

$\left(x_{i}, y_{i}\right)$

$R_{i}$

$R_{i, j}$

Dis $_{\text {Error }}$

$\left(x_{E}, y_{E}\right)$

$\Delta t_{i}$

$\left(x_{c}, y_{c}\right)$

$\mathrm{S} i-j$

$\sum_{i=1}^{N}\left(M_{i}\right)$

$X_{s}$
Reference nodes obtained the signal of source node

$\frac{M !}{N ! \times(M-N) !}$

Location of the $i^{\text {th }}$ mobile node

Range distance between source node and the $i^{\text {th }}$ mobile node

Range distance between the $i^{\text {th }}$ and $j^{\text {th }}$ mobile nodes

Error Distance

Source node's coordinates of estimated location

Time difference

Central point of cell

Slope of the line connecting points: $i$ and $j$

Sum of $M_{i}(i=1$ to $N)$

Coordinates of source node 


\section{List of Acronyms}

AOA

BTS

CDMA

CPU

DisError

$\mathrm{DaC}$

DOA

DECT

FCC

GDOP

GPS

GSM

LOS

LS

MS

MAC

MANet

NS2

NAG

NLSF

NLOS

OpNet

PCS

QoS

RF

RTOF

RSSI

RSS

TDOA

TOA

TTL

TCL

UWB

WAF

WLAN
Angle of Arrival

Base Transceiver Station

Code Division Multiple Access

Central Processing Unit

Error distance

Divide and Conquer

Direction of Arrival

Digital Enhanced Cordless Telecommunication

The United States Federal Communications Commission

Geometric Dilution of Precision

Global Positioning System

Global System for Mobile Communications

Line-of-Sight

Least Square

Mobile Station

Media Access Control

Mobile Ad Hoc Network

Network Simulator Version 2

Numerical Algorithm Group

Nonlinear Least Square Fitting

Non-Line-of-Sight

Optimized Network Evaluation Tool

Personal Communication Services

Quality of Service

Radio frequency

Round Trip Time of Flight

Received Signal Strength Indication

Really Simple Syndication

Time Difference of Arrival

Time of Arrival

Time to Live

Tool Command Language

Ultra Wide Band

Wall Attenuation Factor

Wireless Local Area Network 


\section{Chapter 1 Introduction}

\subsection{Background to Ad Hoc/Sensor Networks}

A wireless mobile Ad Hoc/Sensor network is a self-configurable network of mobile nodes that are connected by wireless links, with each node in the network acting as both router and host. The union of such nodes forms an arbitrary topology and the system remains organized and functional even when participating nodes are added, removed, or moved randomly. Because the network's wireless topology may change rapidly and unpredictably, new sets of problems unknown to traditional wireless networks arise.

Before the emergence of Ad Hoc/Sensor networks, wireless networks were supported with the help of fixed infrastructure. In contrast to infrastructure-based wireless networks, all nodes in an Ad Hoc/Sensor network can be mobile and connected dynamically in an arbitrary manner. Ad Hoc/Sensor networks can be deployed rapidly and used in places where a fixed infrastructure is not provided or the environment does not permit the installation of a fixed infrastructure.

\subsubsection{Ad Hoc/Sensor Network Characteristics}

Compared to today's popular wireless networks, such as GSM and CDMA, no fixed server or master is connected to an Ad Hoc/Sensor network infrastructure that provides wireless channels to mobile terminals [1]. In Ad Hoc/Sensor networks, every node acts as 
a router, relying on other nodes to forward packets, maintain communications, and find routes. Such networks may be autonomous, dynamic, and/or purpose-specific, e.g., emergency rescue teams. Therefore, Ad Hoc/Sensor networks are significantly different from traditional networks. This section highlights some of those key differences.

Normally, nodes in Ad Hoc/Sensor networks are less powerful than fixed network terminals. These nodes are likely characterized by low CPU speed, limited battery life, and small memory size. Such limitations significantly limit the capability of a node.

Routing decisions in Ad Hoc/Sensor networks are made by giving consideration to a greater number of factors than in a traditional wired routing framework. These factors include topology, route prioritization, flooding of route-requests, avoiding signal conflicts, and optimal utilization of available bandwidth.

Furthermore, Ad Hoc/Sensor networks are difficult to control and manage, including for activities like admission control, power management, and QoS control [2]. The difficulty arises from the fact that the control and management has to be distributed among all the nodes in the network, because each node takes the responsibility of forwarding packets to other nodes and maintaining the routes. Additionally, all nodes perform functions such as security and route policy management. Further difficulties regarding management arise from the unpredictably changing topology and unpredictable movements.

In summary, many technical issues must be solved before Ad Hoc/Sensor networks become readily preferred for most practical applications. 


\subsubsection{Location Identification in Ad Hoc/Sensor Networks}

Location identification is one of the most interesting features of wireless systems. In cellular wireless systems, e.g., in Global System for Mobile communications (GSM) and the third generation of cellular wireless network (CDMA), the United States Federal Communications Commission (FCC) required that by 2001, all mobile communications network operators should be able to accurately locate mobile callers requesting emergency assistance via 911 [3]. Thus, there has been significant activity among cellular and cellular/personal communication services (PCS) operators in terms of examining location identification in wireless systems. One of the major requirements of such systems is that no extra equipment is needed on mobile stations (cell phones); all location identification processes are operated on the network side. Recently, many commercial location identification systems for cellular wireless systems have also been produced [4, $5]$.

For Ad Hoc/Sensor networks, location identification is an even more attractive feature than in cellular wireless systems [6]. In sensor networks, recent technological developments have made an extremely small, cheap, and smart sensor node possible. These nodes can measure the environment, and collect, pre-calculate, and aggregate sensed data to provide continuous and spatially dense observations for further research. In such large sensor network systems, we need nodes to be aware of their own locations in various environments [6]. In Ad Hoc networks, the location identification service is 
also important; e.g., to identify the location of a wounded soldier on the battlefield, or to find the location of a rescue team in a disaster area.

In this research, the mobile unit that needs to find its own location is called the source node, and the mobile unit that knows its own location and has the ability to provide location identification measurements is called the reference node.

\subsection{Motivation}

As described above, location identification is a very valuable feature in wireless networking research. The following describes the challenges and objectives of location identification in ad hoc/sensor networks.

\subsubsection{Why Not GPS?}

One of the most straightforward solutions for the location identification system is to employ the Global Positioning System (GPS). To use GPS, each mobile unit may need to install a complete or a partial GPS receiver. However, this is not an attractive option for all situations, due to a number of factors. First, the additional GPS equipment would increase the size and weight of the mobile unit, and, of course, increase the cost of each mobile unit. Moreover, the GPS receiver would also lead to an additional drain on the power supply in the mobile unit, and in some Ad Hoc/Sensor network situations, recharging a mobile unit is impossible, thus higher power consumption results in a shorter lifetime for the mobile unit, which is highly undesirable. In addition, the mobile 
unit's antenna would need to be redesigned, because GPS operates in the $L$-band, which is seldom used in Ad Hoc/Sensor network frequencies [10]. Another negative factor is the GPS receiver's "warm-up" time. A GPS receiver may take at least one to one and a half minutes or even longer, depending on the design, to start giving readings after being turned on $[11,12]$. This would be critical in some time-sensitive applications. Lastly, a GPS receiver needs to have at least four satellites visible at all times, which is difficult in some places, such as heavily shadowed or covered urban environments or underground caves, and impossible for indoor use.

All of those factors make it extremely unlikely that a GPS-based solution could be used to solve all the location identification challenges in Ad Hoc networks/Sensor networks.

\subsubsection{TDOA Location Methods}

Many other location methods are surveyed in Chapter 2. Currently, the TDOA (time difference of arrival) location method is the best one among them, however, TDOA-based methods also face some drawbacks (will be described in detail in Chapter 2), which are highlighted as follows:

- Inconsistency problem when more than two TDOA measurements are available.

- Based on triangulation only, if only two TDOA measurements are available, it is impossible to select the right result from two estimated locations. 
- Because all nodes in Ad Hoc/Sensor networks are arbitrarily distributed and moving (nodes in Sensor network may not movable), the influence of relative position between source node and reference nodes to estimation accuracy are not stable and hard to estimate (a thorough research on it is given in Chapter 3). Thus, the estimation accuracy may not be high and different estimations can not guarantee the same level of accuracy.

\subsubsection{Objectives}

In order to meet these challenges, the work reported in this thesis was started with the following objectives:

- Provide a new and practical location identification method for wireless Ad Hoc/Sensor networks

- Do not increase the hardware and software cost in this method

- Provide a location estimation method with higher accuracy than TDOA method

- Evaluate the performance of this method and compare it with TDOA method

\subsection{Contributions}

The research work reported in this thesis combines the time difference of arrival (TDOA) location identification method [7] and Cell ID [8] location estimation method and takes into account the GDOP (Geometric Dilution of Precision) theory [49-52], to 
provide a practical location identification method (called the Hybrid TDOA method) in wireless Ad Hoc/Sensor networks, with more accurate estimation than other TDOA methods. Specifically, the main contributions of this thesis include:

- A new location identification method is proposed, in which some drawbacks of the TDOA method are resolved

- The location identification system increases the accuracy of location estimation through the use of GDOP theory

- The location identification system is based only on commonly used basic assumptions in location identification research, which makes this system more practical for the real world than other TDOA improvements

- The simulation results show that the location identification system proposed in this thesis provides a more accurate estimation than other practical TDOA methods under various situations

\subsection{Thesis Organization}

The rest of this thesis is organized as follows.

Chapter 2 presents previous research work done on location identification in Ad Hoc/Sensor networks. Chapter 3 introduces the hybrid TDOA method. Chapter 4 features several simulations, verifies the hybrid TDOA method, and compares the performance of the hybrid TDOA method and another regular TDOA method in NS2. Chapter 5 gives conclusions and lays out suggestions for future work. 


\section{Chapter 2 Survey of Location Identification methods}

This survey is organized into three sections. The first section classifies the location identification technology in wireless networks from different perspectives. The second section provides an overview of major methods used in wireless location identification systems. The final section reviews past research work on the TDOA location identification method in Ad Hoc/sensor networks.

\subsection{Categories of Location Identification Technology in Wireless Networks}

There are many ways to classify location identification technology in wireless networks. In this section, several different categories are introduced and explained in detail, with examples based on various principles.

\subsubsection{Source of Measurement-based Classification}

A wireless location identification system is made up of at least two separate components: a signal detector, which performs the signal measurement, and a measuring unit, which usually has the task of calculating those signal measurements and estimating the location of the mobile node. Based on different kinds of signal, a classification is given. The radio detector in location identification systems can be $R F$ (radio frequency) based $[4,5,6]$ and can also be ultrasound based [9]. Various radio detectors have different 
pros and cons. For example, the ultrasound-based detector can provide higher accuracy but cannot tolerate even a tiny obstacle [9].

\subsubsection{Topology-based Classification}

System topology is another way to classify the different types of location identification systems in wireless networks. Table 2-1, from [6], gives an abstract classification that is based on differences between system topologies.

Table 2-1 Classification based on topology

\section{Classification of Location Identification Systems}

\begin{tabular}{|l|l|}
\hline Name & Description \\
\hline Remote Identification of Location & Location estimation is done from remote site \\
\hline Self-Identification of Location & $\begin{array}{l}\text { Location measurement is done from mobile } \\
\text { unit, usually based on other reference nodes }\end{array}$ \\
\hline Indirect Remote Identification & $\begin{array}{l}\text { Self-identification system plus data transfer of } \\
\text { measurement result to remote site }\end{array}$ \\
\hline Indirect Self-Identification & $\begin{array}{l}\text { Remote identification system plus data transfer } \\
\text { of measurement result to mobile unit }\end{array}$ \\
\hline
\end{tabular}

\section{Remote identification systems}

This system collects a mobile node's signals and determines its location in a centralized server. The main advantage of remote identification is that the mobile nodes that need to identify their geographical locations can be small, cheap, and power efficient. On the other hand, such system costs a complex system and backbone networks used to transfer necessary information between different nodes [6].

\section{Self-identification systems}


In the self-identification system, a mobile node that needs to know its position makes the appropriate signal measurements from other reference nodes and uses these measurements to estimate its geographic location. The most popular form of self-identification system used is GPS [5].

\section{Indirect location identification systems}

The position measurements from the self-positioning receiver can be sent to a remote site or vice versa through a data link. A self-positioning system that sends position data to a remote location is referred to as indirect remote positioning, and a remote positioning system transmitting an object's position to the object is referred to as indirect self-positioning.

All above four systems have different system topologies, but they can share the same measurement method.

\subsubsection{Measurement Technology-based Classification}

Many kinds of measurement technologies are used in location identification systems, such as signal strength, signal pattern matching, direction of arrival, time of arrival, time difference of arrival, etc. Some of the representative and practical technologies will be introduced in this section.

\section{Signal strength}

Radio propagation has an important characteristic in that the radio signal increases attenuation as the distance between the receiver and signal source increases. Extensive 
research has been done on the propagation of radio frequency $(R F)$ in different environments. Many models have been built for various scenarios [1].

In [10], the authors suggest locating the position of mobile nodes based on $R F$ signal strength in indoor environments. They employed a wall attenuation factor (WAF)-based $R F$ signal propagation model to estimate the distance from target $R F$ signal. Then, by using more than one measurement, the mobile node's position can be located. This system in [10], called the RADAR system, cannot obtain accuracies higher than the $R F$ mapping of signal strengths corresponding to various locations for their system [11].

In [11], the authors propose another location identification method based on a relatively easy $R F$ propagation model. Compared with [10], the approach in [11] obtains higher accuracy in outdoor environments and requires less infrastructure support. However, the method in [10] yields highly accurate results in an indoor environment, as opposed to [11]. The problem of methods based on signal strength is that they are highly related to the $R F$ radio propagation models. Different models can even lead to opposite results [12]. Recently, however, because of the absence of an $R F$ radio propagation model that is sophisticated enough to describe all the features of signal propagation in nature, all those methods yield different drawbacks in different environments. In [13], the authors prove that the accuracy of signal strength in general cannot compare with the angle of arrival methods that will be described later.

\section{Signal pattern matching}

Instead of detecting signal strength or signal timing, the signal pattern matching 
method relies on the structural characteristics of the $R F$ signal. This method employs the multi-path phenomenon of the $R F$ signal. The signal pattern matching method designs a unique signature for each given location by combining the multi-path pattern with other signal characteristics.

The U.S. Wireless Corporation [14] has implemented a representative commercial system called the RadioCamera system. In this system, a signal signature database of the location grid of a specific service area is kept. This system requires a pre-run process before the wireless network is deployed. In the pre-run process, the database is generated. In the RadioCamera system, typically, a vehicle drives through the coverage area transmitting signals to a monitoring site. Then the system analyzes the incoming signals, compiles a unique signature for each square in the location grid, and stores it in the database. In order to locate a certain mobile node, the RadioCamera system matches the mobile unit's signal signature to an entry in the database [14]. The major problem with this method, like RADAR [10], is the substantial effort needed to generate the signal signature database beforehand [11]. Hence, this method is not suitable for most Ad Hoc/Sensor network scenarios, in which a pre-run process is impossible.

\section{Direction of Arrival (DOA)}

As mentioned in [13], there is another method for estimating the location of the mobile node, which is to detect the angle of each reference node with respect to the source node in a certain reference system. Then, by employing triangulation, the location of a mobile node can be identified. 
Triangulation is the process of finding coordinates and distance to a point by calculating the length of one side of a triangle. In triangulation, the location of the source node can be identified by given measurements of the angles and sides of the triangle formed by that point and two other known reference points, using the law of sines. The following location methods, including DOA, AOA (Angle of Arrival), TOA (Time of Arrival), and TDOA, are all developed from triangulation.

This method is called Direction of Arrival (DOA) or Angle of Arrival (AOA). In detail, the AOA method employs multi-array antennas to estimate the direction of arrival of the signal from the source node. In that way, a measurement can restrict a line along which the source node should be. If two such measurements are available from two antennas at two different locations, the location of the signal source can be identified at the intersection of the lines of bearing from the two antennas. Moreover, estimation accuracy can be increased by employing multiple DOA measurements using the redundant information.

Many studies have been done on AOA/DOA methods in wireless systems $[15,16,17]$. A typical example of AOA/DOA system is the VOR/VORTAC stations, discussed in [15]. This system was used for long-distance aviation navigation prior to GPS [15]. In this system, a so-called VOR station transmits a unique omni-direction signal that allows an aircraft to determine its bearing relative to the VOR station. The VOR signal is electrically phased, making the signal different through the different parts of a $360^{\circ}$ circle. The aircraft can estimate the direction of VOR stations relative to its current position by 
detecting which radials of the $360^{\circ}$ different parts are received.

Although the AOA/DOA method is a practical solution for mobile node location identification in wireless networks, there are still some inherent drawbacks to this method. First, to employ such method, extra equipment associated with the antenna arrays is needed, which increases the cost on the deployment of a wireless network considerably. Second, a critical requirement of AOA/DOA estimation is that the antenna arrays should come from the Line-of-Sight (LOS) direction. However, this is not the case in some scenarios, such as heavily shadowed channels, e.g. those encountered in urban or indoor environments. The last problem with the AOA/DOA method is that the AOA/DOA algorithms are too complex to implement due to the need for measurement, storage, and usage of array calibration data and their computationally intensive nature [18].

\section{Round-trip Time of Flight}

The Round-trip Time of Flight (RTOF) approach does not require absolute time synchronization. In the RTOF method, a more moderate relative clock synchronization system replaces the requirement of an absolute synchronization system. For a RTOF measurement, the measuring unit more or less acts as a common radar system. A measuring unit responds to the interrogating radar signal, and then the complete round-trip propagation time is measured. Based on the round-trip times, the location of a mobile node can be identified using triangulation. However, in the RTOF method, the exact delay/processing time caused by the responder should be measured accurately, which is very difficult to meet [6], and a relative clock synchronization system in all 
nodes in the network is also hard to maintain.

\section{Time of Arrival}

This is a method for estimating the location of the source node based on indirectly determining the time that the signal takes from the source node to the reference node on the forward or the reverse link. It estimates the source node's location by measuring the time, in which the source node responds to an inquiry from some other mobile nodes (reference nodes) whose locations are known. The total time elapsed from the moment that the command is transmitted to the instant when the mobile source node's response is detected. The total time is composed of the sum of the round trip signal delay and any processing and response delay within the source node. Assuming that the processing delay of the desired response in the source node is measured accurately, the total round trip delay can be ascertained by subtracting the processing delay. In that way, the approximate distance of the mobile from the source and the receiver can be estimated. If the response of a source node can be detected by two extra receivers (reference nodes) that know their locations, then the location of the source node can be located using the triangulation method.

This method also faces some nature-born problems. First, it is difficult to estimate the response delay within a mobile node in practice, because there are design and manufacturing variations between different mobile nodes. Second, the timing errors in the absence of Line-of-Sight (LOS) can heavily influence the accuracy of TOA. Moreover, there is no way to reduce the errors because of the multiple signal reflections on the 
forward or reverse link. Furthermore, to measure the time, the whole network needs an absolute timing synchronization, which requires extra, expensive equipment, greatly increasing the cost of the whole network, and requires high resource consumption to maintain.

\section{Time Difference of Arrival}

An improvement was made to the TOA method, the so-called Time Difference of Arrival (TDOA) method. The TDOA method depends on estimating the difference in the arrival times of the signal from the source node at multiple receivers (reference node). The TDOA method takes a snapshot of the signal at a synchronized time period at reference nodes. The cross-correlation of the two types of signal at the pairs of reference nodes is done and the peak of the cross-correlation output gives the time difference for the signal arrival at those two reference nodes. Assuming that the source node and reference nodes are coplanar, a particular algorithm for estimating the value of the time difference can be used to define a hyperbola between the two reference nodes on which the source node may exist. If this procedure is done again with another reference node in combination with any of the reference nodes used previously, another hyperbola is defined and the intersection of the two hyperbolas results in the location identification of the source node. This method is also sometimes called a hyperbolic location identification method.

The TDOA method provides several advantages over the other methods described above. First, the required changes in order to incorporate the TDOA method are only 
limited to the software of the system, no hardware modification is necessary on the mobile nodes. In this respect, it is more cost effective than a GPS-based solution, and this method will not decrease the life time of mobile nodes, because it does not need the battery of a mobile node to supply extra equipment. Of course, computation consumes power also, but it will not cost much. Moreover, TDOA does not require an absolute timing system of the transmission, compared with the TOA method requirements, which significantly decreases the software design and relative protocol design issues. In addition, the TDOA method can work accurately in some scenarios where there is no LOS signal component. Overall, the TDOA method is superior to the other methods described above.

\section{Cell-based Method}

In wireless cellular systems such as GSM and CDMA, base stations separate the space into a hexagonal structure, as shown in Figure 2-1.

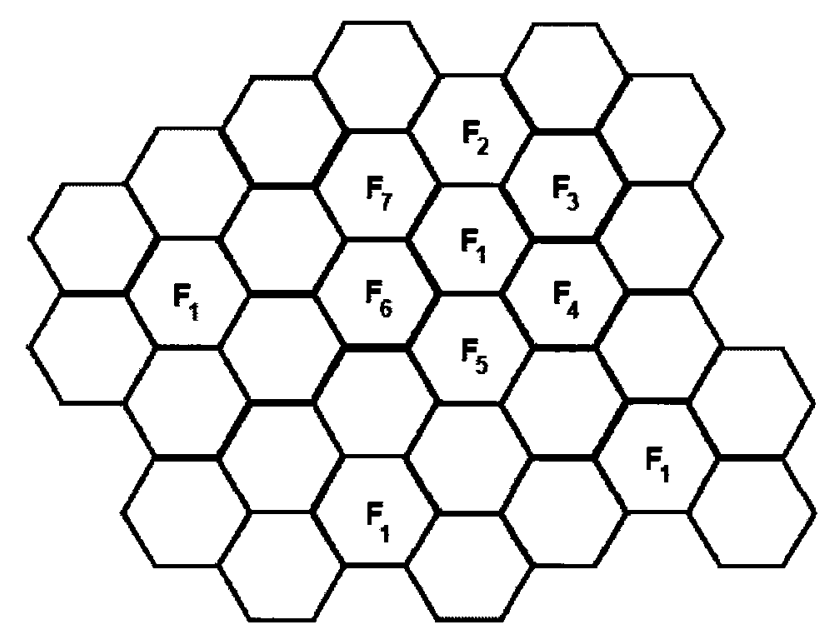

Figure 2-1 Cells in cellular wireless system

In Ad Hoc/Sensor networks, a similar scenario exists. If those reference nodes are 
treated as "base stations," then the communication ranges of those reference nodes could be treated as "cells" also. The following figure (Figure 2-2) shows the situation in Ad Hoc/Sensor networks, where " $P_{i}$ " refers to a reference node.

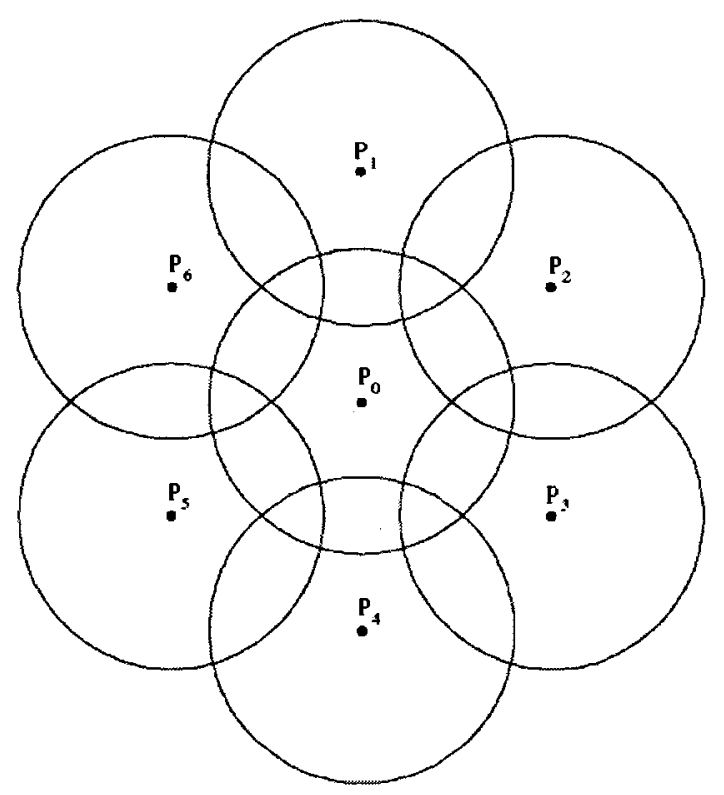

Figure 2-2 Cells in Ad Hoc/Sensor networks

The cell-based location identification system simply utilizes the characteristic of cells overlapping in geometry $[8,19]$. A cell-based location identification system can be described as follows: Consider any physical layout of the mobile nodes in the wireless system; i.e., Figure 2-2 shows a hexagonal structure distribution of the reference nodes. The area covered by a reference node could be noted as a "cell." Ideally, each cell has a circular shape, as shown in Figure 2-2. If a mobile node that is arbitrarily distributed among the wireless network is within a cell belonging to a certain reference node, the mobile node can receive the $R F$ signal containing the cell number and reference location information from that reference node. Additionally, in Ad Hoc/Sensor networks, a mobile 
node may receive the $R F$ signals of more than one reference node, if that mobile node is in a signal-overlapping area (the blue-shadowed area), as shown in Figure 2-3:

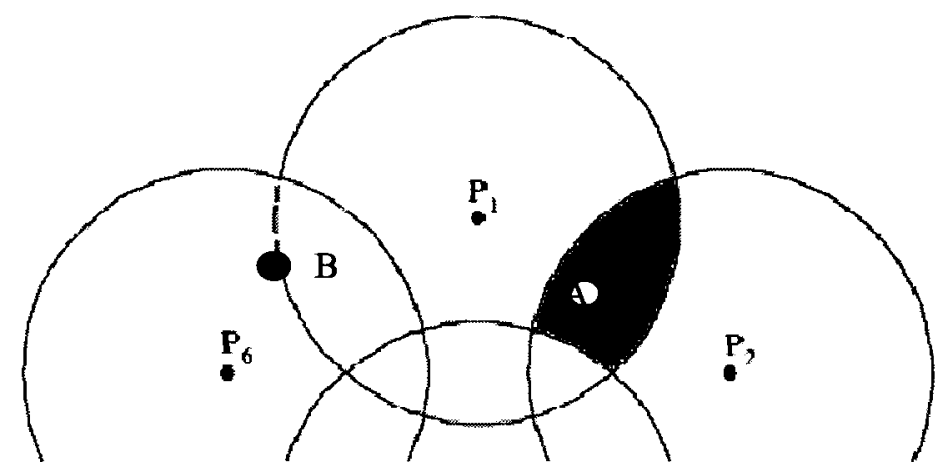

Figure 2-3 Overlapping area between cells

A mobile node in area A can only receive the signals from reference nodes $P_{l}$ and $P_{2}$, and a mobile node in area $\mathrm{B}$ can receive the $R F$ signals from reference nodes $P_{1}$ and $P_{6}$. Thus, the probable location of a mobile node can be determined by the $R F$ signals received from the neighbor reference nodes. The two-dimensional space can be defined as an area where the mobile nodes can receive a unique set of $R F$ signals from the reference nodes. Thus, Figure 2-2 can be assigned into different areas, as follows: 


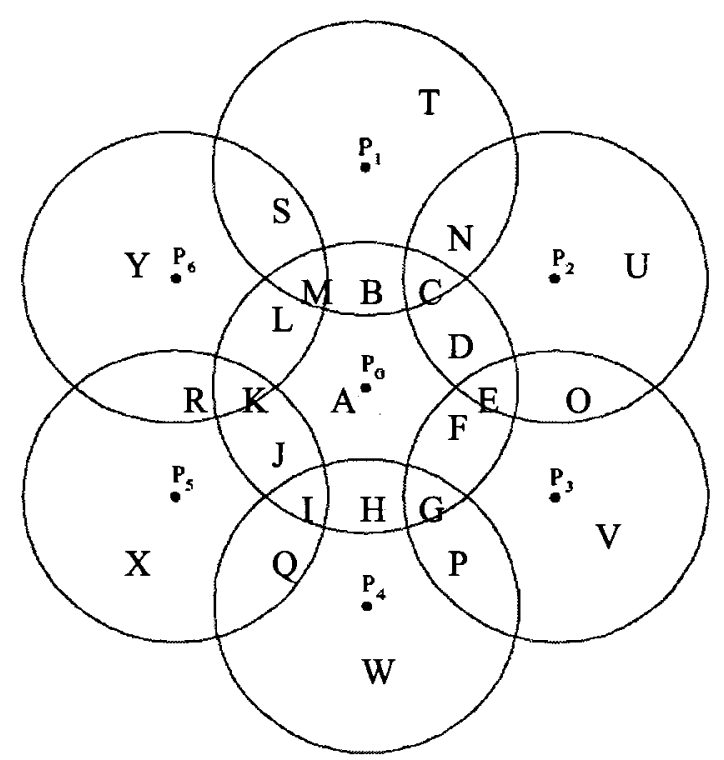

Figure 2-4 Cell area classification in Ad Hoc/Sensor network

In Figure 2-4, there are A to Y 25 unique areas. A mobile node can identify itself in an area by receiving the $R F$ signals of those reference nodes. In this way, the mobile node's location can be identified.

It is clear that cell-based location identification cannot provide estimations as highly accurate as the other methods described above. However, the cell-based method is the simplest and costs less than all the other methods described in this chapter.

\subsubsection{Overview of Current Wireless Location Identification Systems}

Currently, most of the available solutions are based on the TDOA method. The most important benefit of the TDOA method is that only the measuring units (reference nodes) must be synchronized, which can be done by a backbone network or any kind of reference transponder in a known position [6], and the measurement error of the TDOA 
method is inversely proportional to the bandwidth, as well as independent of the distance between the source node and the reference node [20]. Moreover, almost all time-based location identification systems employ signals and signal evaluation concepts that are well known, from modern radar systems [6], and TDOA provides better noise tolerance than other methods [6].

The authors of [6] drew a figure (Figure 2-5) depicting a rough overview of current wireless location systems. The TDOA and RTOF systems based on OFDM (Orthogonal frequency-division multiplexing) (IEEE802.11 physical layer) can provide best accurate as it shown. However, in Cellular systems, the accuracy is not as good as WLAN networks based on OFDM.

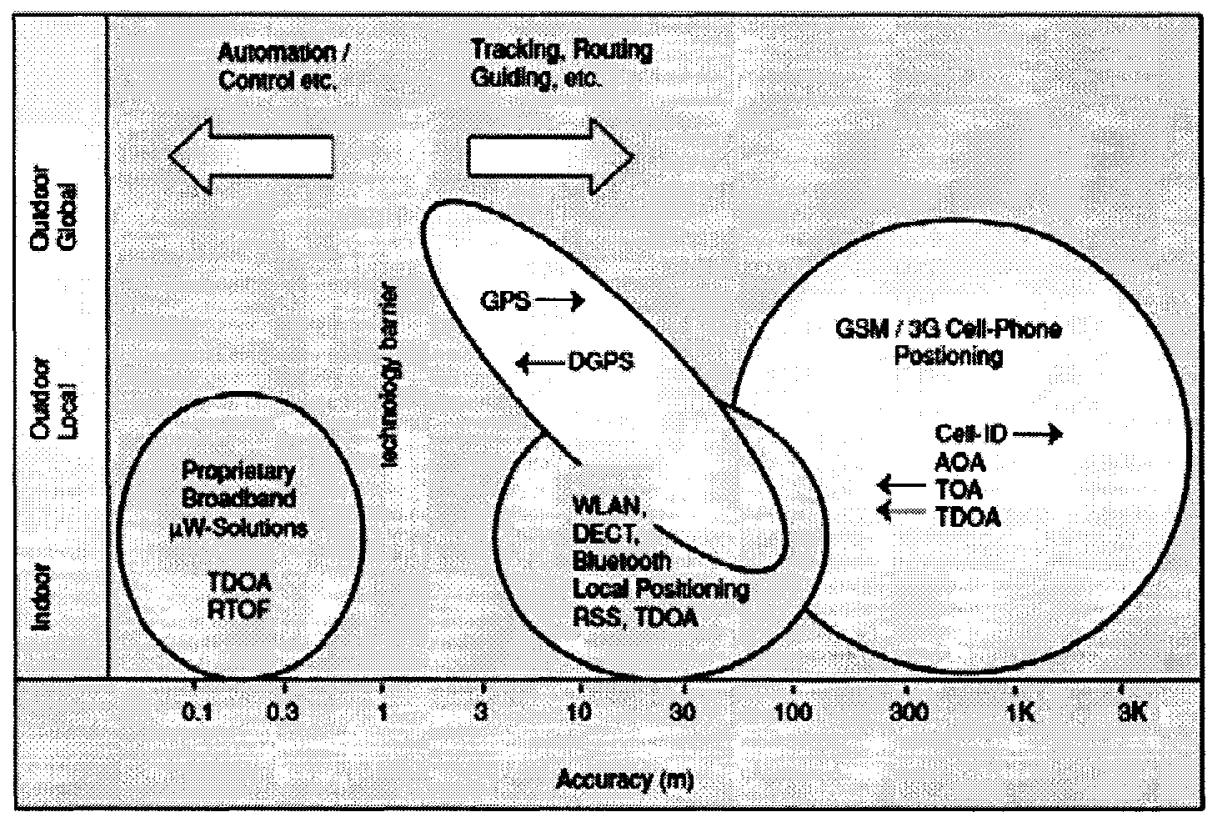

Figure 2-5 Overview of wireless location systems [6]

Another figure (Figure 2-6) is given in [20] to reflect the positioning accuracy and the $R F$ bandwidth employed in wireless technology by a certain type of wireless network. As 
Figure 2-6 shows that the RF bandwidth also influences the accuracy of location identification systems. Basically, the IEEE 802.11 series provide good physical conditions for positioning, which are the most popular physical implementations in $\mathrm{Ad}$ Hoc/Sensor networks.

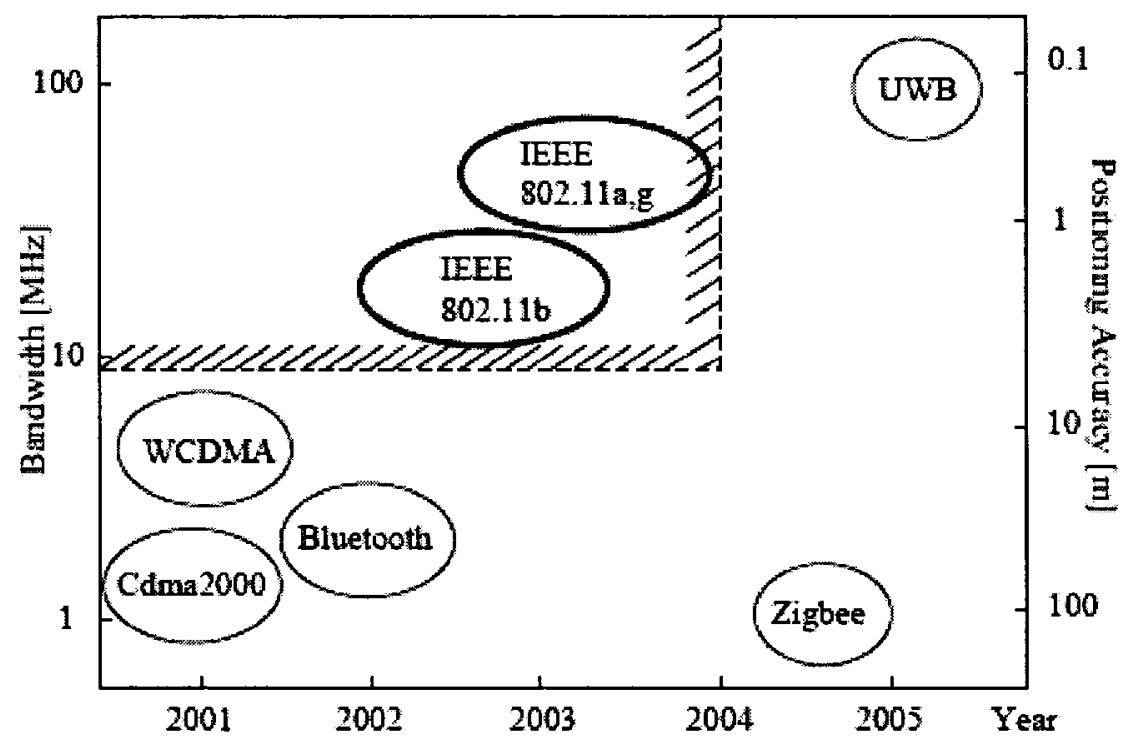

Figure 2-6 Comparison between estimation accuracy and wireless systems [26]

A table (Table 2-2) from [20] also shows a performance comparison of those methods.

Table 2-2 Performance comparison of location identification systems

\begin{tabular}{|l|l|l|l|}
\hline \multirow{2}{*}{ Method } & \multicolumn{2}{|l|}{ Accuracy Degradation } & Complexity \\
\cline { 2 - 3 } & Multipath & $\begin{array}{l}\text { Distance } \\
\text { of AP-STA) }\end{array}$ & \\
\hline TOA(TDOA) & Slightly & None & Medium \\
\hline AOA & Slightly & Slightly & High \\
\hline RSSI & Severely & Slightly & Low \\
\hline Cell-ID & None & Severely & Low \\
\hline
\end{tabular}


From above descriptions, it is clear that the TDOA method can provide relatively better performance, with relatively acceptable lower costs among those methods.

The method that provides the most practical alternative to the TDOA method is probably the AOA method, discussed above. In fact, it was proven that in cellular wireless systems, the most accurate location identification system is a hybrid TDOA/AOA method $[40,41,42]$. The AOA techniques face certain problems. To deploy AOA systems, the additional cost of adaptive antenna arrays on all mobile nodes is unavoidable (unfortunately, the cost of such antenna arrays is even higher compared with GPS receivers), and AOA is also vulnerable in the absence of a LOS path between the source node and the reference nodes.

On the other hand, the only precondition for the TDOA measurement is a precisely synchronized clock at those reference nodes. In this thesis, we assume that those reference nodes have GPS receivers, in which the correspondence to the timing standards is already provided. This requirement is only for those reference nodes, not to other mobile nodes in the network.

\subsection{TDOA Location Identification in Wireless Networks}

TDOA location identification systems have been developed for a fairly long time in wireless networks $[4,6$ 7]. TDOA location identification estimation is accomplished in two steps. First, it involves measurements of the time differences of the signals that arrive from a source, between pairs of reference nodes through the use of time delay estimation 
techniques. This is referred to as TDOA measurement in this thesis. Second, the TDOA measurements obtained are transformed into range differences between reference nodes, resulting in a set of nonlinear hyperbolic equations [21]. In the second stage, an efficient algorithm should be provided in order to produce an unambiguous solution to the nonlinear equations, and then the result from the calculation from the algorithm gives the estimated location of the source mobile node.

\subsubsection{TDOA Location Estimation}

The time difference of a signal from a source node to different reference nodes can be estimated using cross-correlation techniques, in which the signal received at one reference node is correlated with the signal received at another reference node [21]. The timing requirement for this method is the synchronization of all the reference nodes participating in the TDOA measurements. A significant amount of research on cross-correlation techniques in the $R F$ area has been done, and this thesis will not focus on how the cross-correlation techniques work. Interested readers can refer to [21- 29]. Cross-correlation techniques have been shown to be effective in reducing the effects of noise and interference [24].

After the time difference measurements have been obtained, those measurements can be converted into nonlinear hyperbolic equations. Using triangulation theory, after solving those equations, the location can be identified. However, as those equations are non-linear, solving them is not a trivial operation. Many algorithms have been proposed 
for this purpose, with different complexities and accuracies.

If, in a two-dimensional space, two TDOA measurements can be obtained from three reference nodes, a consistent system can be deduced, in which two equations obtained from the two TDOA measurements are used to solve for two unknowns. However, if more than three reference nodes have obtained the signal of the source node (assume, $M$, $M>3$ ), any three reference nodes from $M$ reference nodes can result in a estimated location, which makes the system inconsistent, because the solution of the source node's location is not unique.

Furthermore, if there are only three reference nodes, the non-linear equations will result in two sets of locations of source nodes. It is obvious in triangulation. However, only one result is correct.

If $M$ reference nodes can get the $R F$ signal from the source node, there are $C_{3}^{M}$ combinations of three reference nodes; thus, $C_{3}^{M}$ results of the source node's location can be obtained. In [30-32], some assumptions and some associated technology are used to put different weights on different combinations. Thus, the final result is derived from the average value of those combinations, based on their weights. But without those assumptions and associated technology, a regular TDOA takes the average value of all combinations as the final result.

\subsubsection{Numerical Analysis and Least Square Method}

Numerical analysis is the study of algorithms for continuous mathematical problems. 
It focuses on practical mathematical calculations. Numerical analysis does not seek exact answers, because, in practice, exact answers are impossible to obtain for those problems. Instead, numerical analysis is concerned with obtaining approximate solutions while maintaining reasonable bounds on errors. The Least Square Method is one of the most important methods in numerical analysis. It is used to model numerical data obtained from observations by adjusting the parameters of a model so as to get an optimal fit of the data, in which the best fit is characterized by the sum of squared residuals have its lowest value. In the TDOA method, the least square is commonly used to optimize the estimation of a source node's location when $M$ is bigger than three.

If reference nodes are arbitrarily distributed throughout the wireless network, which is the typical scenario in Ad Hoc/Sensor networks, location identification becomes very complex. Friedlander, in [31, 33], utilized a least square (LS) and a weighted LS error criterion to solve the inconsistency problem and produce the location of the source node. However, the weight is still difficult to obtain [33]. The authors of [32] proposed a divide and conquer $(\mathrm{DaC})$ method. They suggested dividing the TDOA measurements into groups, each with a size equal to the number of unknowns. The solution of the unknowns is calculated for each group, and then appropriately combined to provide a final solution. This method requires an equal number of range difference measurements in each group, and as a result, the TDOA estimates from the remaining sensors cannot be used to improve accuracy. In $[34,35]$, the authors assumed that the initial value of the source node's location is known, so the correct position of the source node can be obtained in the 
subsequent measurements.

In conclusion, all those proposed algorithms are trying to solve the inconsistency problem and the problem of how to pick the correct result if there are only three reference nodes. In above research, by using the least square method, the weighted least square method, weights on different TDOA measurements, grouping the reference nodes, extra assumptions on mobile nodes, and so on, the two problems could be solved, however those assumptions and preconditions in above papers are hard to obtain or hard to satisfy (like the assumptions in $[34,35]$ ). A solution that can solve the two problems and provide high accurate location estimation, without needing extra tough conditions, is obviously welcomed.

A detailed discussion of the two problems will take place in the next chapter.

\subsubsection{Improved TDOA in Wireless Ad Hoc/Sensor Networks}

Although until now, the TDOA has been the most popular location identification method in wireless networks worldwide, it still suffers from some of the problems described in the previous sections. In order to increase performance and solve those drawbacks of the TDOA method, many papers have been published.

Linghan Fei and Jinrong Yin in [35] fundamentally showed the correlation between location precision and radar (reference node) arrangement. They tried to use reference nodes in arranged positions to increase the performance of the TDOA method. This is a good idea for wireless systems with fixed infrastructures, like GSM and CDMA, but 
impossible for Ad Hoc/Sensor networks, in which the nodes are distributed arbitrarily and move randomly.

In [35], the inconsistency problem is not a big issue, because four, and only four, reference nodes are employed in the location identification. By arranging the positions of the reference nodes, the inconsistency problem is solved at a certain level. However, this problem still exists in those Ad Hoc/Sensor networks where it is not known how many reference nodes are around a source node in a certain location identification process.

Others try to utilize additional algorithms or equipment to improve the performance of the TDOA method. The authors in [36] introduced an integration method involving Global Positioning system (GPS) pseudo-ranges and TDOA measurements from the pilot signal of a wireless communication network (a cellular network) to solve the navigation problem in case where the number of visible GPS satellites is not sufficient. In essence, this paper employed the GPS system to provide a reference system so that the right location result can be identified from the two calculated results.

$[37,38]$ proposed an original solution called enhanced TDOA, based on Ultra Wide Band (UWB) technology, using millimeter multi-tone dual transmission, which acts like a pulse composite signal and a basic millimeter receiver as the reference node. A sketch is shown in the figure below. 


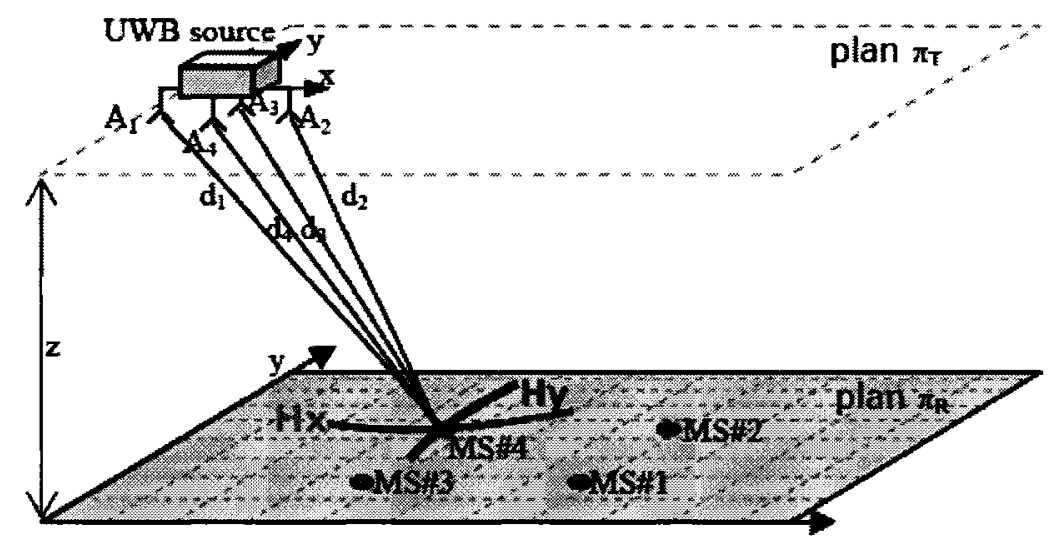

Figure 2-7 Sketch of mobile stations (source nodes) localization

The Ultra Wide Band technology is a natural approach to mitigate multi-path and allow accurate time measurement. UWB technology is currently a very popular research subject worldwide. By employing such technology, an Ad Hoc/Sensor network can be efficiently managed in terms of connectivity and energy efficiency [39]. In [37, 38], the UWB source behaves like a "God" in the Ad Hoc network: it takes responsibility for all the reference nodes. Figure 27 shows that the UWB source can be reached by all mobile nodes and has four receivers in different positions, behaving like four reference nodes always in the way. This is a good solution, but unfortunately, most Ad Hoc/Sensor network scenarios cannot provide a "God" node, like the UWB source. Moreover, this kind of "God" node also makes such Ad Hoc/Sensor network vulnerable. For example, if, in an Ad Hoc network for soldiers on a battlefield, the UWB source is destroyed, the location identification functionality as a whole goes down.

In a nutshell, many improved TDOA algorithms have been proposed. However, no one has been able to produce a practical location identification method with high 
accuracy in a simple, cheap Ad Hoc/Sensor network using simple wireless MAC protocols, such as the IEEE 802.11 series, without employing specific powerful nodes in the network, and without deploying specific equipment to support this functionality. 


\section{Chapter 3 Hybrid TDOA Method}

This chapter first describes the assumptions that this thesis has adopted. Second, thorough research on the TDOA method is provided, in which a solution to nonlinear equations based on the estimated time difference is proposed. Third, the Cell ID location identification method is described. Following that, the GDOP (Geometric Dilution of Precision) theory is introduced. Finally, the original Hybrid TDOA algorithm is proposed.

\subsection{Research Assumptions}

In this thesis, we focus on proposing a practical location identification system for Ad Hoc/Sensor networks without additional specific requirements and assumptions. Thus, the following assumptions are held in this thesis.

1. An Ad Hoc/Sensor network without a super node or master node that can provide much higher computing capability or larger radio coverage area than other nodes.

2. Some nodes in our Ad Hoc/Sensor networks can synchronize themselves and locate their positions by GPS or the support of some other system; these nodes are called reference nodes. Other nodes that have no such ability are called source nodes.

3. We focus on small nodes that have short-range radio frequency $(R F)$ transceivers 
(in the simulation implementation, the IEEE802.11 series protocol is employed for the physical layer). The primary goal is to leverage this radio for localization.

4. In our Ad Hoc/Sensor networks, preplanning or extensive infrastructure is impossible.

5. In our Ad Hoc/Sensor networks, all nodes can be arbitrarily distributed and freely move (nodes in some kinds of Sensor networks may not movable). Moreover, those nodes may have modest processing capabilities and limited energy resources.

\subsection{TDOA Location Identification Method}

This section provides a deep study of the TDOA technique of location identification. Moreover, the different algorithms that are used to solve the resulting hyperbolic equations, using TDOA measurements, are discussed.

\subsubsection{TDOA Measurement}

TDOA techniques use time differences, as opposed to GPS, which uses absolute time measurements. The time difference is expressed as:

$$
\Delta t_{i}=t_{i}-t_{0}
$$

where $t_{0}$ is an unknown parameter and equals the absolute arrival time at the original reference node, $t_{i}$ is the unknown absolute arrival time at $i^{\text {th }}$ reference node; in Equation $1\left(1\right.$, only the time difference $\Delta t_{i}$ is known. Assuming propagation in a two-dimensional 
(2-D) isotropic medium (the 3-D situation is similar), the equation governing reception of the $R F$ signal at the $i^{\text {th }}$ reference node can be given as [31]:

$$
\left(x-x_{i}\right)^{2}+\left(y-y_{i}\right)^{2}=c^{2}\left(\Delta t_{i}+t_{0}\right)^{2}
$$

where $c$ is the speed of propagation, $(x, y)$ is the location of the mobile source node. $\left(x_{i}, y_{i}\right)$ is the location of the $i^{\text {th }}$ reference node.

The TDOA of a $R F$ signal $\left(\Delta t_{i}\right)$ can be estimated using two general methods [21]. First, a relative TDOA measurement can be produced by subtracting the relative TOA measurements from two reference nodes. Second, using cross-correlation techniques, the received signal at one reference node is correlated with the received signal at another reference node. An introduction to cross-correlation techniques can be found in Appendix B.

For the first method, an absolute TOA measurement is not necessary either, but a timing synchronization between the reference nodes is necessary, so the TDOA measurements can be derived by subtracting the times when those reference nodes receive the source node's signal. This method is simple, but vulnerable to noise and interference.

The timing requirement for the cross-correlation method is the synchronization of all the reference nodes in the TDOA measurements as well, which is practical in our scenario. 


\subsubsection{Nonlinear Equations Calculation}

After the TDOA measurements have been obtained, the data can be converted into nonlinear hyperbolic equations. Many algorithms with different complexities are proposed for this purpose. This section introduces the mathematical model used to solve those non-linear equations $[31,43,44]$, and our solution to the non-linear equations is proposed.

The general model for 2-D location identification of a source mobile node using $K$ reference nodes can be developed. A series of index $i=1,2,3, . k-1$, assigned to those reference nodes. The index " 0 " is assigned to the reference node that first received the $R F$ signal of the source node. Clearly, the $0^{\text {th }}$ reference node is the node that is nearest to the source node. Assuming $(x, y)$ is the location of the source node and $\left(x_{i}, y_{i}\right)$ is the location of the $i^{\text {th }}$ reference node, the following equation of the range distance $\left(R_{i}\right)$ between the source node and the $i^{\text {th }}$ reference node can be obtained easily.

$$
\begin{aligned}
& R_{i}=\sqrt{\left(x_{i}-x\right)^{2}+\left(y_{i}-y\right)^{2}} \\
& =\sqrt{x_{i}^{2}+y_{i}^{2}-2 x_{i} x-2 y_{i} y+x^{2}+y^{2}}
\end{aligned}
$$

Then, based on the TDOA measurements, the range difference between other reference nodes to the $0^{\text {th }}$ reference node can be given by:

$$
\begin{aligned}
& R_{i, 0}=c \Delta t_{i}=R_{i}-R_{0} \\
& =\sqrt{\left(x_{i}-x\right)^{2}+\left(y_{i}-y\right)^{2}}-\sqrt{\left(x_{0}-x\right)^{2}+\left(y_{0}-y\right)^{2}}
\end{aligned}
$$

where $i$ is the index of a reference node, $R_{i, 0}$ is the range difference between the range from the source node to the $0^{\text {th }}$ reference node and the range from the source node to the 
$i^{\text {th }}$ reference node, $R_{0}$ is the distance between the $0^{\text {th }}$ reference node and the source node, and $\Delta t_{i}$ is the TDOA measurement of the time difference between the $0^{\text {th }}$ reference node and the $i^{\text {th }}$ reference node. Equation 4 gives a series of equations to the $K-1$ reference node. The solution of the former set of nonlinear hyperbolic equations can produce the two-dimensional coordinates of the source node.

It is clear that solving the set of equations in Equation 4 is not easy. Consequently, it is necessary to linearize Equation 4 . The commonly used mathematical way to do this is to use the Taylor-series expansion, retaining the first two terms [43]. But another alternative that can reduce the complexity of the Taylor-series expansion method is employed by most real systems [31, 44]. First, the set of nonlinear equations (Equation 4) are transformed into another form, as follows:

$$
R_{i}^{2}=\left(R_{i, 0}+R_{0}\right)^{2}
$$

Then Equation 3 can be rewritten as follows:

$$
R_{i, 0}^{2}+2 R_{i, 0} R_{0}+R_{0}^{2}=x_{i}^{2}+y_{i}^{2}-2 x_{i} x-2 y_{i} y+x^{2}+y^{2}
$$

Thus, by subtracting Equation 3 from Equation 5, where $i$ equals 0 , the following equation is obtained:

$$
\begin{gathered}
R_{i, 0}{ }^{2}+2 R_{i, 0} R_{0}+R_{0}{ }^{2}-R_{0}{ }^{2}=x_{i}{ }^{2}+y_{i}{ }^{2}-2 x_{i} x-2 y_{i} y+x^{2}+y^{2}-\left(x_{0}{ }^{2}+y_{0}{ }^{2}-2 x_{0} x-2 y_{0} y+x^{2}+y^{2}\right) \\
=x_{i}{ }^{2}+y_{i}{ }^{2}-2\left(x_{i}-y_{0}\right) x-2\left(y_{i}-y_{0}\right) y-x_{0}{ }^{2}-y_{0}{ }^{2}
\end{gathered}
$$

Then, the following result is obtained:

$$
R_{i, 0}{ }^{2}+2 R_{i, 0} R_{0}=x_{i}{ }^{2}+y_{i}{ }^{2}-2 x_{i, 0} x-2 y_{i, 0} y-x_{0}{ }^{2}-y_{0}{ }^{2}
$$




$$
\begin{aligned}
x_{i, 0} & =\left|x_{i}-x_{0}\right| \\
\text { where } y_{i, 0} & =\left|y_{i}-y_{0}\right|
\end{aligned}
$$

With different $i$ (reference node Id), a set of equation 6 can be obtained. The set of Equation 6 are now linear with the source node $(x, y)$. If the number of nonlinear hyperbolic equations equals the number of unknown coordinates, then a unique and consistent solution can be determined. For example, in two-dimensional space, in order to determine the location of a mobile source node, three reference nodes are enough to provide two TDOA measurements $\left(1^{\text {st }}\right.$ and $2^{\text {nd }}$ relative to reference node $\left.0^{\text {th }}\right)$.

Additionally, the topologies of the reference nodes should be considered. There are two kinds of algorithms. One is for structurally placed or linearly placed reference nodes, such as in most cellular systems (GSM and CDMA), in which the Base Transceiver Stations (BTS) that are also used as reference nodes in location identification services are organized in specific positions throughout the network. The second type is for arbitrarily placed reference nodes. The first type is not suitable for the scenarios described in this thesis. Interested readers can refer to $[28,29]$.

In this thesis, only the second kind of algorithm is considered. In such scenario, the reference nodes are arbitrarily distributed and freely move, which makes the localization algorithm complex. Other than the complexity, another issue is also important: how to keep the consistency of the system of estimated locations, when there are more than three reference nodes that provides more than two TDOA measurements. Moreover, in Ad Hoc/Sensor networks, this may happen with higher probability than in other wireless 
networks, because the nodes in Ad Hoc/Sensor networks are moving or arbitrarily placed. In hybrid TDOA method, a new way of solving the inconsistency problem is proposed by employing GDOP theory, in which the inconsistency problem is solved and also obtains higher accurate estimations.

In two-dimensional space, three reference nodes are enough to locate a source node. A coordinate system can be built to ease the computation by assuming the $0^{\text {th }}$ reference node is located at $(0,0)$, the $1^{\text {st }}$ reference node is located at $\left(x_{l}, 0\right)$, and the $2^{\text {nd }}$ reference node is located at $\left(x_{2}, y_{2}\right)$ [45]. Thus, the following development can be easily produced:

$$
\begin{aligned}
& R_{0}=\sqrt{\left(x_{0}-x\right)^{2}+\left(y_{0}-y\right)^{2}}=\sqrt{x^{2}+y^{2}} \\
& x_{i, 0}=x_{i}-x_{0}=x_{i} \\
& y_{i, 0}=y_{i}-y_{0}=y_{i}
\end{aligned}
$$

And, in the new coordinate system, Equation 6 can be rearranged as:

$$
\begin{aligned}
& 2 R_{1,0} R_{0}=x_{1}{ }^{2}-2 x_{1,0} x-R_{1,0}{ }^{2} \\
& 2 R_{2,0} R_{0}=\left(x_{2}{ }^{2}+y_{2}{ }^{2}\right)-2 x_{2,0} x-2 y_{2,0} y-R_{2,0}{ }^{2}
\end{aligned}
$$

Then, by using the two equations in Equation 8, the linear function of the coordinates $(x, y)$ is obtained as follows:

$$
\begin{aligned}
& y=a x+b \\
& a=\frac{R_{2,0}\left(x_{1} / R_{1,0}\right)-x_{2}}{y_{2}} \\
& b=\frac{x_{2}{ }^{2}+y_{2}{ }^{2}-R_{2,0}{ }^{2}+R_{2,0} R_{1,0}\left(1-\left(x_{1} / R_{1,0}\right)^{2}\right)}{2 y_{2}}
\end{aligned}
$$

By substituting the first linear equation of Equation 9 from the first equation of Equation 8, a function about $x$ can be obtained, as follows: 


$$
\begin{aligned}
& c x^{2}+d x+e=0 \\
& \text { where } \\
& c=\left(\frac{x_{1}}{R_{1,0}}\right)^{2}-1-a^{2} \\
& d=x_{1}\left[1-\left(\frac{x_{1}}{R_{1,0}}\right)^{2}\right]-2 a b \\
& e=\frac{R_{1,0}{ }^{2}}{4}\left(1-\left(\frac{x_{1}}{R_{1,0}}\right)^{2}\right)^{2}-b^{2}
\end{aligned}
$$

By solving Equation 10, two values of $x$ are obtained:

$$
x=\frac{-d \pm \sqrt{d^{2}-4 c e}}{2 c}
$$

From the two $x$ values from Equation 11, two relative coordinates $y$ can be easily obtained from Equation 9. Thus, two candidate estimated locations are obtained. However, only one from the two estimation results is the right answer. If only two TDOA measurements are obtained, it is impossible to select which set of coordinates is the right answer without any restrictive conditions on the nonlinear equations as described in section 2.2. It can be proved in this way.

Assuming there is a source node located at $\left(x_{s 1}, y_{s 1}\right)$, and three reference nodes $\left(x_{0}, y_{0}\right)$, $\left(x_{1}, y_{1}\right)$, and $\left(x_{2}, y_{2}\right)$, two TDOA measurements can be obtained from the three reference nodes. After calculating the nonlinear equations, two sets of coordinates, assumed to be $\left(x_{s 1}, y_{s 1}\right)$ and $\left(x_{s 2}, y_{s 2}\right)$, can be gained. Also assume that another source node is located at the coordinates $\left(x_{s 2}, y_{s 2}\right)$, as Figure $3-1$ shows. If the source node $\left(x_{s 2}, y_{s 2}\right)$ triggers a location identification process, the exact same two sets of coordinates can be obtained, 
because the same two TDOA measurements as the source node $\left(x_{s l}, y_{s l}\right)$ should be obtained. That is to say, based on the two TDOA measurements, the ambiguity can never be solved by the TDOA method itself.
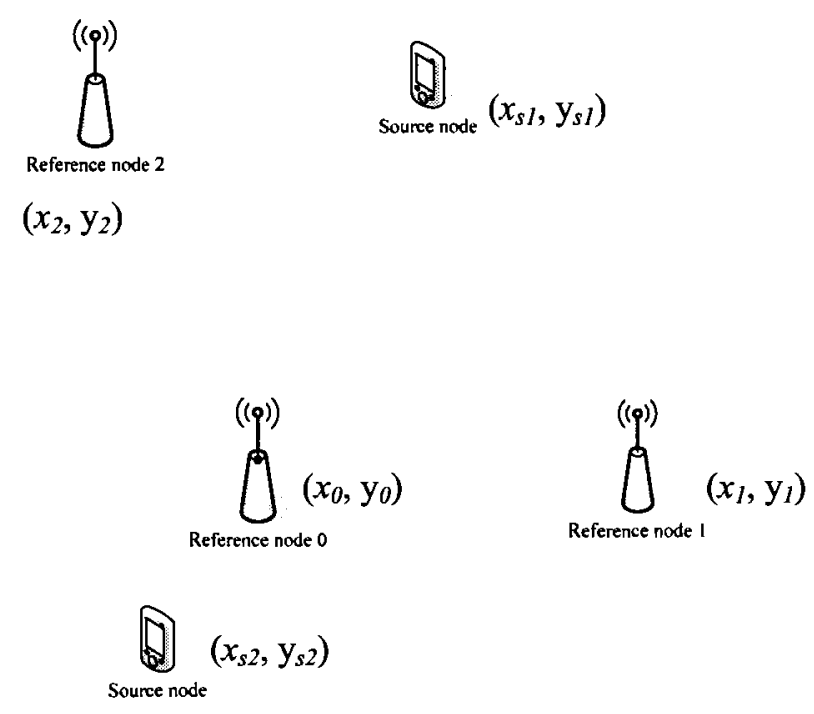

Figure 3-1 Ambiguity of the TDOA method

In wireless cellular systems, this ambiguity is not a problem, because one of the results will not belong to the cell the source node is in [46]. In fact, in wireless cellular systems, the cell that the source node belongs to is naturally restrict to the two results. The one that is inside the cell is the correct estimation. In Ad Hoc/Sensor networks, there are no such natural conditions to provide an exact restriction to the two results calculated from the nonlinear equations. Section 2.2 provides a survey of those works. All in all, those papers try to employ extra assumptions $[34,35]$ or assistant system $[36,37,38]$ to solve this ambiguity. An novel solution to this ambiguity is given in the hybrid TDOA method that does not need extra requirements on an Ad Hoc/Sensor network. By 
employing the Cell ID location estimation method in the Hybrid TDOA method, this problem is solved.

\subsection{Cell-based Location Identification System}

Cell-based location identification systems were introduced in Section 2.1.3. In wireless cellular systems, all reference nodes (called Base Stations in GSM and CDMA systems) are pre-organized, forming a hexagonal structure as shown in Figure 3-2.

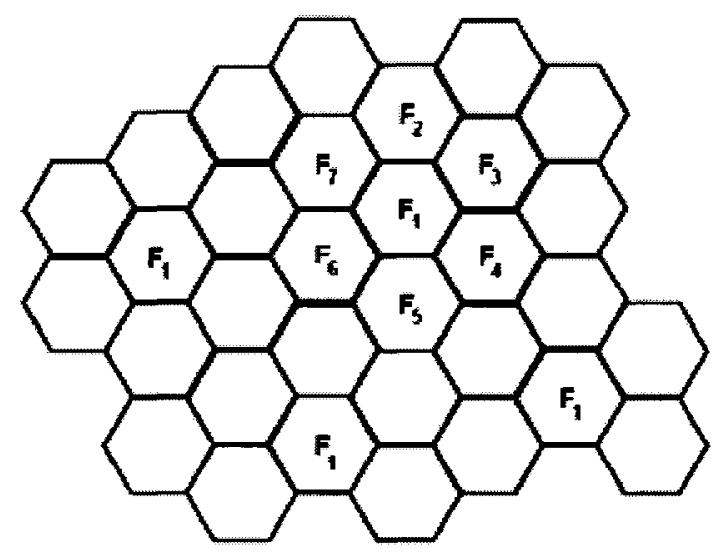

Figure 3-2 Cellular system

As described in Section 2.1.3, the cells in Ad Hoc/Sensor networks can be isolated into different types of areas, as shown in Figure 2-4 and Figure 3-3. 


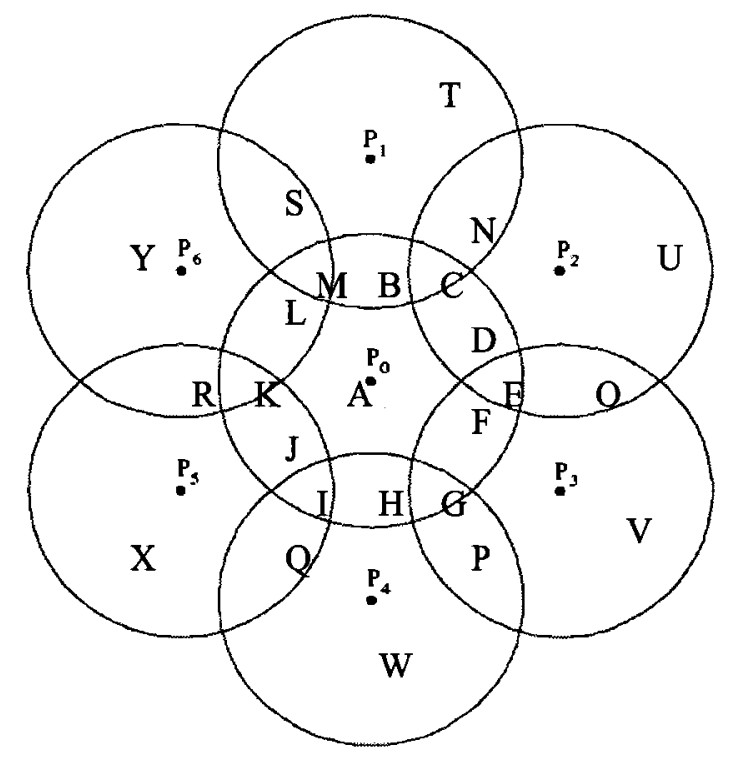

Figure 3-3 Types of areas in cells of Ad Hoc networks

There are 25 unique areas in Figure 3-3, A to Y. A mobile node can identify itself in an area by receiving the $R F$ signals of those reference nodes. Considering the hexagonal structure shown in Figure 3-3, those areas can be divided into three types, as follows:

- Type 1: An area covered by only one $R F$ signal of a reference node; i.e., area A, and areas $\mathrm{T}$ to $\mathrm{Y}$.

- $\quad$ Type 2: An area covered by two $R F$ signals from two reference nodes; i.e., areas B, D, F, H, J, and L.

- $\quad$ Type 3: An area covered by three $R F$ signals from three reference nodes; i.e., areas $\mathrm{C}, \mathrm{E}, \mathrm{G}, \mathrm{I}, \mathrm{K}$, and $\mathrm{M}$.

In fact, in Ad Hoc/Sensor networks, all nodes (source nodes and reference nodes) are arbitrarily distributed through the network area. Thus, there are more than three types of area. Some areas may be covered by more than three $R F$ signals from more than three reference nodes. Using simple geometry, the center point $\left(x_{c}, y_{c}\right)$ of those areas can be 
easily obtained. The following assumptions are held: The coordinates of reference point $P_{i}$ as $\left(x_{i}, y_{i}\right)$, every reference node has the same radio communication range, and $P_{i}$ is arbitrarily distributed in the two-dimensional space, thus, center point of an area covered by $N$ reference nodes from $i=1$ to $N$ can be expressed as follows:

$$
\left(x_{c}, y_{c}\right)=\left(\frac{x_{1}+x_{2} \cdots+x_{N}}{N}, \frac{y_{1}+y_{2} \cdots+y_{N}}{N}\right)
$$

If the assumption that each reference node has the same communication area is not held, assuming $P_{i}$ has the communication range as $R_{i}$, the following equation can be easily obtained:

$$
\left(x_{c}, y_{c}\right)=\left(\left(\frac{R_{1}}{\sum_{i=1}^{N} R_{i}} x_{1}+\frac{R_{2}}{\sum_{i=1}^{N} R_{i}} x_{2}+\cdots+\frac{R_{N}}{\sum_{i=1}^{N} R_{i}} x_{N}\right),\left(\frac{R_{1}}{\sum_{i=1}^{N} R_{i}} y_{1}+\frac{R_{2}}{\sum_{i=1}^{N} R_{i}} y_{2}+\cdots+\frac{R_{N}}{\sum_{i=1}^{N} R_{i}} y_{N}\right)\right)
$$

In Ad Hoc/Sensor networks, the types of cell are more complex than the analysis we give above. In hybrid TDOA method, we do not identify a source node into certain cells, Only the central point of the cell that the source node belongs to is identified, which can provide enough information to solve the ambiguity problem.

\subsection{The Influence of Geometric Dilution of Precision in Location Identification Systems}

The Geometric Dilution of Precision (GDOP) is a navigation or positioning system term used in geometric engineering to describe the geometric strength of the reference node configuration on the accuracy of the estimated location. 


\subsubsection{Basic Idea of Geometric Dilution of Precision}

This concept can be understood from the following figures. Errors in TDOA positioning systems are introduced by many issues [22, 23], like interference, quantization of time synchronization, etc. Disregarding the above issues, let us consider the influence of geometrics in a simple triangulation.

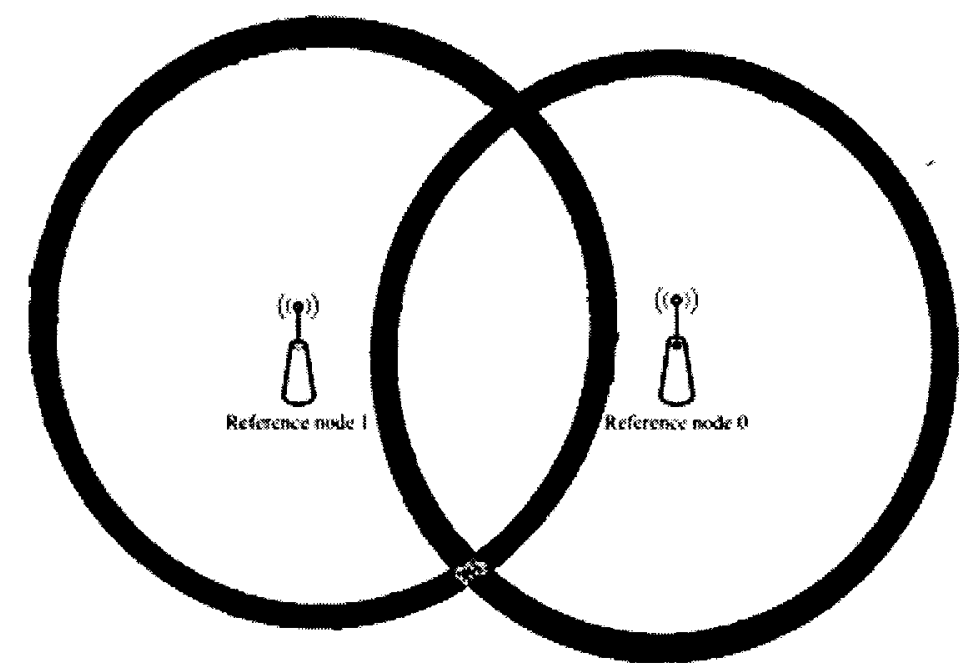

Figure 3-4 Error space in TDOA measurement

In Figure 3-4, the black area of the circles represents errors introduced by all other issues in triangulation, and the dotted-overlapped area is the probable area of the source node's estimated location. The following figure shows the change in the black-dotted area if the relative positions between the reference nodes and the source node change. 


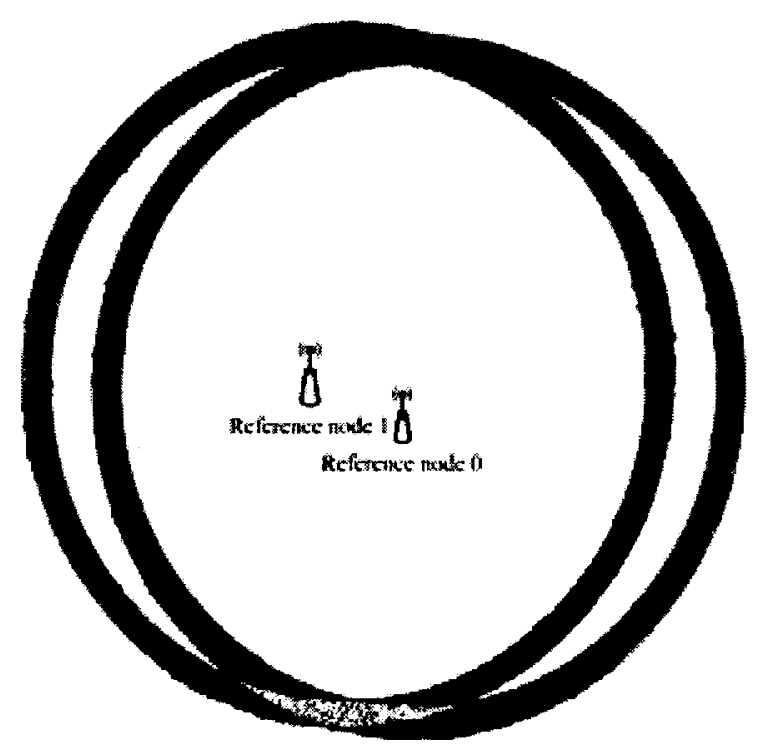

Figure 3-5 Error space in TDOA when relative positions have changed

In Figure 3-5, obviously, the black-dotted area is bigger than the black-dotted area in Figure 3-4. Thus, under the same error conditions for the other issues, the accuracy of the estimated location is decreased, because of the change of relative positions between the source node and the reference nodes.

In essence, the angle $(\theta)$, as the following figure shows, primarily influences the GDOP [48].

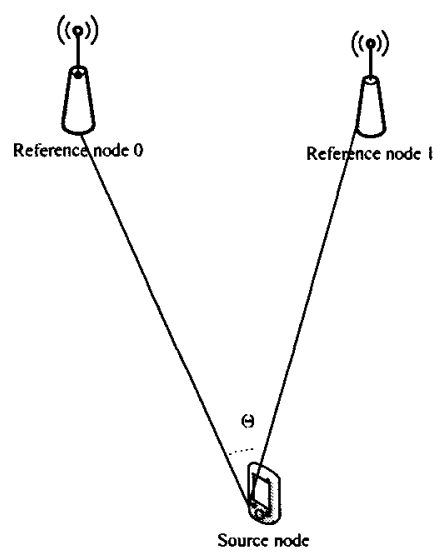

Figure 3-6 Key point of GDOP influence 


\subsubsection{Details of Geometric Dilution of Precision Influence}

In time-based wireless location systems (TOA, TDOA, etc.), the geometric formation of the locations of the reference nodes and the source node also affects the accuracy of mobile location estimation [49]. However, measuring GDOP is not a trivial task.

In order to describe the Hybrid TDOA method algorithm, the concept of refer-triangle is defined below. By connecting a set of three reference nodes, a triangle can be derived. In this thesis, such a triangle is called a refer-triangle, as shown in Figure 3-7.

This section illustrates other work in this area and shows that when the source node is outside a refer-triangle, the GDOP is higher than the GDOP when the source node is inside a refer-triangle.

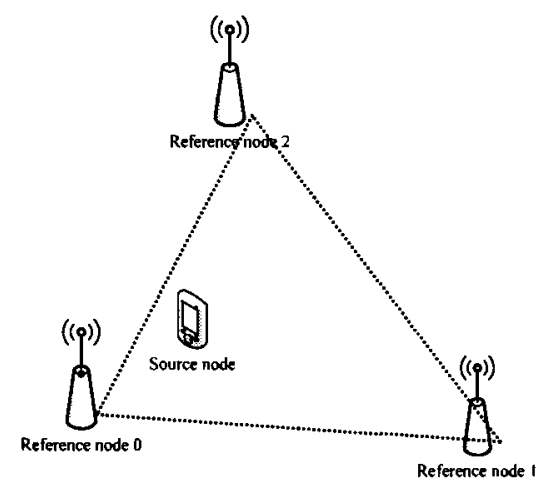

Figure 3-7 A typical refer-triangle

The GDOP provides a measure of the effect of geometric reference node formation on location estimation. For an unbiased estimator, the GDOP is defined as the ratio of the $r m s$ (root mean square) position error to the $r m s$ ranging error $[49,50,51]$. It can be calculated as follows [50,51]: 


$$
G D O P=\frac{\sqrt{E\left[\left(x_{s}-\mu\right)^{T}\left(x_{s}-\mu\right)\right]}}{\sigma_{\tau}}
$$

where $x_{s}$ is the estimated location, $\mu$ is the mean of the location estimations, and $\sigma_{\tau}$ is the $r m s$ ranging error for the TOA or TDOA measuring systems. In a TDOA system, a ranging error means an error introduced in the time of difference measurement. For a source node that is located at any position within the geometric reference formation, the values of GDOP vary accordingly. The ranging measurements suffer from thermal, instrument, and propagation errors, and so on. GDOP is very difficult to model. In [52], with the assumption that the ranging errors are random, independent, have zero mean, and have an identical rms value, the authors illustrated the GDOP in TDOA and TOA location identification systems in two-dimensional space, under different conditions, when $\sigma_{\tau}=1$. The following figures are from [52].
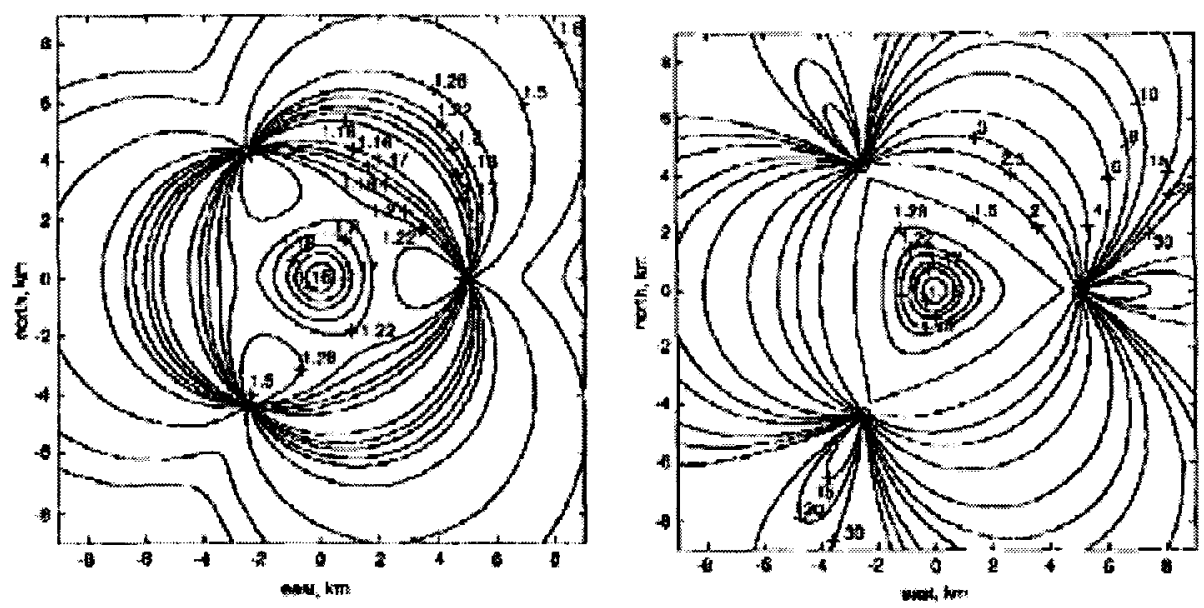

Figure 3-8 GDOP contours using three reference nodes 


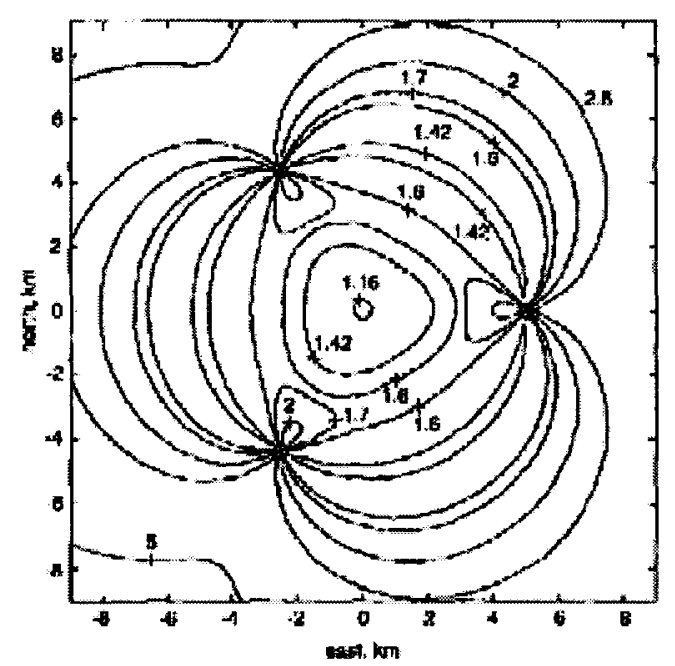

Figure 3-9 Normalized GDOP contours using three reference nodes

The above three GDOP contour maps show the two-dimensional GDOP of a 3-sided regular polygon. The values of those contours in above figures are calculated based on simulations in [52] and represent the contribution of GDOP to estimation errors. And the minimum value of GDOP is developed and proved by simulation in [52], which is 1.16 in a refer-triangle. Thus the final error of the estimation is the range error multiplies 1.16 , which means that 0.16 times the range error is the extra error introduced by GDOP exception range error. The above figures show that most of the contours of GDOP outside a refer-triangle are larger than 1.5 , which means GDOP will introduce extra $50 \%$ error from the range error.

From Figure 3-8 and Figure 3-9, it is clear that a source node outside the refer-triangle has a higher probability of obtaining a less accurate estimation than a source node inside the refer-triangle, because most of the GDOP values outside the refer-triangle are bigger than the GDOP values inside the refer-triangle. 
Thus, based on the previous research, a rough conclusion can be made that the accuracy of location estimation is lower if the source node is outside a refer-triangle than inside.

\subsection{Hybrid TDOA Location Identification Method}

This method combines the TDOA and Cell-based Location Identification methods, and employs GDOP theory, in which the accuracy of location identification is increased without increasing the cost of location identification service in an Ad Hoc/Sensor network and requiring additional conditions/assumptions.

\subsubsection{Hybrid TDOAAlgorithm}

In this section, the hybrid location identification algorithm is proposed. First, apart from the assumptions described in Section 3.1, some necessary assumptions are held for this algorithm. The rationality of those assumptions will be discussed later.

1. In an average spatial sense, all nodes are arbitrarily distributed following uniform distribution in a 2-D Euclidean plane.

2. Within a time duration that is short enough to allow a location identification process to be executed, all mobile nodes are fixed in certain positions, without moving. In other words, during a location identification process, the mobile nodes' speed and direction will not influence the location estimation accuracy.

The former two assumptions are reasonable in most common Ad Hoc/Sensor 
networks. Assumption 1 makes the hybrid TDOA method a two-dimensional location identification method. Of course, similar to the TDOA method itself, the hybrid TDOA method can be extended to 3-D space also. For assumption 2, considering that in this hybrid TDOA method, the $R F$ signal used to measure the time difference consists of the physical layer packets (in the simulation, IEEE802.11 series MAC protocol is employed), which means the process time of those packet is at the millisecond level, the speed of mobile nodes can be ignored in the Hybrid TDOA Algorithm.

This proposed Hybrid TDOA method provides a practical solution in Ad Hoc/Sensor networks with higher accuracy and those basic assumptions that are held in most kinds of Ad Hoc/Sensor networks, compared with the other solutions described in Section 2.2. The Hybrid TDOA method employs the Cell ID information to eliminate the ambiguity problem, moreover, by employing GDOP theory, the Hybrid TDOA solves the inconsistency problem in a new way, also provides higher accuracy in location estimation.

When a source node needs to locate itself, the source node will trigger a location identification process. The source node can figure out the TDOA measurements by the information of receiving time from reference nodes that received the source node's signal. After obtaining the TDOA measurements, the localization process can be done in the source node.

The hybrid TDOA method consists of the following main steps.

\section{Get cell information}


In this step, each mobile node first identifies itself belonging to certain cells, by obtaining a MAC packet from the one-hop neighbors' broadcast packet in Ad Hoc routing protocols. In those packets, a new section that records the reference node's position is necessary. In that way, a mobile node can identify the following facts:

a) How many reference nodes are among its one-hop neighbors;

b) What kind of cell it belongs to; and

c) The likely central point.

Moreover, the first step will only cost a new section to the MAC or the routing protocol packet that is regularly exchanged between one-hop neighbors.

\section{Refer-triangle selection for TDOA measurements}

When the source node needs to locate itself, the source node will trigger a location identification process by sending out a request packet. Those reference nodes that can receive the packet will send back the relative time from the time synchronizing system among reference nodes. After getting the information from the reference nodes, the source node will calculate the TDOA measurements and calculate the final estimated location. This step won't cost huge network resource, because only one packet sent out from the source node and each reference node will send back only one packet.

When there are more than three reference nodes received the signal from source node, source node will identify the relative positions between source node and each refer-triangle and delete the refer-triangles' candidate estimated results that have high probability to get low accurate estimated location. 
The relative position between the source node and refer-triangle can be briefly classified into three categories:

- The source node is outside the refer-triangle, as illustrated in Figure 3-10. We call it Category No. 1.

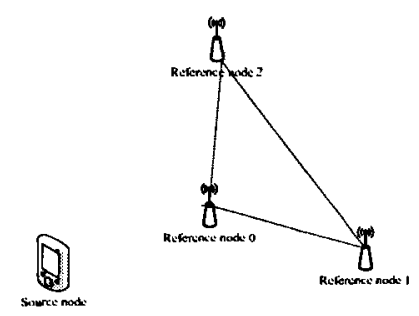

Figure 3-10 Category No. 1

- The source node is inside the obtuse refer-triangle, as illustrated in Figure 3-11. We call it Category No. 2.

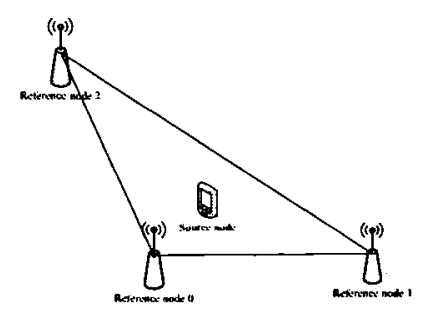

Figure 3-11 Category No. 2

- The source node is inside the acute refer-triangle, as illustrated in Figure 3-12. We call it Category No. 3.

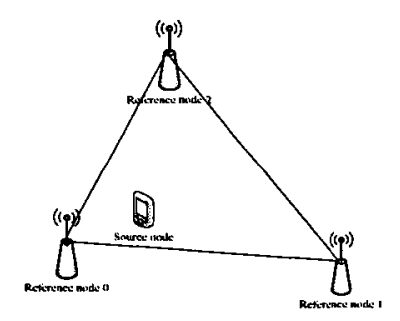

Figure 3-12 Category No. 3

Each of the former three categories can produce an estimation of the source node's 
location by calculating TDOA measurements. However, the accuracy of Category No. 1 will be far lower than Category No. 2 and Category No. 3. Hence, the estimation from Category No.1 will be discarded. A detailed comparison will be given in the Simulation chapter, in Section 4.3.1.

As described in Section 2.2.1, if there are $M(M>3)$ reference nodes that can receive the $R F$ signal from the source node, and in total, $C_{3}^{M}$ combinations of refer-triangle are derived. But different combination results in different level of accuracy in estimation. If those combinations that result in a less accurate estimation can be identified and deleted, the final estimation of the source node's location will obtain a more accurate estimation. The degree to which it can increase the accuracy is shown in the Simulation chapter.

If there are $M(M>3)$ reference nodes that can receive the $R F$ signal from the source node, in the hybrid TDOA method, the category No. 1 combinations will be disregarded in the total $C_{3}^{M}$ combinations. The final estimated location is the least square value of the selected combinations.

In cellular systems, because all the positions of the base stations (reference nodes) are preplanned, based on the cell information, a source node can easily identify the relative position between itself and base stations. But in Ad Hoc/Sensor networks, because all the reference nodes are distributed arbitrarily throughout the network, it is difficult to say whether the source node can identify the relative position based only on the cell information. If the source node cannot identify the relative position between itself and the refer-triangles based on cell information, after the nonlinear equations have been 
calculated, the estimated location of the source node is obtained based on each TDOA measurement from every refer-triangle. Based on the estimated location, the relative position between this refer-triangle and the source node is obtained with higher accuracy. If the relative position is in category No. 1 , this estimated result will be left out from the total combinations.

As described in Section 3.2.2, in order to reduce the complexity of those nonlinear equations, the coordinates of a certain refer-triangle will be replaced in the new coordinator system, in which one of the three reference nodes will be on the coordinates $(0,0)$ (the $0^{\text {th }}$ reference node), the second reference node will be on the right side of $X$ axis, and the last one will be placed in a position on quadrant I or quadrant II, based on the new coordinator system decided by the positions of first two reference nodes, as shown in the following figure (Figure 3-13):
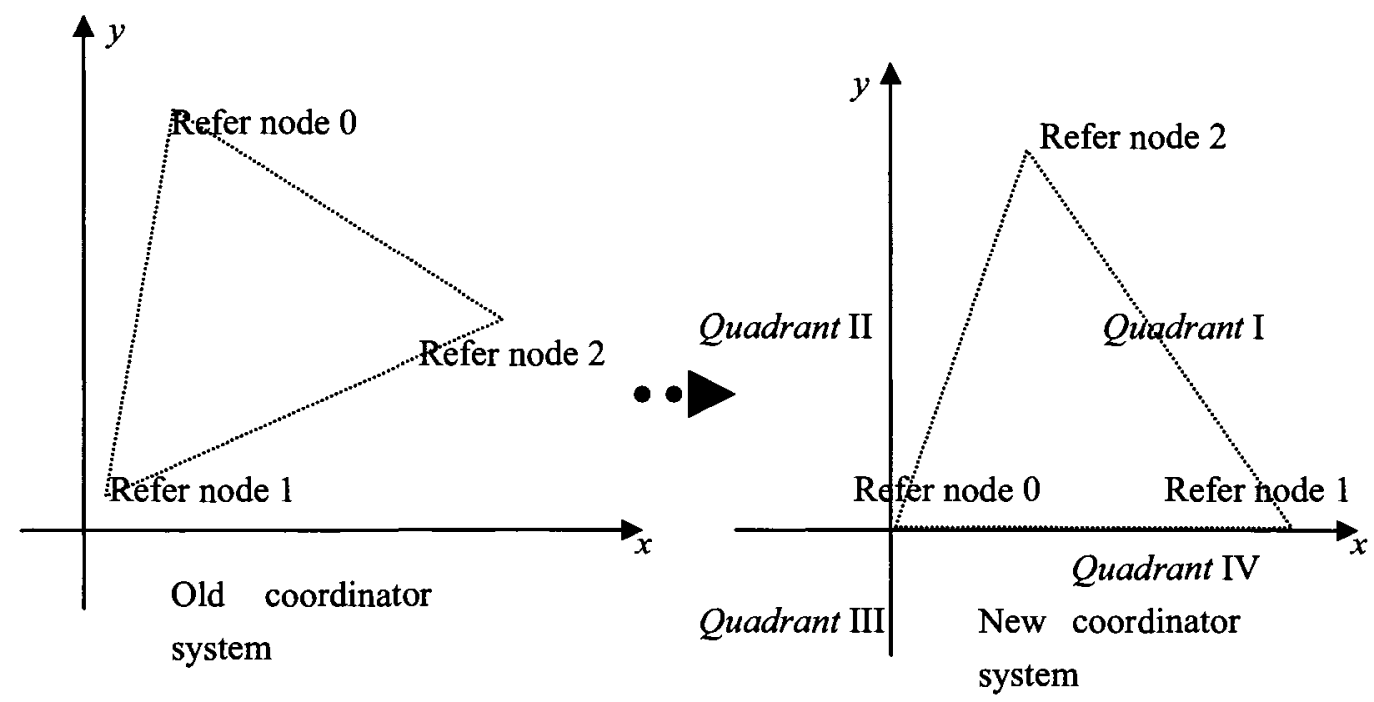

Figure 3-13 Coordinator systems exchange 
In the new coordinates system, the category of refer-triangles can be easily identified, after an estimation of the source node's position has been made based on this refer-triangle in the new coordinator system. The detailed algorithm for identifying the category of the refer-triangles is given in the pseudo code described in Appendix D. Moreover, two examples of pseudo code executing are presented in Appendix D also.

\section{Solution to the ambiguity problem}

As described in Sections 2.1.2 and 3.2.2, if there is only one refer-triangle, two sets of coordinates for the source node's location will be produced, after solving the nonlinear equations in Section 3.2.2. Furthermore, as described in Section 3.2.2, there is no possible way to pick a right answer from the two sets. In the hybrid TDOA algorithm, a solution that does not require extra assumptions or equipment is provided.

In the hybrid TDOA method, one set of the estimation results will be discarded by checking the location of the central point that is obtained in step 1 . The one closest to the central point will be selected.

If there is more than one refer-triangle (having more than three reference nodes receiving the source node's signal), each refer-triangle will produce two estimations. But only one set of estimations from the different refer-triangles will be close to each other. The other results will be distributed among the networks. An example can explain this clearly.

Assuming there are four reference nodes used in one location estimation process, as demonstrated in Figure 3-14. 


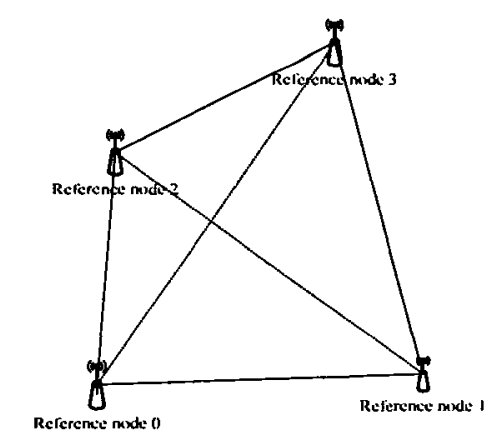

Figure 3-14 Four reference nodes

In this situation, there are four combinations of refer-triangles. Each of the four refer-triangle can provide two candidate location estimations as Figure 3-15 shown.
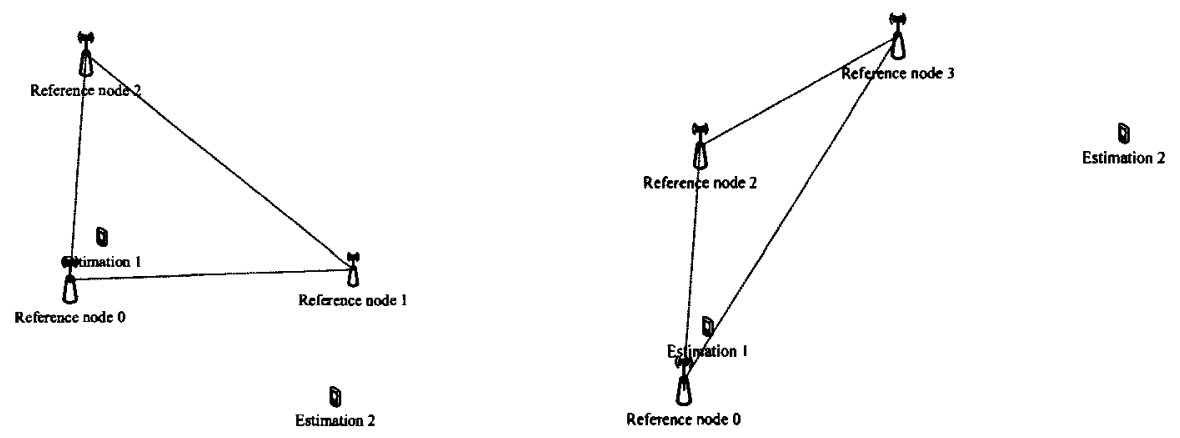

Figure 3-15 Candidate estimations in different refer-triangles

As the two charts in Figure 3-15 shows, the candidate estimation 1 deduced from refer-triangle (node 0 , node 1 and node 2 ) and the candidate estimation 1 obtained from refer-triangle (node 0 , node 2 and node 3 ) are near to each other, which means those two candidates are close to the real position of the source node. The other two candidates (estimation 2 from the two refer-triangles) are far from each other, which means they are far from the real location of the source node. Thus the estimation 1 from the two refer-triangles will be used to calculate the final estimation of the source node's position.

\section{Final location estimation}

If the source node needs to locate itself, it will trigger a location identification process 
by sending out a location identification $R F$ signal. Those reference nodes around the source node will then receive the signal and the TDOA measurement results can be obtained and sent back to the source node. Thus, the source node will make the calculation described in Section 3.2.2 for each selected combination of refer-triangle. After eliminating those results derived from the refer-triangles belonging to category No. 1, the final estimated coordinates of the source node's location is the least square value of those selected combinations' results. The following section proves that the least square value is exactly the average value of the selected combinations' results.

Assuming there are $N$ selected refer-triangles, there are $N$ possible locations of the source node. The least square method is used to find out the possible location $(P)$ that could minimize the following equation:

$$
\min \sum_{i=1}^{N}\left(P-P_{i}\right)^{2}
$$

where $P_{i}$ is the estimated location of refer-triangle $i$. Thus, this equation could be written in another way:

$$
\min \sum_{i=1}^{N}\left(\sqrt{\left(x-x_{i}\right)^{2}+\left(y-y_{i}\right)^{2}}\right)^{2}=\min \sum_{i=1}^{N}\left(\left(x-x_{i}\right)^{2}+\left(y-y_{i}\right)^{2}\right)
$$

In order to get the minimum value of the former equation, we set

$$
f(x, y)=\sum_{i=1}^{N}\left(\left(x-x_{i}\right)^{2}+\left(y-y_{i}\right)^{2}\right)
$$

The following steps are then taken: 


$$
\begin{aligned}
& \frac{\partial f(x, y)}{\partial x}=\frac{\partial \sum_{i=1}^{N}\left(\left(x-x_{i}\right)^{2}+\left(y-y_{i}\right)^{2}\right)}{\partial x}=2 \sum_{i=1}^{N}\left(x-x_{i}\right)=0 \\
& \Rightarrow \sum_{i=1}^{N} x=\sum_{i=1}^{N} x_{i} \Rightarrow x=\frac{\sum_{i=1}^{N} x_{i}}{N}
\end{aligned}
$$

Then, we also have the following:

$$
\begin{aligned}
& \frac{\partial f(x, y)}{\partial y}=\frac{\partial \sum_{i=1}^{N}\left(\left(x-x_{i}\right)^{2}+\left(y-y_{i}\right)^{2}\right)}{\partial y}=2 \sum_{i=1}^{N}\left(y-y_{i}\right)=0 \\
& \Rightarrow \sum_{i=1}^{N} y=\sum_{i=1}^{N} y_{i} \Rightarrow y=\frac{\sum_{i=1}^{N} y_{i}}{N}
\end{aligned}
$$

From the above deduction, it is clear that the average value of those selected refer-triangles' results is exactly the least square result of the estimated location of the source node. In the Hybrid TDOA algorithm, the average value of those selected refer-triangles' results will be used as the final location estimation. A computing performance comparison between Hybrid TDOA method and traditional LS TDOA method is given in Section 4.3.2. 


\section{Chapter 4 Simulation and Analysis}

This chapter describes the simulation, implementation in Network Simulator Version 2 (NS2) to validate the influence of GDOP over TDOA, simulation implementations for regular TDOA and the proposed Hybrid TDOA, and the simulation results analysis.

\subsection{Simulation Objectives}

The objectives in this simulation are as follows:

- Implement the regular least square TDOA method and the Hybrid TDOA method in a simulation environment

- Validate the effect of GDOP and the Hybrid TDOA method in simulation

- Analyze the performance of the Hybrid TDOA method in different scenarios in this simulation

- Compare the performance between the Hybrid TDOA method and the regular least square TDOA method in different scenarios in this simulation

\subsection{Simulation Implementation}

\subsubsection{Physical Model}

In [57], the authors surveyed many published network simulation results to point out 
the systemic flaws. Although not all simulation studies should use the most detailed radio model available, many defects are caused by a lack of details, especially in low physical-layer models. In this section, detailed information on the models at the physical layers deployed in this simulation is given, as well as the reasons why those models are used.

MAC Layer: In order to reflect the most common situations, in this simulation, the most popularly used MAC protocol in Ad Hoc/Sensor networks, IEEE $802.11 \mathrm{~b} / \mathrm{g}$, is deployed. Moreover, IEEE $802.1 \mathrm{lb} / \mathrm{g}$ is fully implemented in NS2 [56, 60]. Additionally, in [57], it has been verified in several cases that IEEE 802.11 can be correctly simulated in NS2.

Noisy Propagation Model: In NS2, three noisy propagation models have been implemented. They are the Free Space model, the Two-ray Ground Reflection model, and the Shadowing model [60]. These models are used to simulate the received signal power of each packet. A receiving threshold exists at the physical layer of each mobile node. When a packet is received, if its signal power is below the receiving threshold, it is marked as an error and dropped by the MAC layer. In this simulation, the Shadowing model is deployed, because, at present, this model is the most sophisticated and a more general and widely used propagation model $[58,60]$. A detailed introduction to these three propagation models is given in Appendix $\mathrm{C}$.

Energy Model: In order to guarantee the statistic of connectivity probability in the same environment, the constant energy model is deployed, in which each node has 
constant power throughout the simulation.

\subsubsection{Hybrid TDOA Method Implementation}

This section describes the implementation of the Hybrid TDOA algorithm. This algorithm is implemented in $\mathrm{C}++$. This algorithm gets the TDOA measurements from the NS2 simulator, then calculates the estimated location of the source node. Additionally, the $\mathrm{C}++$ code carries out some statistical tasks for analysis. The detailed implementation of the algorithm can be found in Appendix D.

The network environment is implemented in NS2. The detailed setting of the simulation environment can be found in the next section.

The process is illustrated in Figure 4-1.

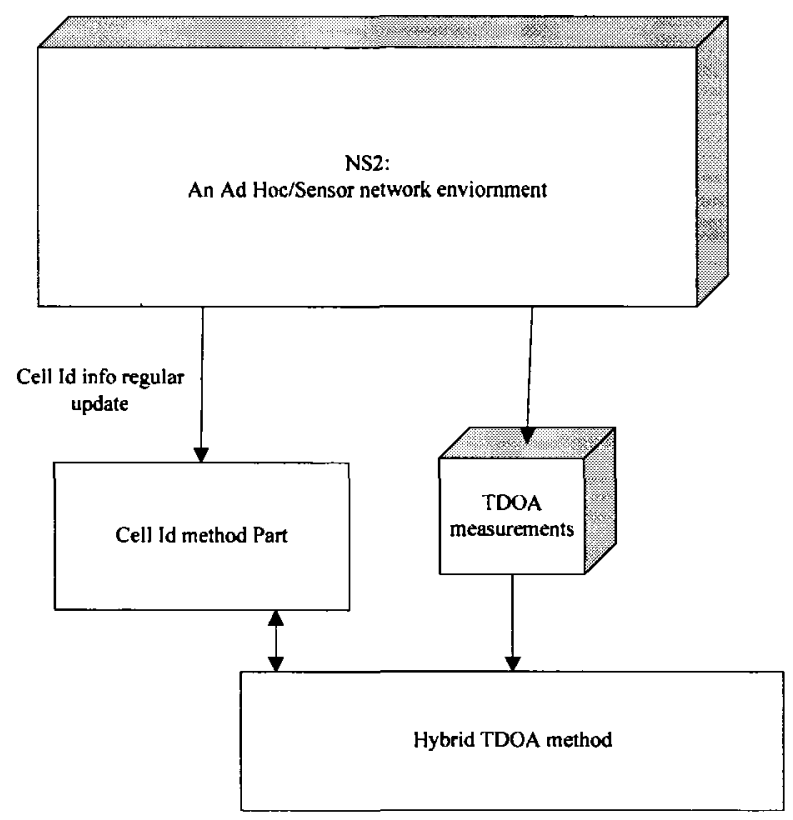

Figure 4-1 Hybrid TDOA method program models 


\subsubsection{Regular LS TDOA Method Implementation}

This section describes the implementation of the regular TDOA method in order to provide a comparison to our hybrid TDOA method. This algorithm is from [31, 33]. The basic LS (Least Square) TDOA is employed. This solution is also deduced from Equation 5 in Section 3.2.2.

Assuming $X_{i}=\left[x_{i}, y_{i}\right]^{T}$ denotes the $i^{\text {th }}$ reference node's coordinates $\left(x_{i}, y_{i}\right)$, and the source node's coordinates are $X_{s}=\left[x_{s}, y_{s}\right]^{T}$, then the distance from the source node to $i^{\text {th }}$ reference node $R_{i s}$ (Equation 3 in Section 3.2.3) can be denoted as $R_{i s}=\left\|X_{i}-X_{s}\right\|$. Thus, the distance between the $i^{\text {th }}$ reference node and the $0^{\text {th }}$ reference node (relocated in the $(0$, 0 ) in the new coordinates system, as described in Section 3.2.2) $R_{i 0}$ can be denoted as $R_{i 0}=\left\|X_{i}\right\|$. If $d_{i j}$ denotes the distance difference from reference $i$ and $j$, then the following equation can be obtained:

$$
d_{i j}=R_{i s}-R_{j s}=\left\|X_{i}-X_{s}\right\|-\left\|X_{j}-X_{s}\right\|
$$

If there are $M(M>3)$ reference nodes received the $R F$ signal of source node, $M-1 d_{j 0}$ can be determined and used in the following deduction.

From Equation 4 and Equation 16, the following equations can be derived directly.

$$
R_{i s}^{2}=\left(d_{i j}+R_{j s}\right)^{2}=d_{i j}^{2}+2 R_{j s} d_{i j}+R_{j s}^{2}
$$

And

$$
R_{i s}^{2}=\left\|X_{i}-X_{s}\right\|^{2}=R_{i o}^{2}-2 X_{i}^{T} X_{s}+R_{s o}^{2}
$$

Then, the following equation is obtained by subtracting Equation 18 from Equation 
17.

$$
\begin{aligned}
& 0=d_{i j}^{2}+2 R_{j s} d_{i j}+R_{j s}^{2}-R_{i o}^{2}+2 X_{i}^{T} X_{s}-R_{s o}^{2}=> \\
& 2 X_{i}^{T} X_{s}=R_{i o}^{2}-d_{i j}^{2}-2 R_{j s} d_{i j}+R_{s o}^{2}-R_{j s}^{2}
\end{aligned}
$$

If setting $i$ equals $j$, the $d_{j j}$ equals 0 . Then the following equation is obtained:

$$
2 X_{j}^{T} X_{s}=R_{j o}^{2}+R_{s o}^{2}-R_{j s}^{2}
$$

By subtracting Equation 20 from Equation 19, the following equation is obtained

$$
2\left(X_{i}-X_{j}\right)^{T} X_{s}=\left(R_{i 0}^{2}-R_{j 0}^{2}\right)-d_{i j}^{2}-2 R_{j s} d_{i j}
$$

Re-write the former equation into matrix form, as follows:

$$
S_{j} X_{s}=\mu_{j}-R_{j s} \rho_{j}
$$

in which

$$
\begin{aligned}
& S_{j}=\left[\begin{array}{ll}
\left(x_{1}-x_{j}\right) & \left(y_{1}-y_{j}\right) \\
\vdots & \vdots \\
\left(x_{j-1}-x_{j}\right) & \left(y_{j-1}-y_{j}\right) \\
\left(x_{j+1}-x_{j}\right) & \left(y_{j+1}-y_{j}\right) \\
\vdots & \vdots \\
\left(x_{M}-x_{j}\right) & \left(y_{M}-y_{j}\right)
\end{array}\right] \\
& (M-1) \times 2
\end{aligned}
$$

$\mu_{j}=\frac{1}{2} \bullet\left[\begin{array}{c}\left(R_{10}^{2}-R_{j 0}^{2}\right)-d_{1 j}^{2} \\ \vdots \\ \left(R_{j-1,0}^{2}-R_{j 0}^{2}\right)-d_{j-1, j}^{2} \\ \left(R_{j+1,0}^{2}-R_{j 0}^{2}\right)-d_{j-1, j}^{2} \\ \vdots \\ \left(R_{M 0}^{2}-R_{j 0}^{2}\right)-d_{M j}^{2}\end{array}\right]$

$(M-1) \times 1$ 


$$
\begin{gathered}
\rho_{j}=\left[\begin{array}{c}
d_{1 j} \\
\vdots \\
d_{j-1, j} \\
d_{j+1, j} \\
\vdots \\
d_{M, j}
\end{array}\right] \\
(M-1) \times 1
\end{gathered}
$$

where $M$ is the total reference nodes used in the TDOA measurement, thus total $M-1$ TDOA measurements can be reduced. Then $M-1$ rows are obtained in above matrixes.

From $M-1$ TDOA measurements, the vectors $\rho_{j}, \mu_{j}$ are known. Moreover, the matrix only depends on the reference nodes' locations that are known. The unknown variables in Equation 21 are the vectors $X_{s}$ and $R_{j s}$, the distance between the source node and the $j^{\text {th }}$ reference node, which is a function of $X_{s}$.

$[31,33]$ employ a matrix $U$ to eliminate the "nuisance parameter" $R_{j s .} U$ is a matrix that has $\rho_{j}$ in its null space. The matrix is as follows:

$$
U_{j}^{(k)}=\left(I-Z^{k}\right) D_{j}
$$

where

$$
D_{j}=\left[\operatorname{diag}\left\{\rho_{j}\right\}\right]^{-1}=\left[\begin{array}{cccccc}
d_{i j} & & & & & 0 \\
& : & & & 0 & \\
& & d_{j-1, j} & & & \\
& & d_{j+1, j} & & \\
& 0 & & & : & \\
0 & & & & d_{M, j}
\end{array}\right]^{-1}
$$

The circular shift matrix $Z$ is: 


$$
Z=\left[\begin{array}{cccc}
0 & 1 & . . & 0 \\
. . & . . & . . & . . \\
. . & 0 & . . & 1 \\
1 & . . & . . & 0
\end{array}\right]
$$

Thus, the following property for any value of $k$ can be obtained

$$
D_{j} \rho_{j}=[1,1, \ldots, 1]^{T}
$$

and

$$
\left(I-Z^{k}\right) 1=1-Z^{k} 1=1-1=0
$$

Assuming $k=1$ and suppressing the superscript $\operatorname{in} U_{j}^{(k)}$, from the property of $U$ described above, the following is easily obtained:

$$
U_{j} S_{j} X_{s}=U_{j} \mu_{j}
$$

Then, the vector $X_{s}$ of the location of the source node can be calculated by employing any good least square equation solver on the linear set of Equation 23, such as OriginLab and LinPack. The final result regarding the estimated location of the source node is then given as:

$$
X_{s}=\left(S_{j}^{T} U_{j}^{T} U_{j} S_{j}\right)^{-1} S_{j}^{T} U_{j}^{T} U_{j} \mu_{j}
$$

By using Equation 24, the final estimation can be calculated. Now, we can analyze the algorithm time complexity between the Hybrid TDOA method and traditional LS TDOA method, in order to give a basic idea on the computing efficiency between the two methods.

The $S_{j}$ matrix is a $n \times 2$ matrix and $U_{j}$ is a $n \times n$, where $n$ equals to $M-1$. The time complexity of any multiplication between such two matrices is $\mathrm{O}\left(n^{2}\right)$, because at least 
two loops in the program code can solve it. Thus, the time complexity of multiplication is $\mathrm{O}\left(n^{2}\right)$. Moreover to a matrix conversion, the time complexity is analyzed before, a basic introduction on it can be found in [62]. In [62], it shows that the time complexity of a matrix conversion operation in computing is at least $\mathrm{O}\left(n^{2.376}\right)$, by using the Coppersmith-Winograd algorithm. The time complexity of other algorithm on matrix conversion is between $\mathrm{O}\left(n^{2.376}\right)$ and $\mathrm{O}\left(n^{3}\right)$. In a nutshell, the time complexity of Equation 24 will be between $\mathrm{O}\left(n^{2.376}\right)$ and $\mathrm{O}\left(n^{3}\right)$, depending on what kinds of algorithm is used to calculate the matrix conversion. Therefore, the time complexity of traditional LS TDOA method is also between $\mathrm{O}\left(n^{2.376}\right)$ and $\mathrm{O}\left(n^{3}\right)$.

For the Hybrid TDOA method, the time complexity is $\mathrm{O}\left(n^{2}\right)$. The reason is as follows. Because there are $M-1$ TDOA measurements, $M-1$ nonlinear equations (Equation 6) will be produced as the following matrix shows.

$$
\begin{aligned}
& R_{i, 0}{ }^{2}+2 R_{i, 0} R_{0}=x_{i}{ }^{2}+y_{i}{ }^{2}-2 x_{i, 0} x-2 y_{i, 0} y-x_{0}{ }^{2}-y_{0}{ }^{2}
\end{aligned}
$$

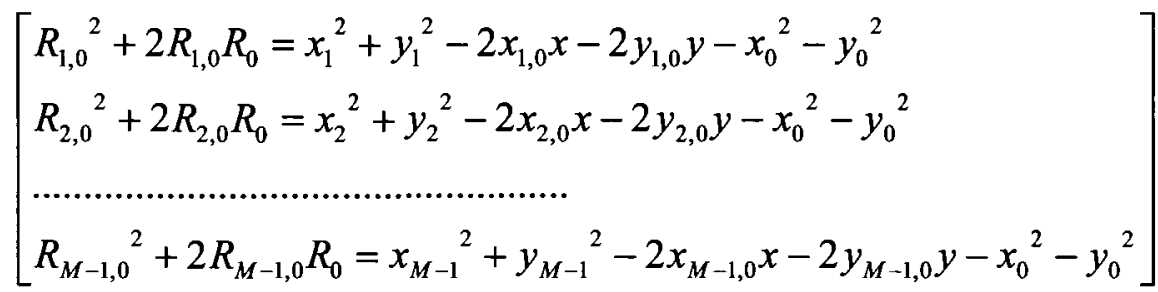

$$
\begin{aligned}
& (M-1) \times 1
\end{aligned}
$$

Each combination of two equations can deduce a candidate estimation, the final value will be the average value of all selected candidate estimations. To average out the computing operations, the time complexity is $\mathrm{O}(n)$, and two loops can reach all the combinations of the set of $M-1$ equations $\left(\mathrm{n}^{2} / 2\right.$ combinations). Thus the time complexity 
of Hybrid TDOA method is $T(f(n))=\frac{1}{2} n^{2}+n=O\left(n^{2}\right)$, where $f(n)$ is the matrix of equation 6 above. From the time complexity perspective, the Hybrid TDOA method is better than traditional LS TDOA method.

On the other hand, considering that different computational operations have different cost, for LS TDOA method, the main operation is the multiplication between a unit of matrix $S$ and $U$. For Hybrid TDOA method, on the other hand, the main operation is to solve a set of equations as follows.

$$
\left\{\begin{array}{l}
{R_{i, 0}}^{2}+2 R_{i, 0} R_{0}=x_{i}{ }^{2}+y_{i}{ }^{2}-2 x_{i, 0} x-2 y_{i, 0} y-x_{0}{ }^{2}-y_{0}{ }^{2} \\
R_{j, 0}{ }^{2}+2 R_{j, 0} R_{0}=x_{j}{ }^{2}+y_{j}{ }^{2}-2 x_{j, 0} x-2 y_{j, 0} y-x_{0}{ }^{2}-y_{0}{ }^{2}
\end{array}\right\}
$$

It is obvious, to a basic operation, the LS TDOA method will spend less time than the Hybrid TDOA method. Thus, we can see, when $n$ (equals to $M-1$ ) is very small, like $M-1=2$, the computing performance of the LS TDOA method is better than the Hybrid TDOA method. But with $n$ increasing, based on the different time complexities of the two methods, the Hybrid TDOA method will perform better than the LS TDOA method as $n$ increases.

To analyze the detailed computing performance in different situations is not a topic to the main concern of this thesis. The following sections will focus on the performance of accuracy of the two methods.

\subsubsection{Simulation Setting}

This section describes those detailed options in NS2 used to set the environment 
variables. The main options in NS2 used to set Ad Hoc network environment are as follows:

- opt(chan) defines the simulation nodes' channel type. It is set to wireless channel;

- opt(prop) defines the propagation model used in simulation. It is set to Shadowing Propagation Model;

- opt(netif) defines the node's network interface, in Ad Hoc network. It is set to physical wireless interface;

- $\quad \operatorname{opt}(\mathrm{mac})$ defines the MAC layer. Here it is IEEE802.11;

- opt(ant) defines the node's antenna type. It is set to Omni-Antenna;

- $\quad$ opt(ifq) defines the queue type deployed in this simulation. It is set to drop tail queue; and

- $\quad$ opt(li) defines the link layer type.

The above options are only those key variables used in Ad Hoc network simulation. Most other options used in the simulation are ignored in this section, but detailed information on those options can be found in Appendix C and also in [56]. Considering the NS2 simulates the whole network in real conditions, the queuing delay and MAC delay will also influence the accuracy. But the two kinds of delays are not analyzed in the chapter, because they have same influence to TDOA measurements that are used in both traditional LS TDOA method and Hybrid TDOA method, thus have no influence on the comparison between the two methods. 


\subsection{Performance of Regular TDOA and Hybrid TDOA in a Location Identification Process}

This section verifies the improvement in performance of the Hybrid TDOA method in a particular estimation process, which does not reflect the long-term average performance of the Hybrid TDOA method. In this section, several specific network topology scenarios are taken into account. In each estimation process, the accuracy of estimated result may vary at different levels. The standard deviation and mean of estimated results are used in this section to reflect performance.

\subsubsection{Influence of GDOP in TDOA}

Because the TDOA method is also based on triangulation, the relative position between the source node and the reference nodes (GDOP) will influence the location estimation accuracy [48 to 52]. This scenario is used to verify this result and to try to show that the refer-triangle belonging to category No. 2 and category No. 3 can derive a more accurate estimation than category No. 1 as described in section 3.4.2.

In this scenario, the number of reference nodes will be fixed at three; thus, only one refer-triangle exists. The relative position between source node and reference nodes is classified into three categories as described in section 3.5: the source node is outside the refer-triangle, the source node is inside the obtuse refer-triangle, and the source node is inside the acute refer-triangle. The three categories are shown as Figure 4-2: 


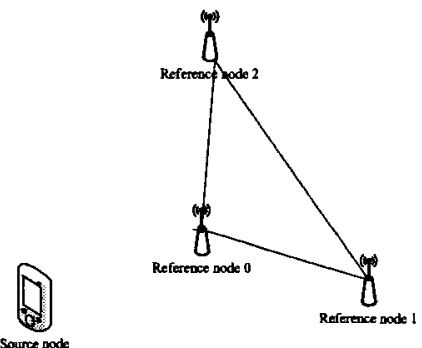

Category No. 1

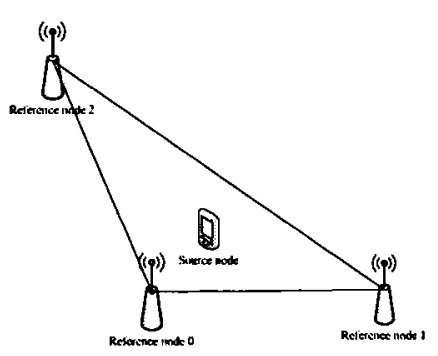

Category No. 2

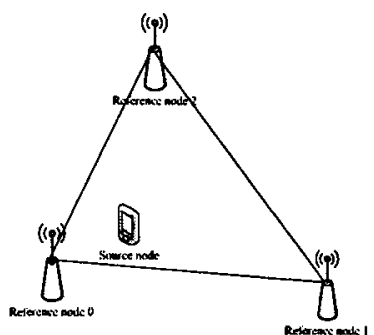

Category No. 3

Figure 4-2 Three categories of refer-triangle

There is a special case where all three reference nodes are on a line, which means the three reference nodes cannot derive a refer-triangle. Under this situation and if the source node is also on the segment of the line, as Figure 4-3 shows, this case is classified roughly in the second category. In the other situations, the source node can never be inside them, so this case is still classified in the first category.

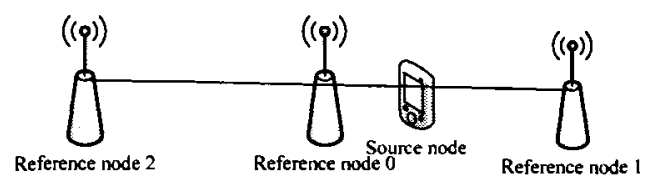

Figure 4-3 Category No. 2 special case

This simulation scenario is used to show the influence of GDOP on the accuracy of the estimated location. However, it only roughly shows that different categories of refer-triangle greatly influence the accuracy and identifies which kind of category can provide relatively better accuracy. It is impossible to simulate all possible positions to show all GDOP values. But this scenario shows clearly enough the strong influence of 
relative position and verifies which type of refer-triangle is best.

All three cases are executed in the same environment setting. In this simulation scenario, the location estimation accuracy is reflected by the Error Distance. The Error Distance is defined as the distance from the estimated location of the source node to the exact location of the source node. It can be obtained from the following expression, where DisError means the value of error distance, $\left(x_{E}, y_{E}\right)$ are the coordinates of estimated location of source node, and $(x, y)$ are the exact coordinates of the source node.

$$
D i s_{E r r o r}=\sqrt{\left(x_{E}-x\right)^{2}+\left(y_{E}-y\right)^{2}}
$$

The whole simulation chapter employs this concept in the same way as it is defined in this section, without special declaration. Moreover, throughout the whole chapter, the location identification process is triggered from the source node in regular time interval: 15 seconds.

The first run simulates the category No. 1 situation. Figure 4-4 reflects the DisError in 100 TDOA estimations following a time sequence, where three reference nodes are fixed in their location and the source node is moving randomly outside the refer-triangle. 


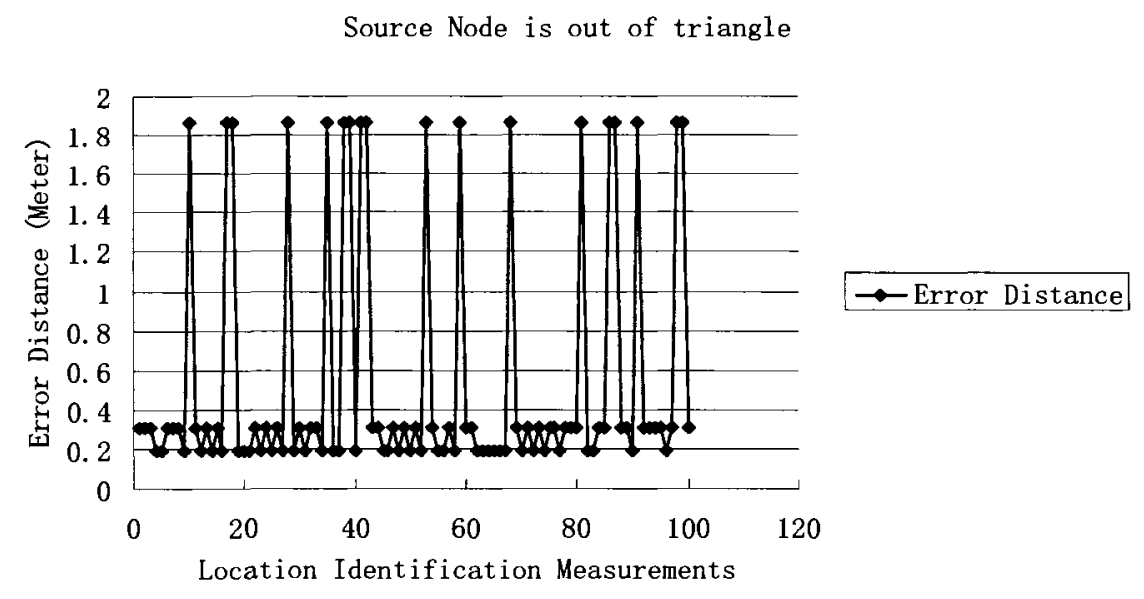

Figure 4-4 Source node is outside refer-triangle

It is clear, from Figure 4-4 that most TDOA estimations DisErrors range from 0.2 meters to 0.3 meters. However, 18 measurements derived larger errors (around 1.8 meters). The average DisError in this case is 0.542496 meters.

The second run reflects the category No. 2 situation. Figure 4-5 shows the DisError in 100 TDOA estimations following a time sequence, where three reference nodes are fixed in their location (an obtuse triangle) and the source node is moving randomly inside the refer-triangle.

Source Node is inside a obtuse triangle

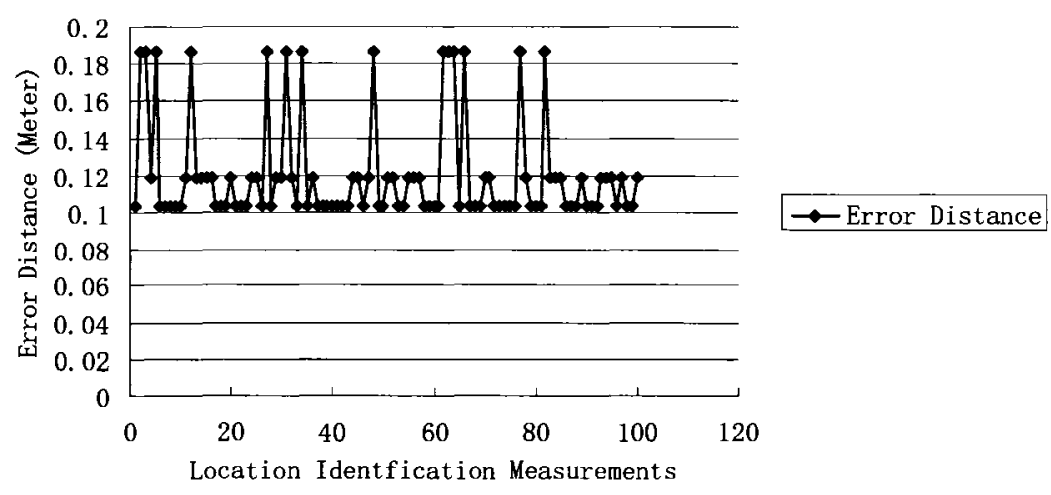

Figure 4-5 Source node inside a obtuse refer-triangle 
Figure 4-5 shows that most TDOA estimation distance errors range from 0.1 meters to 0.12 meters. However, 14 measurements derived larger errors, approximately 0.18 meters. The average DisError in this case is 0.119857 meters.

The third run simulates category No. 3. Figure 4-6 shows the error distance in 100 TDOA estimations following a time sequence, where three reference nodes are fixed in their location (an acute triangle) and the source node is moving randomly inside the refer-triangle.

Source Node is inside a acute triangle

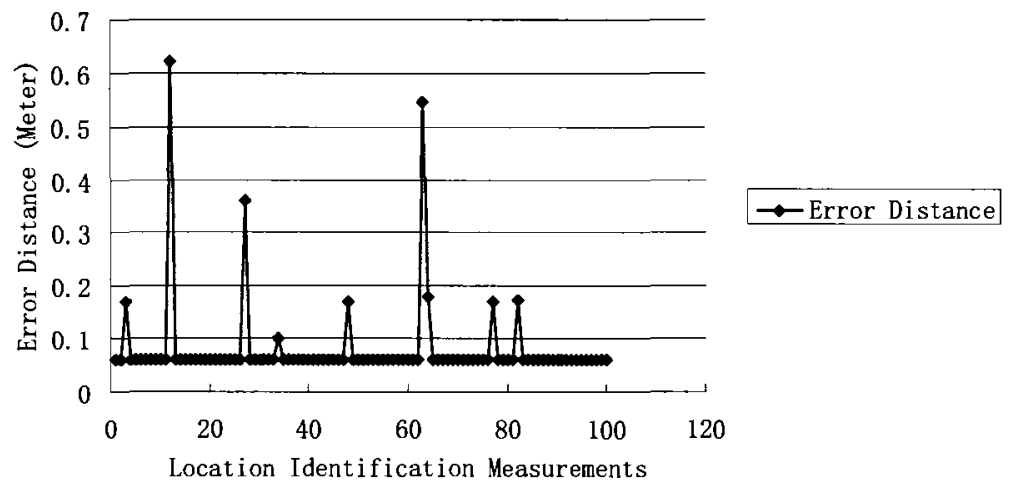

Figure 4-6 Source node inside an acute refer-triangle

This figure shows that most TDOA estimation distance errors are around 0.06 meters.

However, three measurements derived larger errors, from 0.4 meters to 0.6 meters. The average error distance in this case is 0.07982 meters.

Figure 4-7 compares the DisError among the three categories of relative position. 
Error Distance Comparison of three Categories

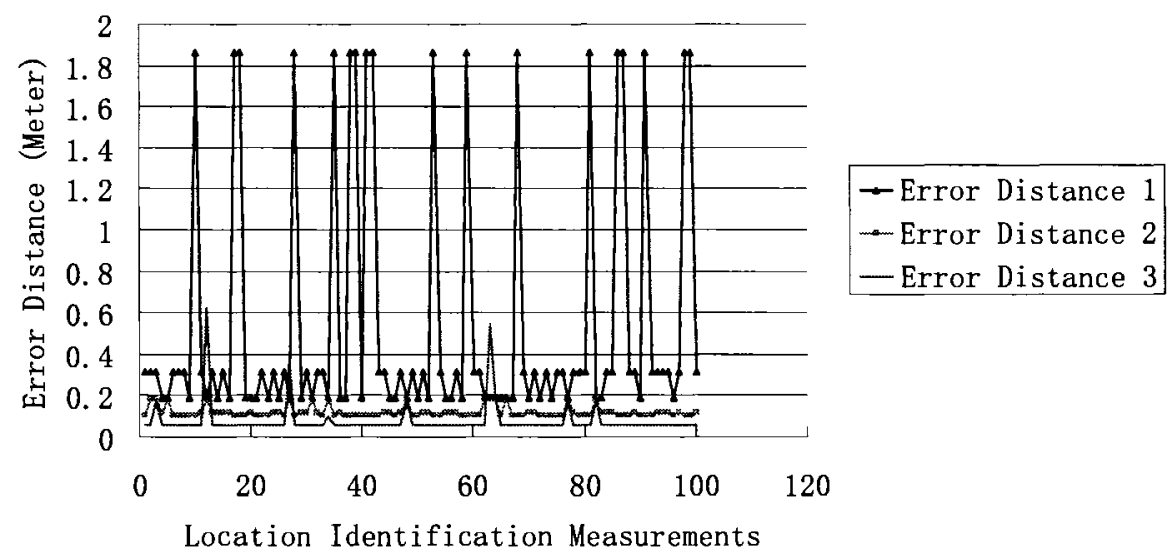

Figure 4-7 Error distance comparison of three categories

Figure 4-7 clearly proves that when the source node is inside an acute refer-triangle, the best estimation accuracy is derived, and when the source node is outside the refer-triangle, the worst estimated location is obtained. Moreover, considering those nodes (noted as exception points) that produce much larger error distances than the other points in all three cases, Figure 4-7 also shows that category No. 1 produced more exception points than the other two cases.

Table 4-1 and Table 4-2 show a detailed comparison of average DisErrors.

Table 4-1 Average error distances in three categories

\begin{tabular}{|l|c|c|c|}
\hline & $\begin{array}{l}\text { Average Error } \\
\text { Distance }\end{array}$ & $\begin{array}{l}\text { Exception point } \\
\text { percentage }\end{array}$ & $\begin{array}{l}\text { Average Error Distance of } \\
\text { exception points }\end{array}$ \\
\hline Category 1 & 0.542496 & $18 \%$ & 1.89473 \\
\hline Category 2 & 0.119857 & $14 \%$ & 0.185006 \\
\hline Category 3 & 0.07982 & $3 \%$ & 0.478201 \\
\hline
\end{tabular}

The standard deviation and mean DisError are derived from the table above: 
Table 4-2 Mean error distances and standard deviations in three categories

\begin{tabular}{|l|c|c|}
\hline & Mean Error Distance & Standard Deviation \\
\hline Category 1 & 0.542496 & 0.386 \\
\hline Category 2 & 0.119857 & 0.000762 \\
\hline Category 3 & 0.07982 & 0.006684 \\
\hline
\end{tabular}

Table 4-1 shows that the average error distance in category No. 1 is about five times larger than in category No. 2 and about seven times larger than in category No. 3 . The exception point in category No. 1 is about 1.5 times larger than in category No. 2 and six times larger than in category No. 3. For the average error distance of exception points, category No. 1 is 10 times larger than in category No. 2 and four times larger than in category No. 3. Although the average error distance of exception points of category No. 3 is twice the size of category No. 2 , the exception point percentage is four times smaller than category No. 2, and the average error distance of category No. 3 is 1.5 times smaller than category No. 1. From Table 4-2, the standard deviation of category No. 1 is much bigger than the other two categories, which means that, in a particular estimation process, category No. 1 has a much greater chance of obtaining an exception point that result in the DisError being much bigger than its average value. Table 4-1 and Table 4-2 show clearly that the best location estimation accuracy is obtained when the source node is inside a refer-triangle. Thus, the conclusion is that the estimation accuracy in the case where the source node is inside the refer-triangle is much better than the case where the source node is outside the refer-triangle. 


\subsubsection{More than Three Reference Nodes in a Estimation Process}

This scenario is designed to compare the influence of the number of reference nodes on the accuracy in an estimation process. It is common sense that more reference nodes would increase the estimation accuracy. However, from the simulation scenario in the previous section, it is known that the relative positions will greatly influence the location estimation. Considering the GDOP influence, things become more complex, and in certain source node and reference nodes topologies, the judgment that more reference nodes can derive a higher accuracy may not be always correct. The following simulation shows that a greater number of reference nodes does not always result in more accurate estimations.

\subsubsection{Analysis of Relative Position and Number of Reference Nodes in TDOA Method}

This first run employs three reference nodes in an area of $300 \mathrm{~m}^{2}$. The reference nodes are fixed in their locations, as Figure 4-8 shows, in which the source node is moving randomly inside the refer-triangle. In this scenario, the distance between each reference nodes is from $100 \mathrm{~m}$ to $220 \mathrm{~m}$ to ensure that the $R F$ signal from the source node can reach the three reference nodes. 


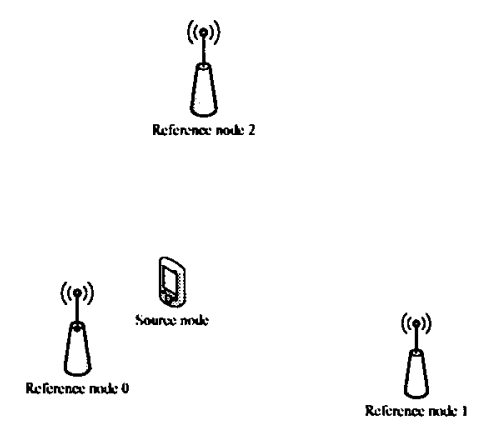

Figure 4-8 Three reference node situation

Figure 4-9 shows the simulation results.

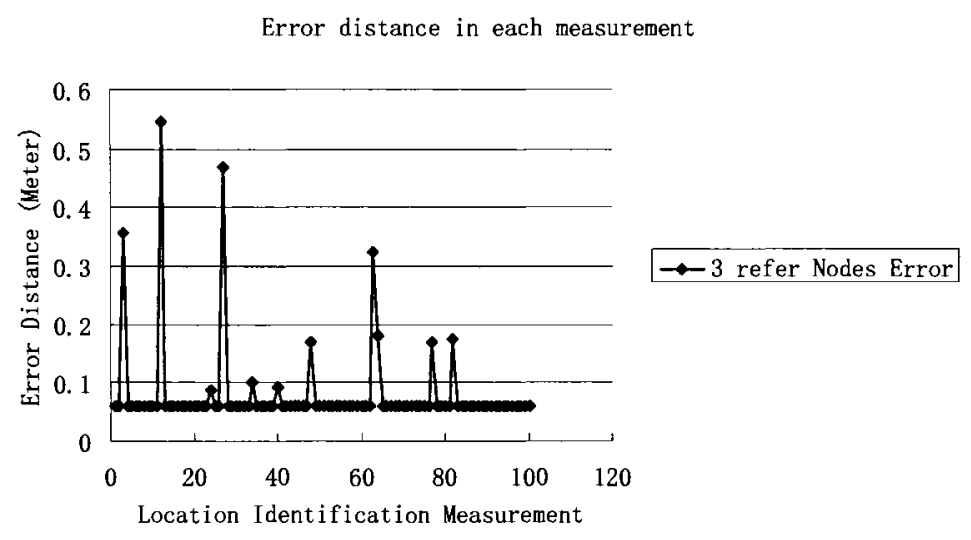

Figure 4-9 Error distance of three-reference-node situation

The second run employs four reference nodes $(M=4)$ in an area of $300 \mathrm{~m}^{2}$. The final estimated position will be calculated by the least square TDOA method, described in Section 4.2.3. The positions of the four reference nodes are fixed as shown in Figure 4-10. The source node moves randomly inside the rectangle. In this scenario, the distance between each pair of reference nodes is from $100 \mathrm{~m}$ to $220 \mathrm{~m}$ in order to make the $R F$ signal of source node can reach all reference nodes. 

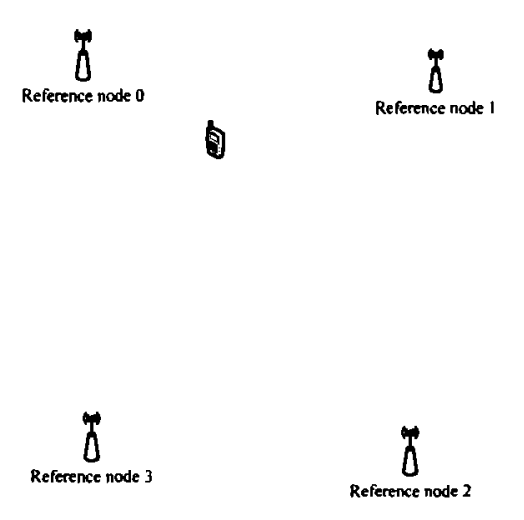

Figure 4-10 Four-reference-node situation

Figure 4-11 reflects the error distance in 100 TDOA measurements with four reference nodes.

Error distance in each measurement

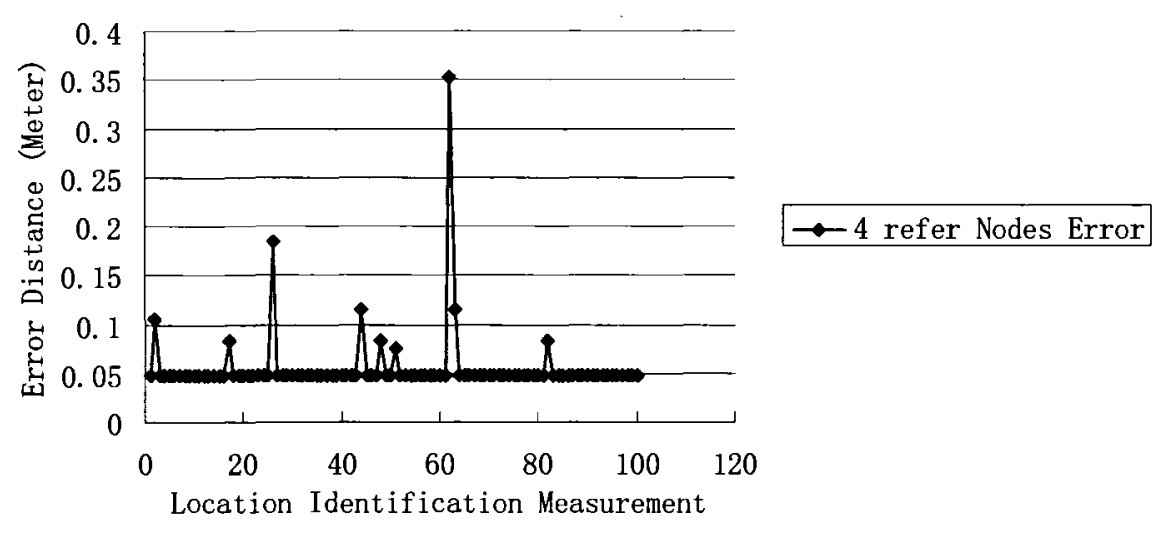

Figure 4-11 Error distance of four-reference-node situation

The third run employs five reference nodes in an area of $300 \mathrm{~m}^{2}$. The topology is described as shown in Figure 4-12. Considering the complexity of five reference nodes, the location of the source node is also fixed in its location, as Figure 4-12 shows. In this scenario, the distance between all pairs of reference nodes is from $100 \mathrm{~m}$ to $220 \mathrm{~m}$ to make ensure that the $R F$ signal of source node can reach all reference nodes. The more than five reference nodes situation will be reached in section 4.4 . 


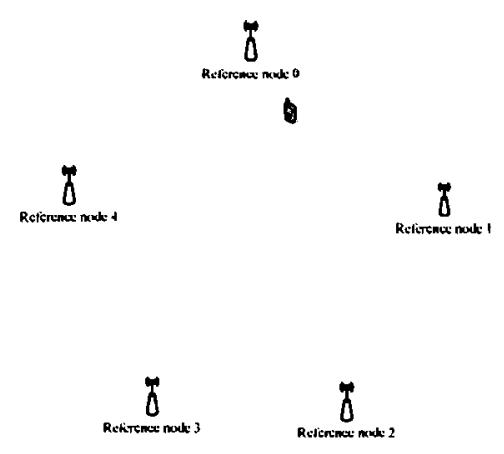

Figure 4-12 Five reference node situation

Figure 4-13 reflects the error distance in 100 TDOA measurements with five reference nodes.

Error distance in each measurement

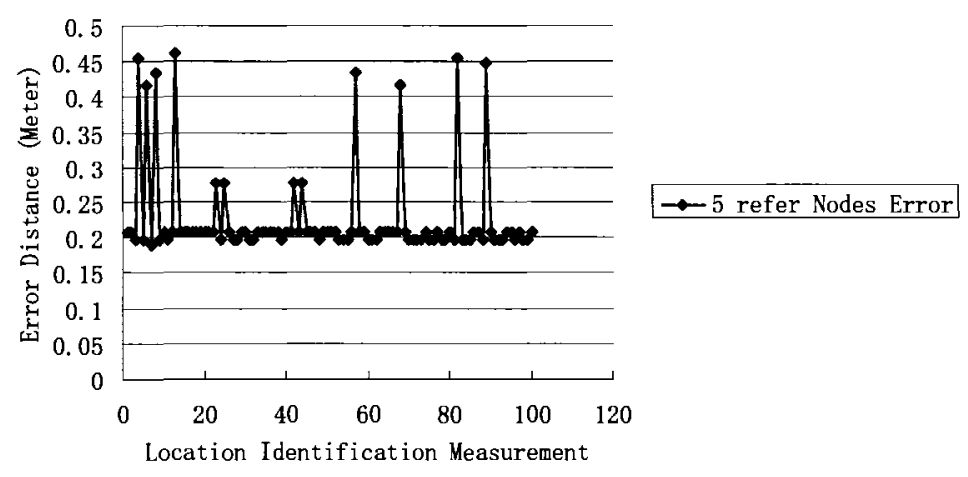

Figure 4-13 Error distance of five-reference-node situation

A comparison of the previous runs is shown in Figure 4-14.

Error distance in each measurement

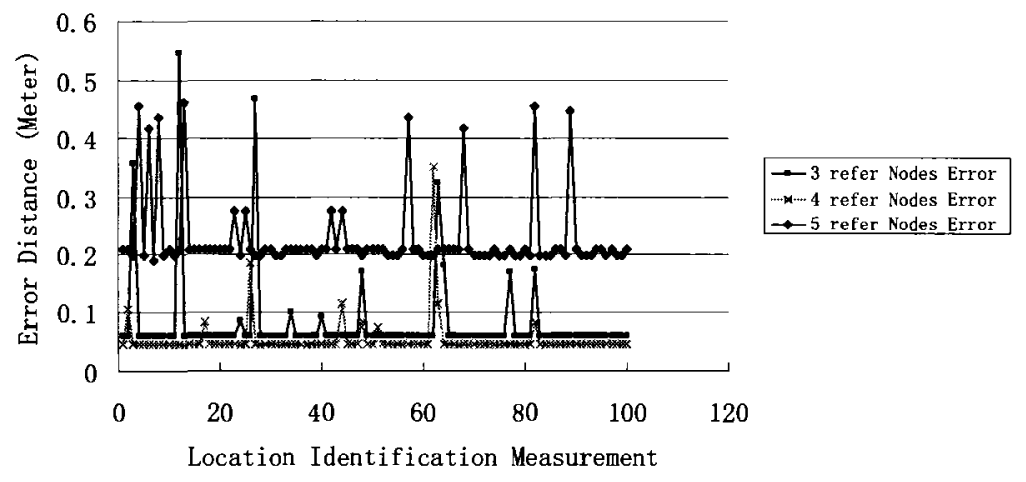

Figure 4-14 Error distance comparison of different number of reference nodes 
This clearly shows that the four reference node situation performs better than the three reference node situation, but the question is why the five reference node situation performs even worse than the three reference node situation.

Analyzing how that happened will be useful. For the five reference node situation, assuming a special case in which the five reference nodes produce a pentagon, as shown in Figure 4-15, and the source node is exactly in the center of the pentagon, there would be 10 combinations of a set of refer-triangles $\left(c_{3}^{5}\right)$, and five refer-triangles (dotted line connected triangles in those charts) belonging to category No. 1 in the previous section and five refer-triangles (solid line connected triangles in those charts) belonging to the other categories.
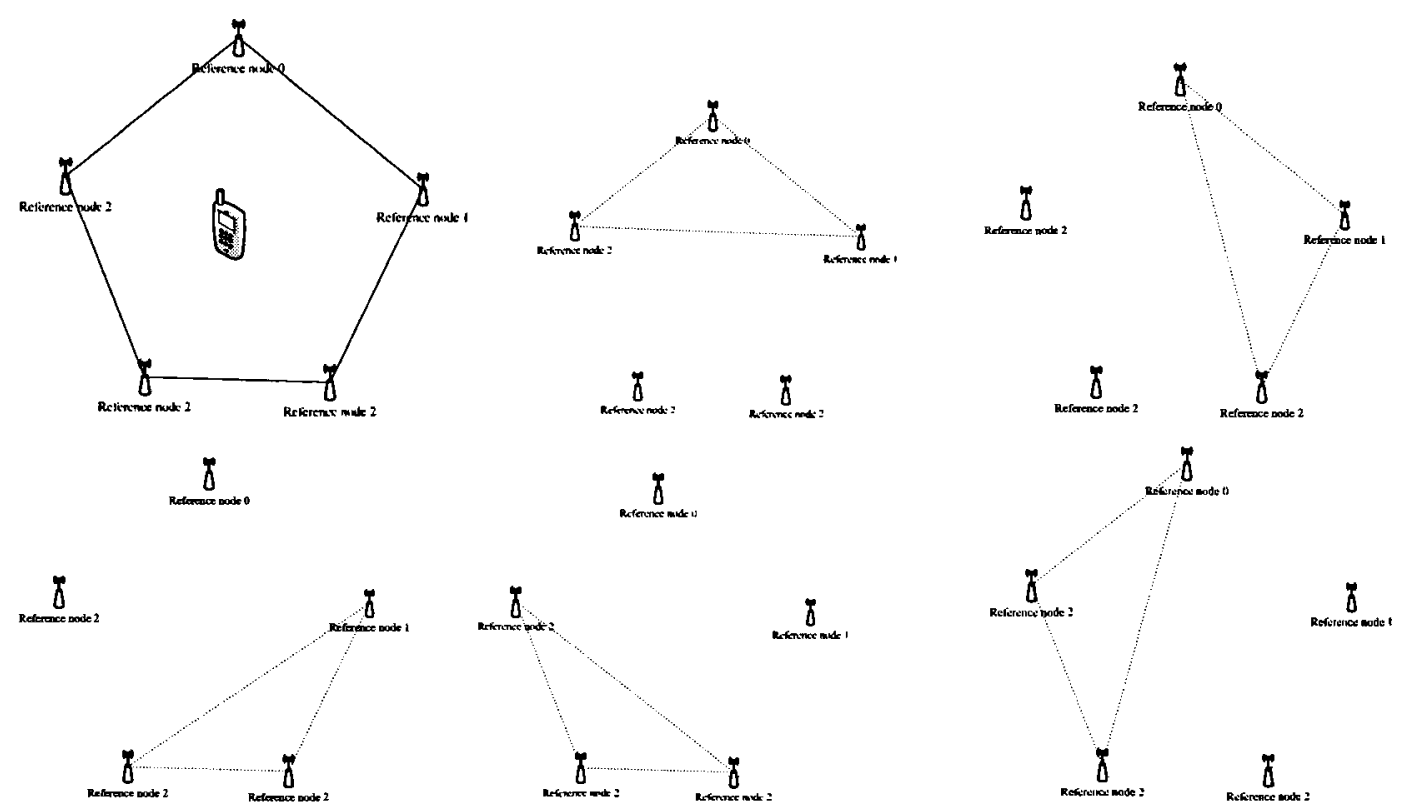

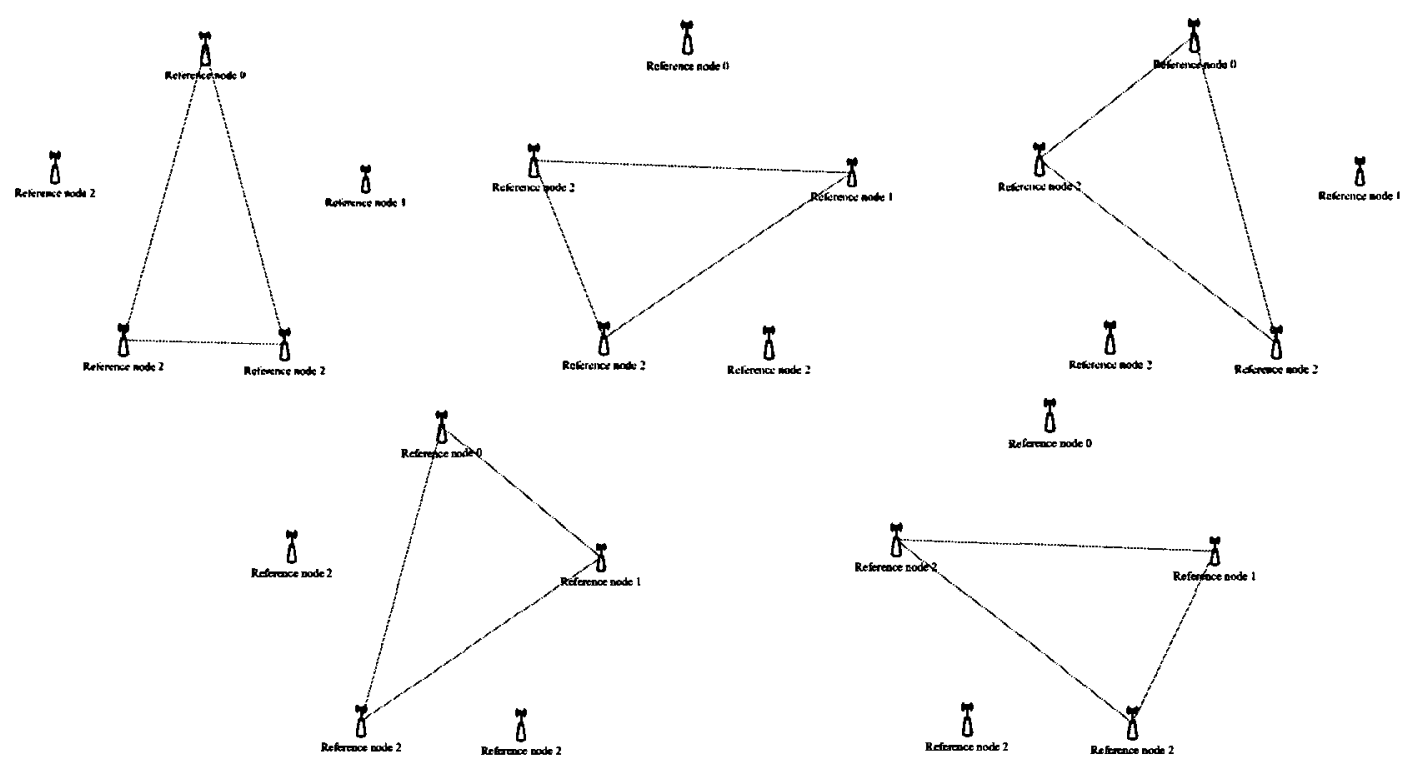

Figure 4-15 Different refer-triangles in five-reference-node situation

For the three-reference-node situation, there is only one refer-triangle. For the four-reference-node situation, there are $c_{3}^{4}=4$ refer-triangles. We focus on the complex situations of five reference nodes. Late on, an analysis on four-reference-node situation is also given. Based on the refer-triangles, the pentagon can be divided into 11 zones, as shown in Figure 4-16.

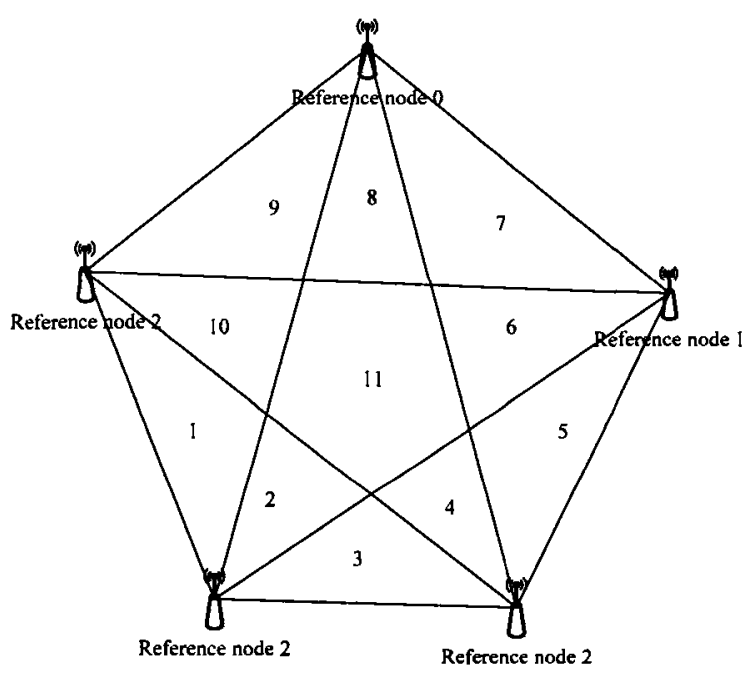

Figure 4-16 Refer-triangle classification in a five reference node situation 
At the beginning, the source node is assumed to be at the center of the pentagon; in other words, zone 11. Also the source node could be in zones 1 to 10 , or even outside the pentagon. If the source node is inside a zone, such as $1,3,5,7$, or 9 , only three refer-triangles belong to category No. 3, but all other eight refer-triangles belong to category No. 1 . If the source node is inside a zone such as $2,4,6,8$, or 10 , only two refer-triangles belong to category No. 3, but all other eight refer-triangles belong to category No. 1 .

Returning to the four reference node situation, assuming the four nodes can produce a quadrangle, as shown in Figure 4-17, there are two possible situations: if the source node is outside the quadrangle, a less accurate location estimation is obtained; if the source node is in one of the four zones $(1,2,3,4)$, two category No. 1 refer-triangles and two category No. 3 refer-triangles are obtained.

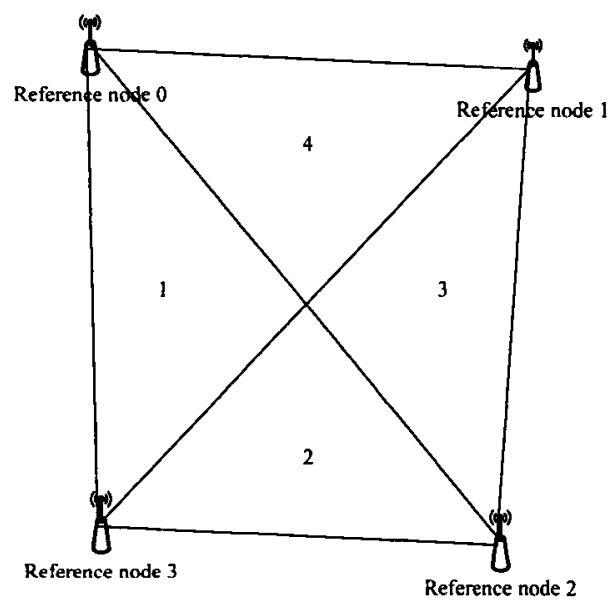

Figure 4-17 Refer-triangle classification in a four reference node situation

From the above analysis, it is clear that the estimation has a greater probability of obtaining more category No.1 refer-triangles in some situations, in some situations where 
more reference nodes can receive the source node's signal. That is why the five reference node situation performs worse than the three and four reference node situations.

\subsubsection{Comparison between LS TDOA and Hybrid TDOA in a Specific Case}

In the TDOA method, all TDOA measurements are employed. Because of the influence of GDOP, the estimation may result in an even worse location estimation than in the situations with less reference nodes. In our research, the hybrid TDOA method can avoid those refer-triangles belonging to category No. 1. If the estimated location belongs to category No. 1 , the estimated result of this refer-triangle will be ignored, and only those refer-triangles belonging to category No. 2 and category No. 3 will be counted. The following runs show a comparison between the regular LS TDOA method and the hybrid TDOA method in a specific scenario.

This simulation is based on three five-reference-node situations as Figure 4-16 shows (a pentagon):

- Situation 1: source node is in zone 11 (noted as Situation 1)

- Situation 2: source node is inside one of zones $1,3,5,7$ or 9 (noted as Situation 2)

- Situation 3: source node is inside a zone of zones $2,4,6,8$ or 10 (noted as Situation 3)

For the situation where the source node is outside the pentagon, there is a very low 
chance it will happen, because based on the assumption of this thesis that mobile nodes are distributed uniformly throughout the area, and every node used in this research has an omni-antenna, typically, a source node will find a series of reference nodes around itself. Only those nodes that are along the border of the area may encounter such a situation. Meanwhile, if the source node is outside the pentagon, the accuracy will not be good.

Figure 4-18, Figure 4-19 and Figure 4-20 show the DisError comparisons in the above three situations.

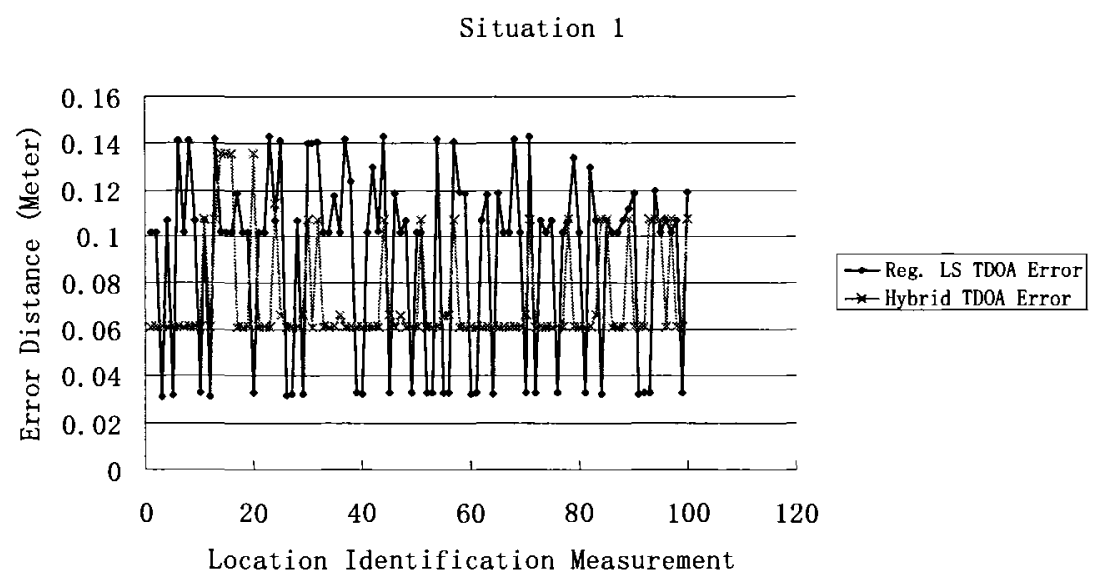

Figure 4-18 Error distance comparison for situation 1

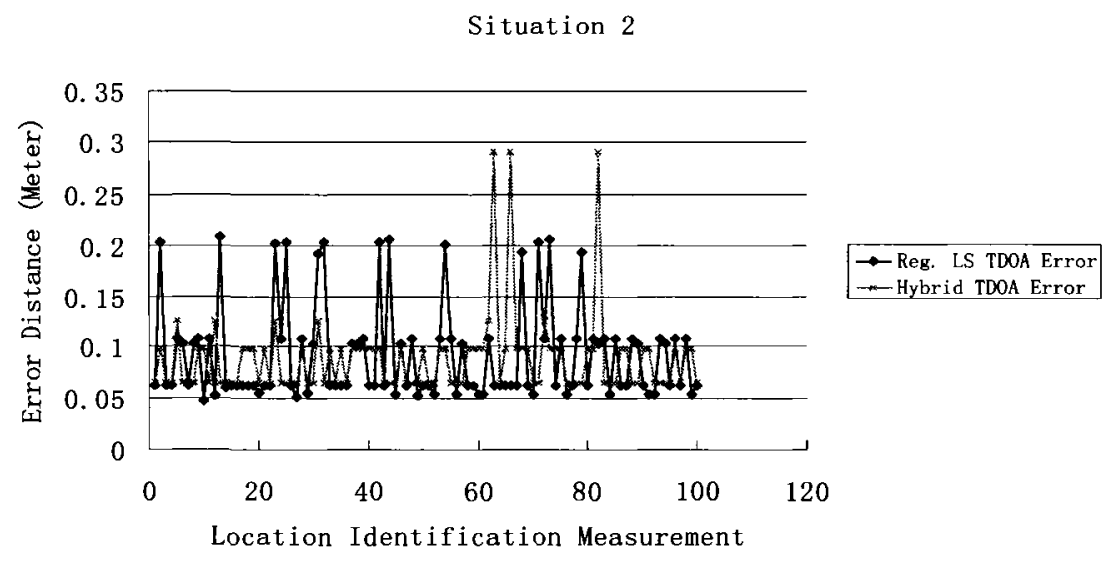

Figure 4-19 Error distance comparison for situation 2 
Situation 3

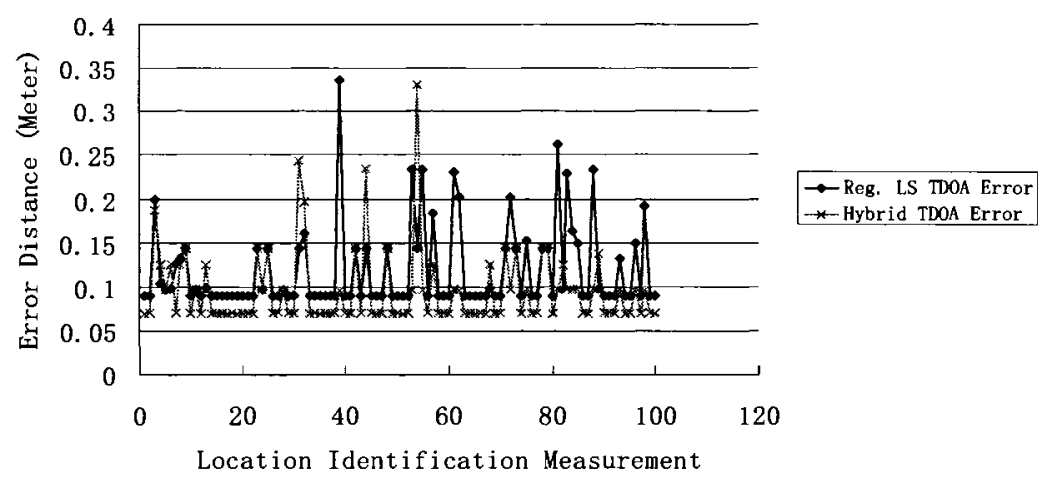

Figure 4-20 Error distance comparison for situation 3

From the above figures, the following table is obtained:

Table 4-3 Average error distances in different situations

\begin{tabular}{|l|c|c|c|l|}
\hline & $\begin{array}{l}\text { Ave. Error Distance } \\
\text { of Reg. LS TDOA }\end{array}$ & $\begin{array}{l}\text { Number of } \\
\text { category No. 1 } \\
\text { refer-triangles }\end{array}$ & $\begin{array}{l}\text { Ave. Error Distance of } \\
\text { Hybrid TDOA }\end{array}$ & $\begin{array}{l}\text { Number of } \\
\text { category No. 2 } \\
\text { and category No. } \\
\text { 3refer-triangle }\end{array}$ \\
\hline Situation 1 & 0.091573 & 5 & 0.072825 & 5 \\
\hline Situation 2 & 0.091953 & 7 & 0.088705 & 3 \\
\hline Situation 3 & 0.118182 & 8 & 0.094905 & 2 \\
\hline
\end{tabular}

The average DisError and standard deviations of the three situations are derived from

Table 4-3, as shown in Figure 4-21 and Figure 4-22.

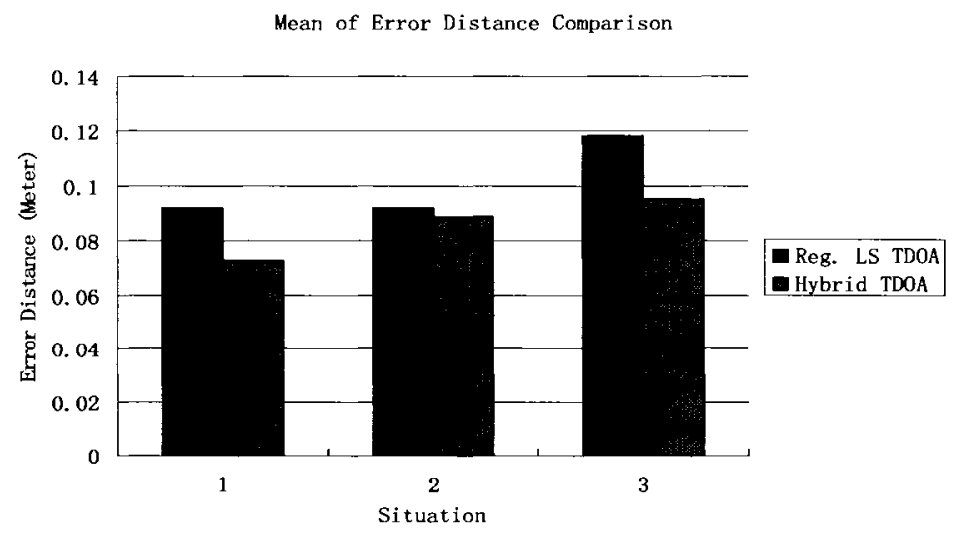

Figure 4-21 Mean of error distance comparison in three situations 


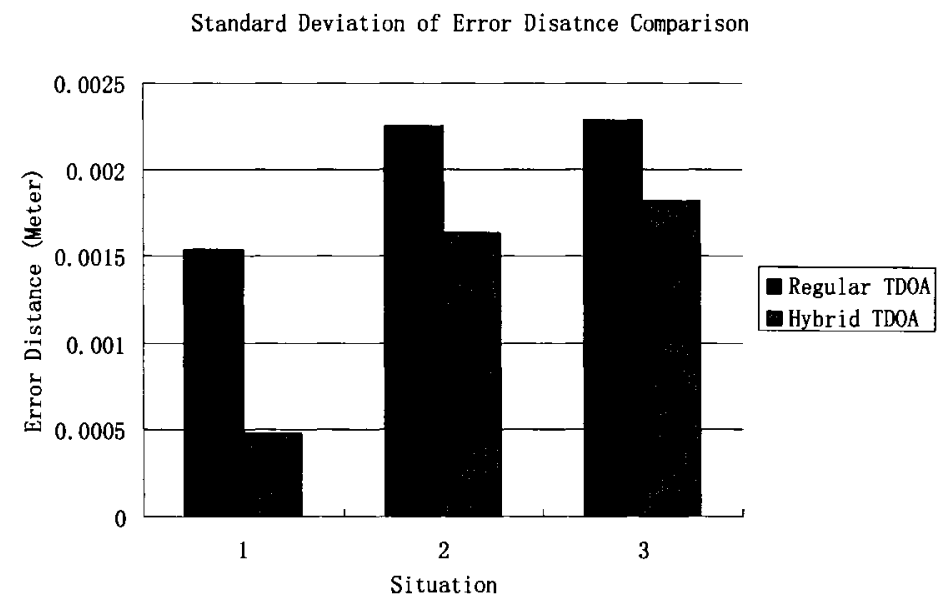

Figure 4-22 Standard deviation of error distance comparison in three situations

From the above tables and figures, the following statements can be derived:

- When the number of category No. 1 refer-triangles increases, the accuracy of TDOA measurement decreases.

- When the number of category No. 1 refer-triangles increases, the standard deviation of error distance increases. Consequently, the probability that one estimation process obtains less accurate results than the average value increases.

- In all three situations, the Hybrid TDOA method performs better than the regular LS TDOA method in both average value and standard deviation.

- For the Hybrid TDOA method, when the number of refer-triangles increases (from two or three to five), both the DisError and standard deviation of DisError decrease, as shown in all above tables and figures.

The simulation of the influence of the number of reference nodes without request on a certain topology will be shown in Section 4.4, Performance of TDOA and Hybrid TDOA 
Method in Entire Network.

\subsubsection{Influence of Noise in Estimation}

In TDOA estimation, the influence of noise on the precision of the estimation should be minor, because noise cannot change the propagation time of the packets; Noise can only corrupt a packet, making a packet unreadable in the receiver. This simulation is used to verify whether the influence of noise is minor in different topologies. In this section, the three categories of the relative positions are used again. The noise can affect whether a wireless frame can be received correctly, thus, influences the probability that a TDOA measurement will be observed.

\subsubsection{NS2 Propagation Model and Noise Environnent}

NS2 provides three propagation models-Free Space, Two-ray Ground, and Shadowing, to predict the signal power received by the receiver. The Free Space and Two-ray Ground models are employed to simulate the path loss of wireless communication when a line-of-sight path exists between the transmitter and the receiver. The first two models predict the received power as a deterministic function of distance and both represent the communication range as an ideal circle. In reality, the received power at a certain distance is a random variable, due to multi-path propagation effects (fading effects). A more general and widely-used propagation model is the Shadowing model [57]. 
To make the simulation more realistic, more parameters are introduced into the Shadowing model. All the following parameters will play a role in determining the probability that a frame will be corrupted. In [57], some typical values of path loss exponent $\beta$ and Shadowing deviation $\sigma d B$ are given, as shown in Table 4-4 and Table 4-5. A detailed introduction on this model is given in Appendix C.

Table 4-4 Environmental variable 1 in NS2 noise model

\begin{tabular}{|c|l|c|}
\hline \multicolumn{2}{|c|}{ Environment } & $\beta$ \\
\hline \multirow{2}{*}{ Outdoor } & Free Space & 2 \\
\cline { 2 - 3 } & Shadowing urban area & 2.7 to 5 \\
\hline \multirow{2}{*}{ In building } & Line-of-sight & 1.6 to 1.8 \\
\cline { 2 - 3 } & Obstructed & 4 to 6 \\
\hline
\end{tabular}

Table 4-5 Environmental variable 2 in NS2 noise model

\begin{tabular}{|l|l|}
\hline Environment & $\sigma d B(\mathrm{~dB})$ \\
\hline Outdoor & 4 to 12 \\
\hline Office, hard partition & 7 \\
\hline Office, soft partition & 9.6 \\
\hline Factory, line-of-sight & 3 to 6 \\
\hline Factory, obstructed & 6.8 \\
\hline
\end{tabular}

Those values in the two tables can be set in NS2 to determine the environmental situations for the Shadowing model.

The effect of noise in TDOA comes down to the probability that a packet is corrupted beyond recognition. This probability can be determined by setting the parameters in the Shadowing Model.

NS2 mainly employs thresholds to determine whether one frame has been received correctly by the receiver [57 to 60]. A parameter (CSThresh_) in the signal strength 
threshold determines whether one frame can be detected in the receiver. When the signal strength of the frame is less than CSThresh_, this frame will be discarded in the physical layer and will not be visible to the MAC layer. Moreover, there is another parameter (RxThresh_) in NS2 for the signal strength of one frame received by the receiver. When one frame is received and if the signal strength of the received frame is weaker than RxThresh_, the frame is tagged as a corrupted frame and will be discarded in the MAC layer. If not, this frame is received correctly. Additionally, in the situation where multi-frames are received simultaneously by a receiver, NS2 provides a third parameter (CPThresh_). When this situation occurs, the ratio of the strongest frame's signal strength to the signal strength sum of other frames is calculated. If it is less than CPThresh_, all frames will be collided and discarded. If not, the strongest frame is received correctly and other frames are ignored. Detailed information on the Shadowing model can be found in Appendix C. By setting different values for the three parameters in the Shadowing models, different noisy environments can be simulated in NS2.

In a noisy environment, the probability of a packet being correctly received at a certain distance will decrease as the noise increases. In a wireless environment, the noise includes noise generated by the receiver and noise from the environment. But the different receivers and different environments have different noise distributions. We make a certain run under a constant noise level. In other words, the probability that a packet can be recognized is set at a fixed value in one run. With the probability varying, different noisy environments can be simulated. 
In this way, The RXThresh_is used to set different noisy environments. NS2 provides a tool to calculate the RXThresh_ with the different probabilities that a packet can be correctly received at a certain distance. This simulation will employ two different environments: an outdoor urban area $(\beta=4, \sigma d B=8)$ and indoor line-of-Sight $(\beta=1.7$, $\sigma d B=4.5$ ). Those values are chosen because it has been proved that they can reflect the real world environment in [60].

Holding the assumption that the ideal communication range of a mobile node is 250 meters, Table 4-6 provides different RXThresh_parameters under different probabilities that a packet can be correctly received at 250 meters in the two different environments.

Table 4-6 Noise level definition

\begin{tabular}{|c|l|l|}
\hline Probability of receiving a packet correctly & RXThresh_(outdoor) & RXThresh_(indoor) \\
\hline 0.95 (Noise level set to 0.05) & $2.37841 \mathrm{e}-15$ & $2.93257 \mathrm{e}-9$ \\
\hline 0.85 (Noise level set to $\mathbf{0 . 1 5}$ ) & $7.29497 \mathrm{e}-15$ & $5.50855 \mathrm{e}-9$ \\
\hline 0.75 (Noise level set to $\mathbf{0 . 2 5}$ ) & $1.42095 \mathrm{e}-14$ & $8.01515 \mathrm{e}-9$ \\
\hline 0.65 (Noise level set to $\mathbf{0 . 3 5}$ ) & $2.42055 \mathrm{e}-14$ & $1.08153 \mathrm{e}-8$ \\
\hline 0.55 (Noise level set to $\mathbf{0 . 4 5}$ ) & $3.90518 \mathrm{e}-14$ & $1.41542 \mathrm{e}-8$ \\
\hline 0.45 (Noise level set to $\mathbf{0 . 5 5}$ ) & $6.20439 \mathrm{e}-14$ & $1.83645 \mathrm{e}-8$ \\
\hline 0.35 (Noise level set to $\mathbf{0 . 6 5}$ ) & $1.00098 \mathrm{e}-13$ & $2.4034 \mathrm{e}-8$ \\
\hline 0.25 (Noise level set to $\mathbf{0 . 7 5}$ ) & $1.70515 \mathrm{e}-13$ & $3.24304 \mathrm{e}-8$ \\
\hline 0.15 (Noise level set to $\mathbf{0 . 8 5}$ ) & $3.32137 \mathrm{e}-13$ & $4.71875 \mathrm{e}-8$ \\
\hline 0.05 (Noise level set to $\mathbf{0 . 9 5}$ ) & $1.01872 \mathrm{e}-12$ & $8.86373 \mathrm{e}-8$ \\
\hline
\end{tabular}

In the following runs, about 2,000 location identification requests are issued from the source node. The average value of those successful TDOA estimations is obtained and the percentage of successful estimations is also obtained.

\section{Category No. 1}

For category No. 1, the following charts show the result. 
Noise VS. Estimation accuracy

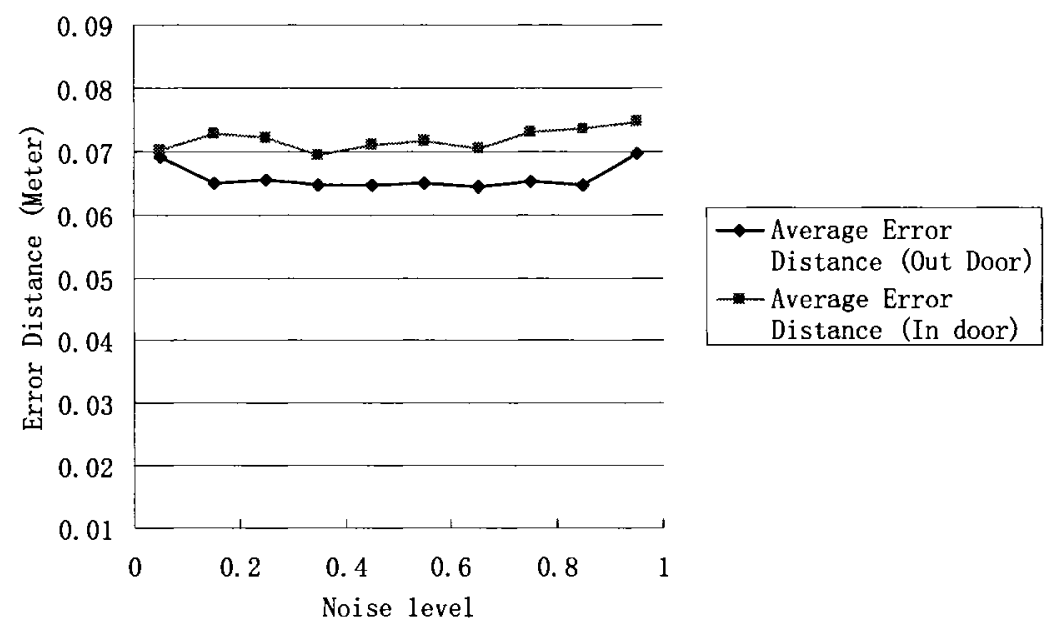

Figure 4-23 Accuracy vs. Noise Category No. 1

Noise VS. Success TDOA measurement

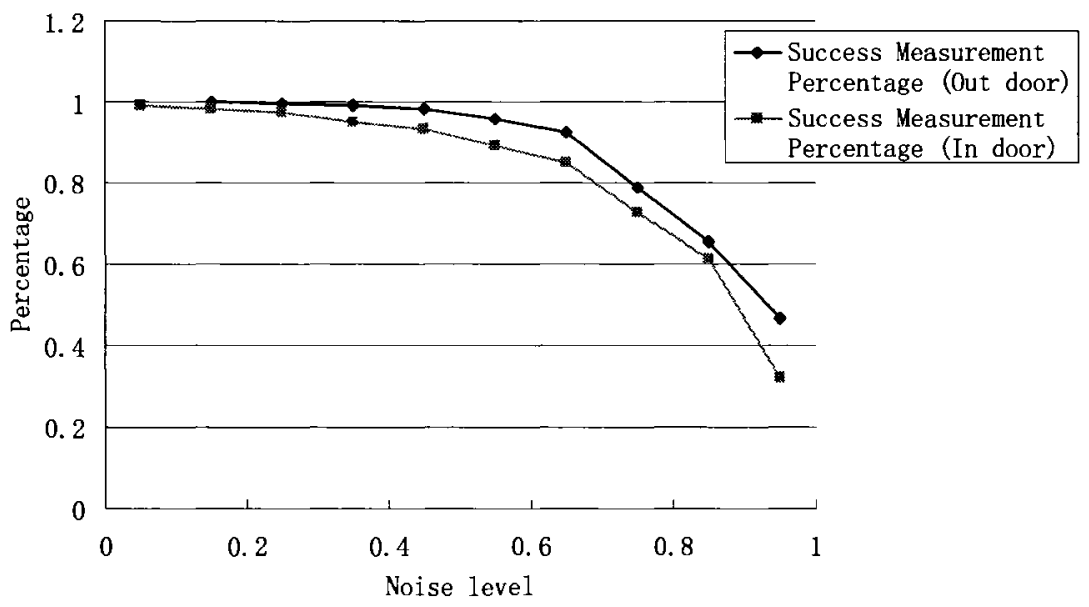

Figure 4-24 Successful estimation percentage vs. Noise Category No. 1

From Figure 4-23 and Figure 4-24, it is clear that the influence of noise on TDOA estimation accuracy is not very great, but when the noise level increases, some TDOA measurements cannot be done because some of the reference nodes cannot receive the location request frame correctly.

For indoor and outdoor situations, the same results are obtained from Figure 4-23 and 
Figure 4-24. In order to reduce the complexity of the simulation, the other two categories will only run outdoor scenarios.

\section{Category No. 2}

Figure 4-25 and Figure 4-26 are obtained from the simulation.

Noise VS. Estimation accuracy

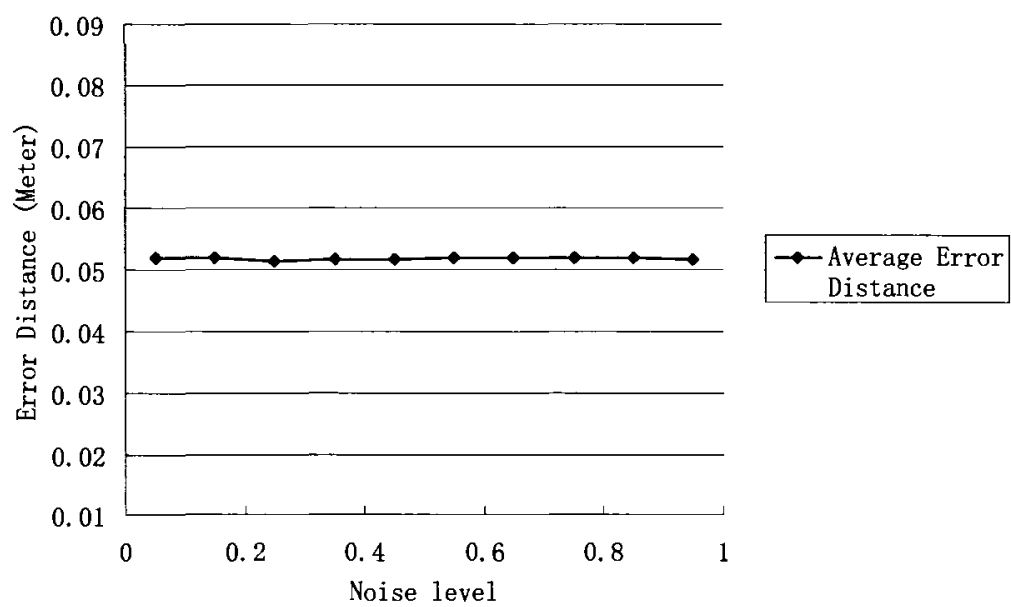

Figure 4-25 Accuracy vs. Noise Category No. 2

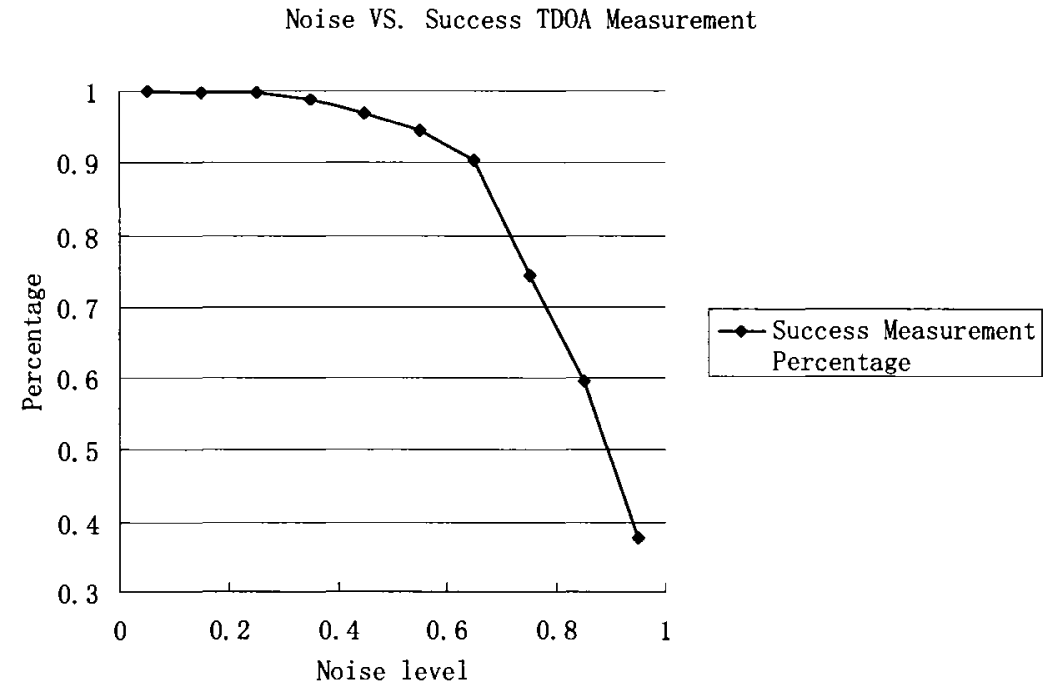

Figure 4-26 Successful estimation percentage vs. Noise Category No. 2

For category No. 2, the TDOA estimation accuracy is around 0.062 meters in an 
outdoor situation and the accuracy stays almost constant at different noise levels. The percentage of success measurement decreases from $1 \%$ to around $35 \%$ when the noise level increases.

\section{Category No. 3}

Figure 4-27 and Figure 4-28 are obtained from the simulation of category No. 3 in outdoor situations.

Noise VS. Average Error Distance

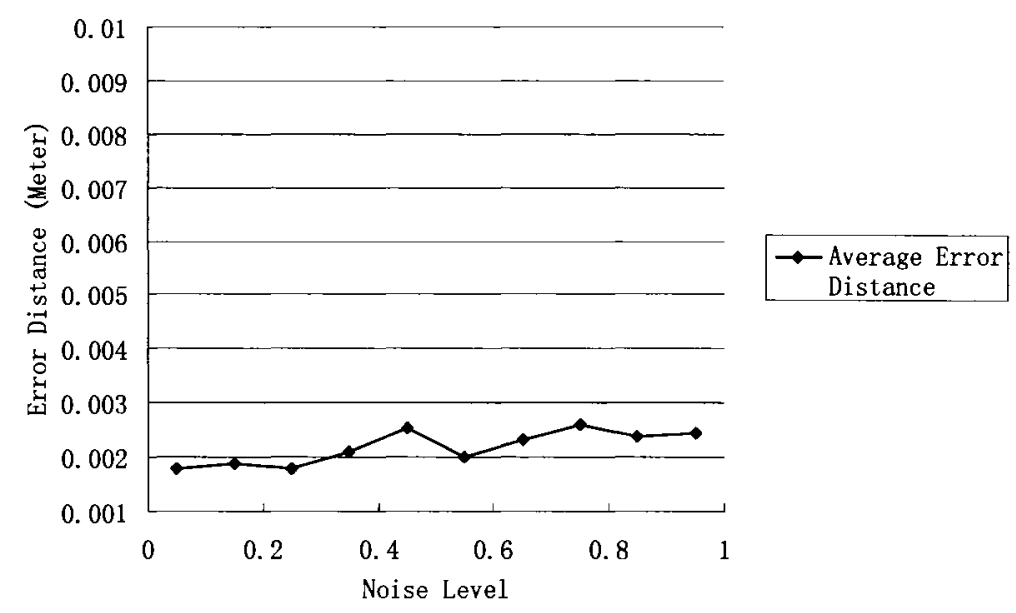

Figure 4-27 Accuracy vs. Noise Category No. 3

Noise VS. Success TD0A measurement

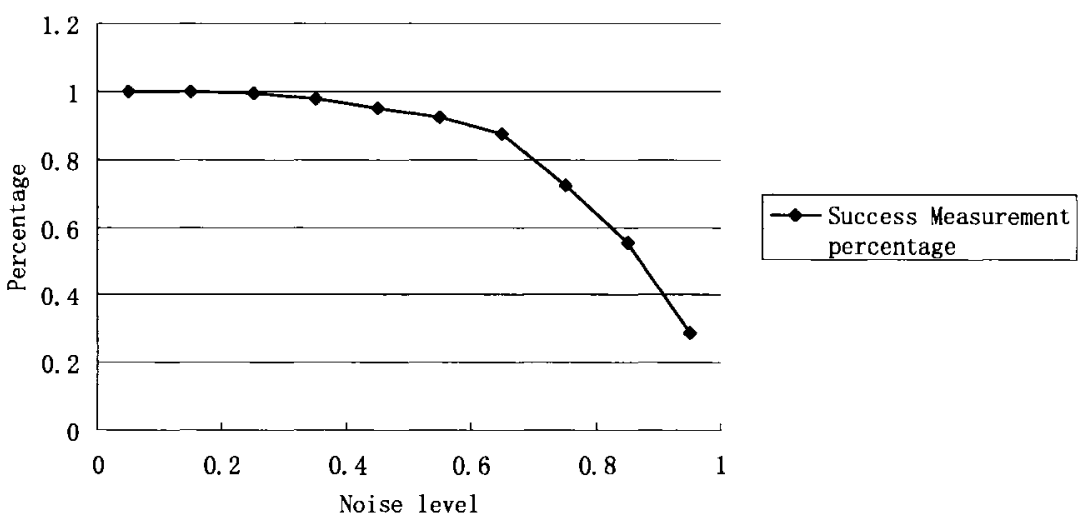

Figure 4-28 Successful estimation percentage vs. Noise Category No. 3 
Category No. 3 obtained the highest estimation accuracy around 0.002 meters and the accuracy stayed almost constant at different noise levels. However, the percentage of success measurement decreases from $1 \%$ to $30 \%$ when the noise level increases.

A comparison of the percentages of success measurement between the three categories in outdoor situations is made as shown in the following charts.

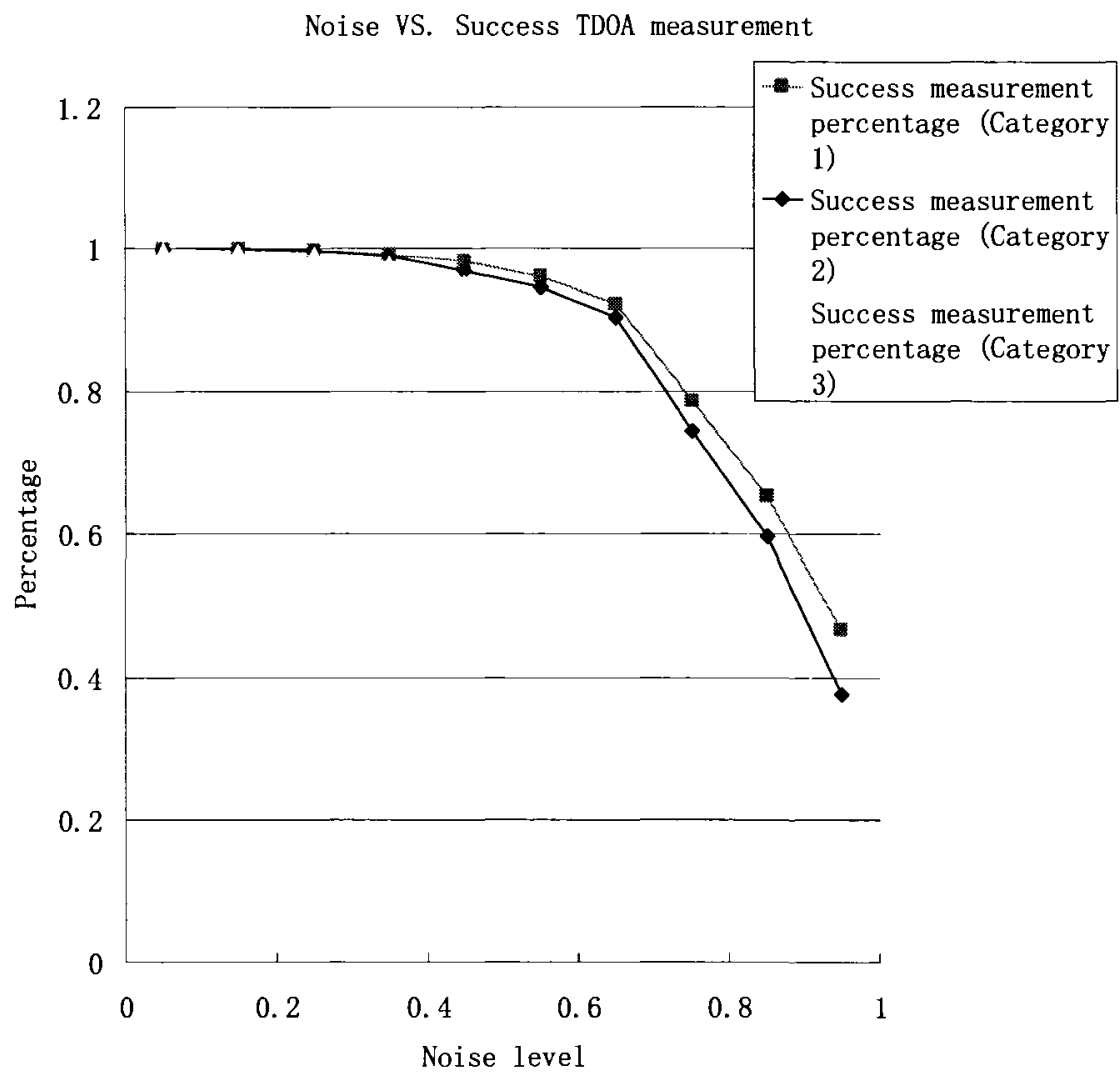

Figure 4-29 Percentage of success estimation vs. noise level

Figure 4-29 shows that category No. 3 has the lowest resistance to noise. Category No. 2 gets better. Category No. 1 is the best when it comes to noise. To sum up, however, the noise influence on TDOA accuracy estimation is minor.

As Figure 4-29 and other figures in this section show, noise level greatly influences 
whether a reference node can successfully receive a signal from a source node, which, in turn, can influence how many reference nodes can be used in a certain location estimation process. Thus, the noise level can also influence accuracy, when $M$ is greater than 3. A detailed discussion on noise level vs. accuracy, when $M$ is greater than 3, will be given in Section 4.4.5.

\subsection{Performance of TDOA and Hybrid TDOA Method in Entire Network}

This section does not focus on a certain estimation process. This section verifies the long-term performance of TDOA and Hybrid TDOA in a uniformly distributed mobile Ad Hoc/sensor network under different conditions (number of reference nodes, density of reference nodes, and noise level). Because this whole section will deal with uniformly distributed and uniformly moving nodes, the batch length, initialization bias and the confidence interval of the simulation are discussed.

\subsubsection{Initialization Bias and Batch Length}

All nodes in the Ad Hoc/Sensor network in this simulation are uniformly distributed and moving. Compared with Section 4.3, the initialization bias and batch length should be considered first. The initialization bias is the bias in the point estimator due to artificial or arbitrary initial conditions. The bias can be severe if the run length is too short, but it generally decreases as the run length increases [61]. In Section 4.3.1, 100 TDOA 
estimations were simulated and the result shows that, in the worst case, 20 estimations can cover at least one exception point of estimations. In the following long-term runs, it is reasonable to set the batch length to 30 estimation processes (slightly larger than the smallest possible batch length), which statistically allows each batch sample to have different estimations of a source node in this Ad Hoc/Sensor network simulation.

It is now possible to obtain the initialization bias.

The initialization bias check setting is as follows:

Simulation area $=[1000 \mathrm{~m}, 1000 \mathrm{~m}]$

Environment $=$ Outdoor

Noise level $=0.95$ (reflecting the worst environment)

Reference node number $=40$

Batch length $=30$

Total measurement $=3000$

Location identification method $=$ Regular LS TDOA

Replication time $=4$

The simulation result is shown in Figure 4-30: 
Initial bias

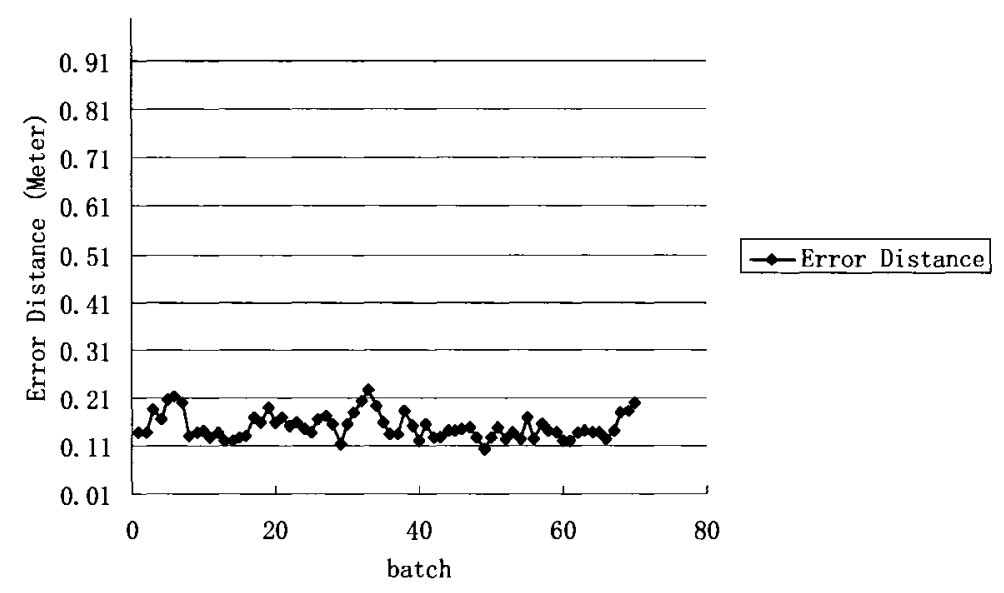

Initial bias

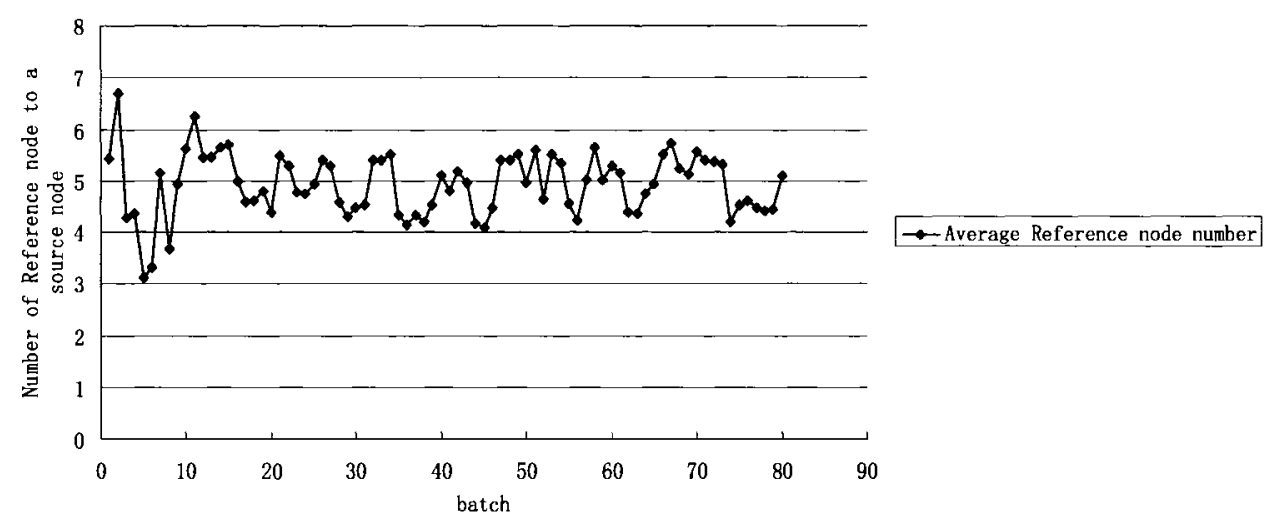

Figure 4-30 Initial bias of regular LS TDOA

The first figure in Figure 4-30 shows the average DisError in this simulation. From this figure, there is no clear initial bias. The second figure shows the average number of reference nodes for a source node, which shows that after batch 15 , the average number of reference nodes for a source node is in stable status, so the bias should be 15 batches.

Another initialization bias check that is made based on the average number of reference nodes to a source node is much bigger than the previous one. The following is 
the second initialization bias check setting:

The simulation result is given by the following figures:

Simulation area $=[1000 \mathrm{~m}, 1000 \mathrm{~m}]$

Environment $=$ Outdoor

Noise level $=0.95$ (reflecting the worst environment)

Reference node number $=16$

Batch length $=30$

Total measurement $=3000$

Location identification method $=$ Regular LS TDOA

Replication time $=4$

The simulation result is shown in Figure 4-31: 
Initial bias

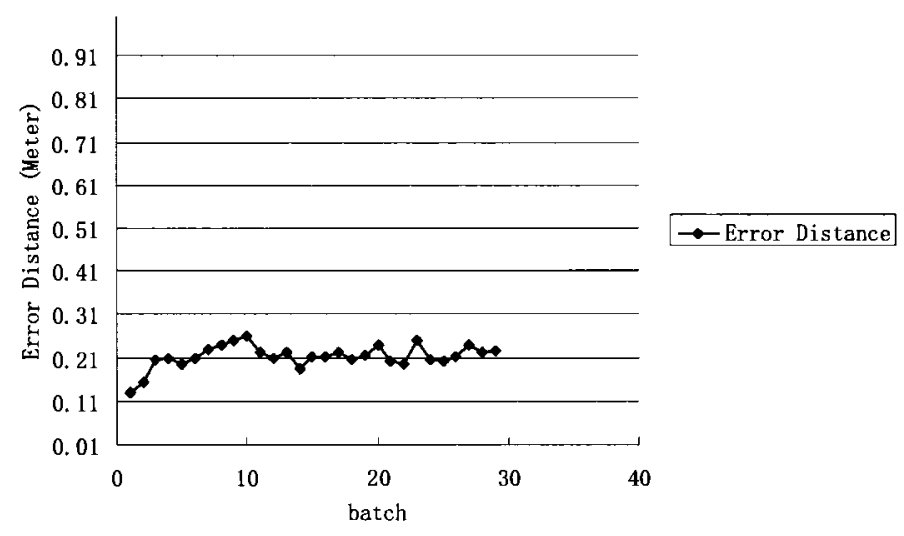

Initial bias

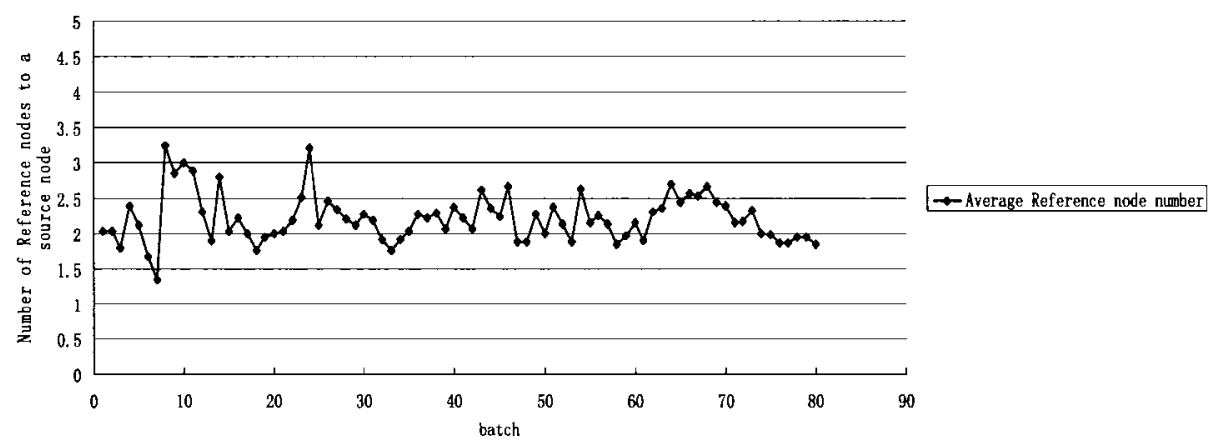

Figure 4-31 Initial bias of Hybrid TDOA

The first figure in Figure 4-31 shows that the average DisError is in stable status after batch 6 in this simulation. From that figure, only 29 batches are obtained, because in this scenario, sometimes, there are less than three reference nodes around a source node (this can be proven from the second figure, which shows that the average number of reference nodes to a source node is around 2), which makes a TDOA estimation impossible. Such situations are ignored; only successful TDOA estimations are counted. The second figure shows that after batch 25 (this corresponds to batch 9 in the first figure), the average number of reference nodes for a source node is in stable status. This bias length is bigger than the first check. Hence, in the following simulations, 25 batches are taken as the 
initialization bias and the data collected during the first 25 batches of measurements will be ignored.

\subsubsection{Confidence Interval}

In this section, the confidence intervals of the two former scenarios are tested and set.

The confidence interval test is used to evaluate whether the simulation and the realistic system are close enough [61]. Considering that the DisError is the major concern in the simulation, the following process focuses on the simulation values of DisError.

From [61], if $Y$ is the simulation output and $\mu=E(Y)$, the confidence interval for $\mu$ is given by:

$$
\bar{Y} \pm t_{\alpha / 2, n-1} S / \sqrt{n}
$$

where $S^{2}=\frac{\sum_{i=1}^{n}\left(Y_{i}-\hat{\theta}\right)^{2}}{(n-1) n}$

$Y_{i}$ is the average value of replication $i^{\text {th }}$. And $\hat{\theta}$ is the average value of all replications. In this simulation, the requirement is that the connectivity probability is within $\pm 0.02(\varepsilon=0.02)$ of the estimated value with a probability of $95 \%$. We then get $\alpha=(1-95 \%)=0.05$.

For the 16-reference-node scenario, the following table shows the error distance data collected in that simulation, in which the first 10 batches are ignored as initialization bias. 
Table 4-7 Confidence interval data table

\begin{tabular}{|c|r|r|r|r|r|}
\hline Batch & \multicolumn{1}{|c|}{ R1 } & \multicolumn{1}{c|}{ R2 } & \multicolumn{1}{c|}{ R3 } & \multicolumn{1}{c|}{ R4 } & \multicolumn{1}{c|}{ Average } \\
\hline 11 & 0.24296 & 0.189325 & 0.252648 & 0.199602 & 0.22113375 \\
\hline 12 & 0.233228 & 0.19736 & 0.220294 & 0.188783 & 0.20991625 \\
\hline 13 & 0.182079 & 0.272106 & 0.197228 & 0.232428 & 0.22096025 \\
\hline 14 & 0.267059 & 0.159313 & 0.187646 & 0.127916 & 0.1854835 \\
\hline 15 & 0.275398 & 0.167851 & 0.268078 & 0.142324 & 0.21341275 \\
\hline 16 & 0.196249 & 0.251542 & 0.2603 & 0.135801 & 0.210973 \\
\hline 17 & 0.253255 & 0.189325 & 0.250821 & 0.20254 & 0.22398525 \\
\hline 18 & 0.235862 & 0.19736 & 0.215209 & 0.172741 & 0.205293 \\
\hline 19 & 0.217832 & 0.172106 & 0.222142 & 0.252698 & 0.2161945 \\
\hline 20 & 0.198058 & 0.235155 & 0.244031 & 0.286711 & 0.24098875 \\
\hline 21 & 0.156856 & 0.218507 & 0.235034 & 0.196356 & 0.20168825 \\
\hline 22 & 0.159313 & 0.167969 & 0.228697 & 0.229432 & 0.19635275 \\
\hline 23 & 0.267851 & 0.24296 & 0.212023 & 0.273092 & 0.2489815 \\
\hline 24 & 0.166495 & 0.233228 & 0.214889 & 0.200512 & 0.203781 \\
\hline 25 & 0.17256 & 0.182079 & 0.296598 & 0.152301 & 0.2008845 \\
\hline 26 & 0.285063 & 0.167059 & 0.181007 & 0.21395 & 0.21176975 \\
\hline 27 & 0.311561 & 0.275398 & 0.21294 & 0.154258 & 0.23853925 \\
\hline 28 & 0.27186 & 0.159313 & 0.268702 & 0.184129 & 0.221001 \\
\hline 29 & 0.256233 & 0.167851 & 0.296539 & 0.179899 & 0.2251305 \\
\hline Average & 0.217039517 & 0.197109103 & 0.227465586 & 0.210564621 & 0.213044707 \\
\hline & & & & & \\
\hline
\end{tabular}

From Table 4-7, the following results can be obtained:

$$
\begin{aligned}
& \hat{\theta}=0.213413 \\
& S=0.006351 \\
& T_{\alpha / 2, n-1} S=T_{0.025,3} \times 0.006351=0.0202
\end{aligned}
$$

NOTE: $T_{0.025,3}$ can be found in [27], Appendix table A.5.

The confidence interval is $[0.213413-0.0202,0.213413+0.0202]=[0.193213$,

\section{$0.233613]$.}

It is now possible to consider how many replications are needed in this simulation to cater to the former requirement. From the requirement, we get the following expression: 


$$
P[|\hat{\theta}-\theta|<0.0213413] \geq 0.95
$$

From [6], we get the minimum necessary replications $n$ as follows, where $(\varepsilon=0.002)$ :

\begin{tabular}{|c|l|c|}
\hline $\boldsymbol{n}$ & $\mathbf{2}$ & $\mathbf{3}$ \\
\hline $\mathrm{T}_{\alpha / 2 \mathrm{n}-1}$ & 12.71 & 4.3 \\
\hline $\begin{array}{c}\mathrm{T} \\
\mathrm{n}-1 \mathrm{~S} / \varepsilon)^{2}\end{array}$ & 16.29 & 1.86 \\
\hline
\end{tabular}

From the values in the previous table, three replications are enough to guarantee that the connectivity probability is within $\pm 0.02(\varepsilon=0.02)$ of the estimated value with a probability of $95 \%$.

For the 40-reference-node scenario, the same process is executed.

$$
\begin{aligned}
& \hat{\theta}=0.198418 \\
& S=0.00593397 \\
& T_{\alpha / 2, n-1} S=T_{0.025,3} \times 0.00593397=0.01887
\end{aligned}
$$

The confidence interval is $[0.198418-0.01887,0.198418+0.01887]=[0.188983$, $0.207853]$.

The following table is then obtained, where $(\varepsilon=0.02)$.

\begin{tabular}{|l|l|l|}
\hline$n$ & 2 & 3 \\
\hline $\mathrm{T}_{\alpha / 2 \mathrm{n}-1}$ & 12.71 & 4.3 \\
\hline$\left(\mathrm{T}_{\alpha / 2 \mathrm{n}-1} \mathrm{~S} / \varepsilon\right)^{2}$ & 37.35 & 1.62 \\
\hline
\end{tabular}

From the values in the above table, three replications are enough to guarantee that the connectivity probability is within $\pm 0.02(\varepsilon=0.02)$ of the estimated value with a probability of $95 \%$. 


\subsubsection{Performance vs. Number of Reference Nodes}

This section verifies the influence of number of reference nodes for the regular least square TDOA and Hybrid TDOA methods. In this section, only one source node and $N$ reference nodes are uniformly distributed and uniformly moving in the simulation area. DisError is measured over the long term, and the number of reference nodes that can be used in one location estimation process will be increased from three to nine.

Obviously, in the Hybrid TDOA method, some refer-triangles may be isolated from the TDOA estimation process in some situations. For the regular least square TDOA method, all reference nodes that can be reached from the source node will contribute to the location estimation. After the simulation has started, the source node may have a different number of reference nodes. Only the situation in which the source node has $M$ $(M=3,4,5 \ldots)$ is traced. For example, in one run, the four reference node situation is tested; other situations in which the source node has more or less than four reference nodes will be ignored.

The environment settings are as follows:

Simulation area $=[1000 \mathrm{~m}, 1000 \mathrm{~m}]$

Environment $=$ Outdoor

Noise level $=0.95$ (reflecting the worst environment)

Reference node number around the source node $=M$

Batch length $=30$ 
Total estimation $=3000$

Location identification method $=$ Regular LS TDOA and Hybrid TDOA

Replication time $=3$

In one run, only those estimations in which the source node has $M$ ( $M \in\{3,4,5,6,7,8,9\})$ reference nodes are measured, so that with $M$ increase from three to nine, the average performance of the regular least square TDOA and Hybrid TDOA methods can be measured. Figure 4-32 shows the simulation result.

Long term performance Against Number of reference nodes to a source node

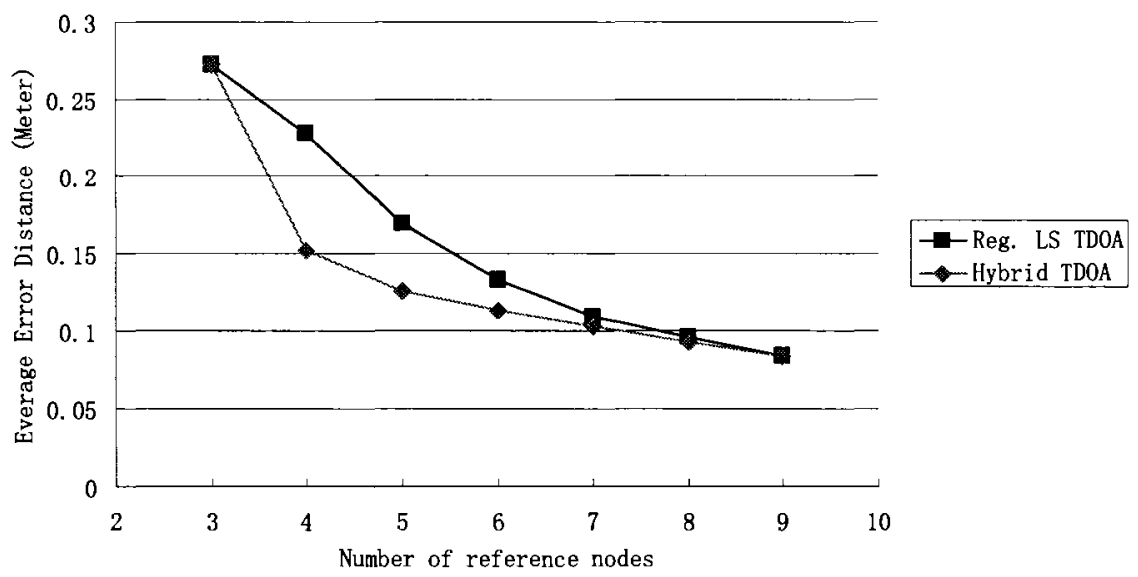

Figure 4-32 Long term performance of Reg. LS TDOA vs. Hybrid TDOA

Figure 4-32 shows that when the number of reference nodes increases from four to seven, the Hybrid TDOA method performs better than the regular least square TDOA method. Especially when the number of reference nodes is four and five, the performance of the Hybrid TDOA method is much better than the regular least square TDOA method. However, the DisError gap between the Hybrid TDOA and the regular least square TDOA method decreases after six; moreover, the performance of the Hybrid TDOA and 
the regular least square TDOA is almost the same when the number of reference nodes is nine. Why does that happen?

Figure 4-33 shows the percentage of Category No. 1 combinations in all possible combinations of the TDOA method, when the number of reference nodes is greater than three.

Category No. 1 in all combinations

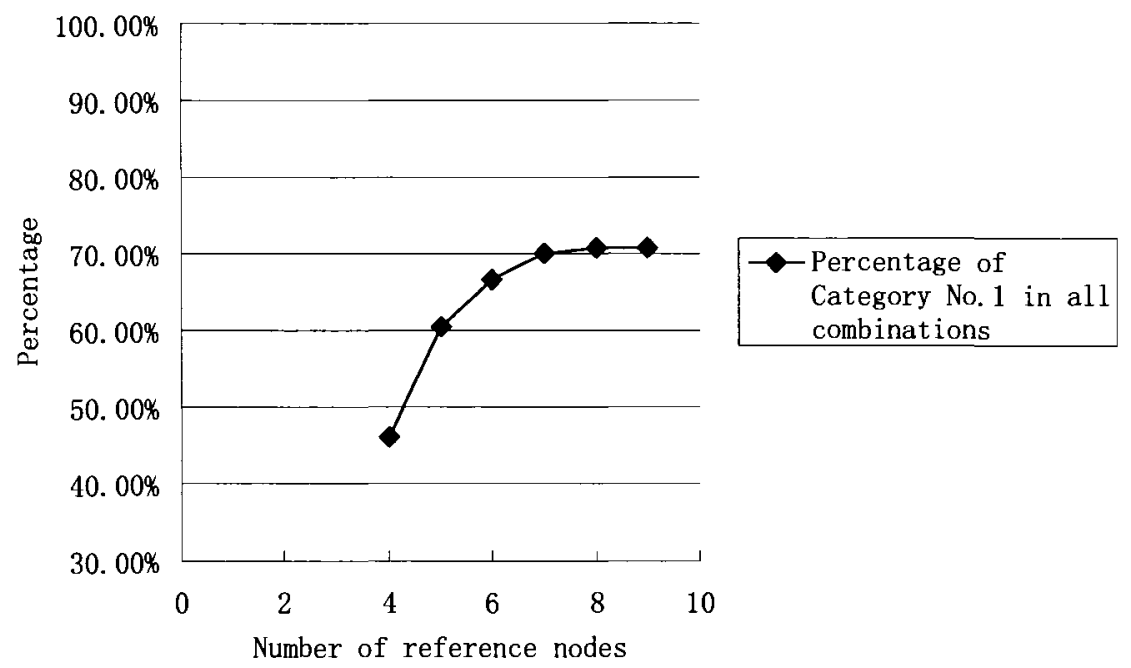

Figure 4-33 Category No. 1 in all possible combinations

Figure 4-33 clearly shows that from four to six, the percentage of Category No. 1 in all combinations increases greatly, which can explain why the Hybrid TDOA method performs much better than the regular least square TDOA method. It is still unknown why the performance gap between them becomes smaller and smaller once the number of reference nodes is greater than seven, although the percentage of Category No. 1 increases very little once the number of reference nodes is greater than seven, which is still larger than the percentage of category No. 1 when the number of reference nodes is 
smaller than seven. Table 4-8 provides more clues on that.

Table 4-8 Average category No. 1 in different number of reference nodes

\begin{tabular}{|c|l|l|l|}
\hline $\begin{array}{l}\text { Number of Reference } \\
\text { Nodes }\end{array}$ & $\begin{array}{l}\text { Total Number of } \\
\text { Combinations }\end{array}$ & $\begin{array}{l}\text { Category No.1 } \\
\text { (Average) }\end{array}$ & Others (Average) \\
\hline 4 & $c_{4}^{3}=4$ & 1.84 & 2.16 \\
\hline 5 & $c_{5}^{3}=10$ & 6.05 & 3.95 \\
\hline 6 & $c_{6}^{3}=20$ & 13.32 & 6.68 \\
\hline 7 & $c_{7}^{3}=35$ & 24.49 & 10.51 \\
\hline $\mathbf{8}$ & $c_{8}^{3}=56$ & 39.65 & 16.35 \\
\hline 9 & $c_{9}^{3}=84$ & 59.36 & 24.64 \\
\hline
\end{tabular}

Table 4-8 shows the number of category No. 1 increases from an average of 1.84 to 59.36. Although the accuracy of category No. 1 is worse than categories No. 2 and No. 3, more TDOA measurements of category No. 1 can also increase the accuracy of category No. 1 measurements themselves. Moreover, Table 4-8 clearly shows that the number of category No. 1 increases faster than other categories, making the accuracy of category No.1 measurement increase faster than the other categories, because more category No. 1 TDOA measurements increase the estimation accuracy of all category No.1 refer-triangles. That is why the performance gap between hybrid TDOA and regular TDOA becomes smaller when the number of reference nodes is greater than seven.

\subsubsection{Performance vs. Density of Reference Nodes}

With the increase of reference-node density, the probability that a source node will 
reach more reference nodes increases. This section measures the influence of the reference-node density on the performance of the regular least square TDOA and Hybrid TDOA methods. In this simulation, $N$ source nodes and $M$ reference nodes are uniformly distributed and move uniformly in the simulation area, in which $N$ is far smaller than $M$.

The environment settings are as follows:

Simulation area $=[1000 \mathrm{~m}, 1000 \mathrm{~m}]$

Environment $=$ Outdoor

Noise level $=0.95$ (reflecting the worst environment)

Reference node number around the source node $=M$

Batch length $=30$

Total measurement $=3000$

Location identification method $=$ regular LS TDOA and Hybrid TDOA

Replication time $=3$

Considering the simulation area is fixed at $[1000 \mathrm{~m}, 1000 \mathrm{~m}]$, the density of the reference nodes is decided by the number of reference nodes in the area. In this scenario, the number of reference nodes $(M)$ will increase from 16, 20, 30, 40 to 50 in turn. Figure 4-34 shows the result. 
Long term performance against density of reference nodes

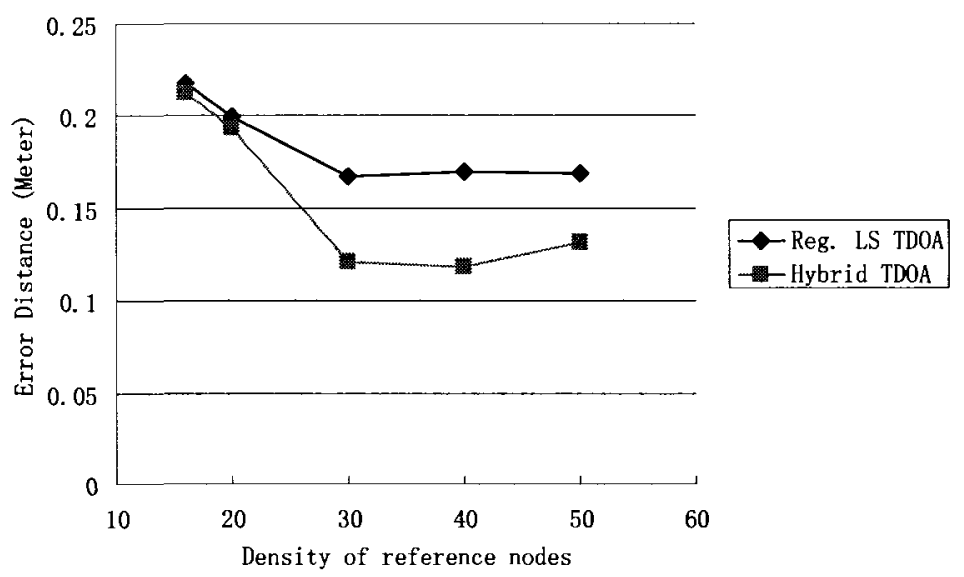

Figure 4-34 Long-term performance vs. density of reference nodes

From Figure 4-34, the DisError does not decrease after the density of reference nodes (num of nodes $/ \mathrm{km}^{2}$ ) is beyond 30, whichever the regular least square TDOA method or Hybrid TDOA method is used. In a theoretical analysis, DisError should keep decreasing as the density of the reference nodes increases. The reason why that occurred in Figure 4-34 can be explained by Figure 4-35, which records the real average number of reference nodes that are reached by a source node in this simulation.

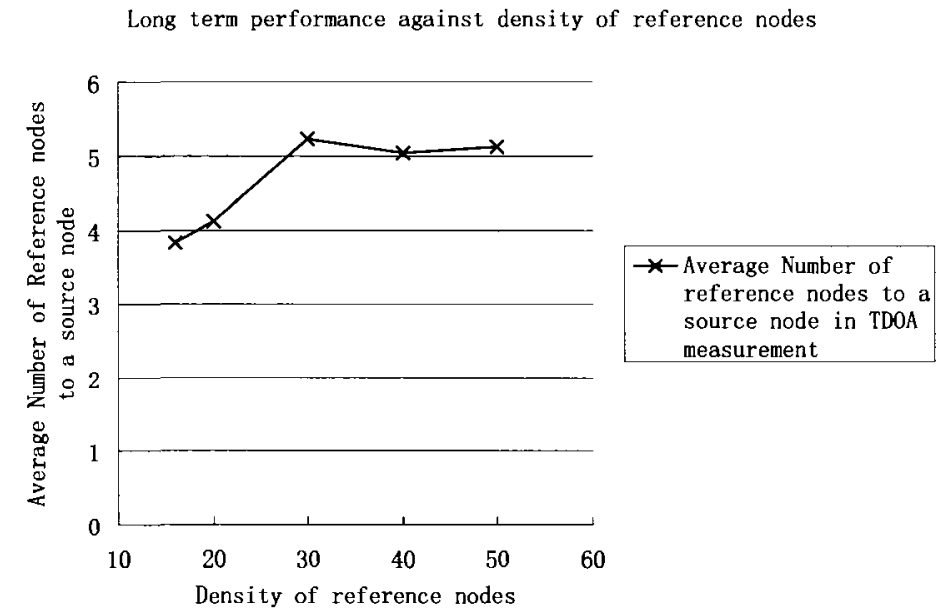

Figure 4-35 Average number of reference nodes vs. density of reference nodes 
Figure 4-35 clearly shows that after the density of reference nodes has gone over 30 , the number of reference nodes that a source node can reach does not keep increasing when the density of reference node keeps increasing, which means that in the specific physical conditions of this simulation, the real number of reference nodes for a source node, in fact, cannot increase unlimitedly. From Figure 4-35, the limit of the number of reference nodes that a source node can reach is around five. Figure 4-35 also explains why the error distance cannot decrease when the density of the reference nodes keeps increasing beyond 30 .

\subsubsection{Performance vs. Density of Reference Node \& Noise Level}

In different noise level environments, the Hybrid TDOA method shows a different average number of reference nodes that can be reached by a source node, as reflected in Section 4.3.3, which, in turn, influences the location estimation accuracy. Moreover, the density of the reference nodes also influences the location estimation accuracy in the Hybrid TDOA method and the regular least square TDOA method, as described in Section 4.4.4. Thus, the noise level and the density of the reference nodes can both influence location estimation accuracy.

In the practical location estimation service, the accuracy of the estimation is critical. This section will simulate the performance of the Hybrid TDOA method and the regular LS TDOA method with different reference node densities and different noise level environments, in which a fixed accuracy level of location estimation is required. 
Now we will look at a concrete example in order to gain a sense of the ideas expressed in this section. In a certain location estimation service system, a requirement for location estimation accuracy is the probability that the DisError is smaller than 0.2 meters should be greater than $90 \%$. In certain noisy environments, what density of reference node can satisfy this requirement? In this section, the simulation will show the percentage of location estimations in certain accuracy requirements under different reference node densities and different noise level environments, which can answer such questions.

In the simulation discussed in this section, two accuracy requirements, under 0.125 meters and 0.175 meters, are employed. Those two values are the middle values in Figure 4-32 (Section 4.4.3) and are also attained in Figure 4-34 (Section 4.4.4), which is the worst noise environment replicated in our simulations. Thus, the two values can provide enough data to analyze the influence of the noise level and the density of the reference node on the location estimation accuracy.

In this section, the noise level still includes 10 levels, as described in Table 4-6, and the density of the reference node still follows the values $(M=16,20,30,40,50,60)$, described in Section 4.4.4.

The environment settings are as follows:

Simulation area $=[1000 \mathrm{~m}, 1000 \mathrm{~m}]$

Environment $=$ Outdoor

Reference node number around the source node $=M$ 
Batch length $=30$

Total measurement $=3000$

Location identification method $=$ Regular LS TDOA and Hybrid TDOA

Replication time $=3$

The following figures, Figure 4-36 to Figure 4-39, show the performances of the regular least square TDOA method and the Hybrid method.

In following 3-D figures, $\mathrm{X}$ axis stands for the noise level, $\mathrm{Y}$ axis indicates the density of reference nodes, and $\mathrm{Z}$ axis represents the percentage of estimations that can be accepted according to the accuracy requirement.

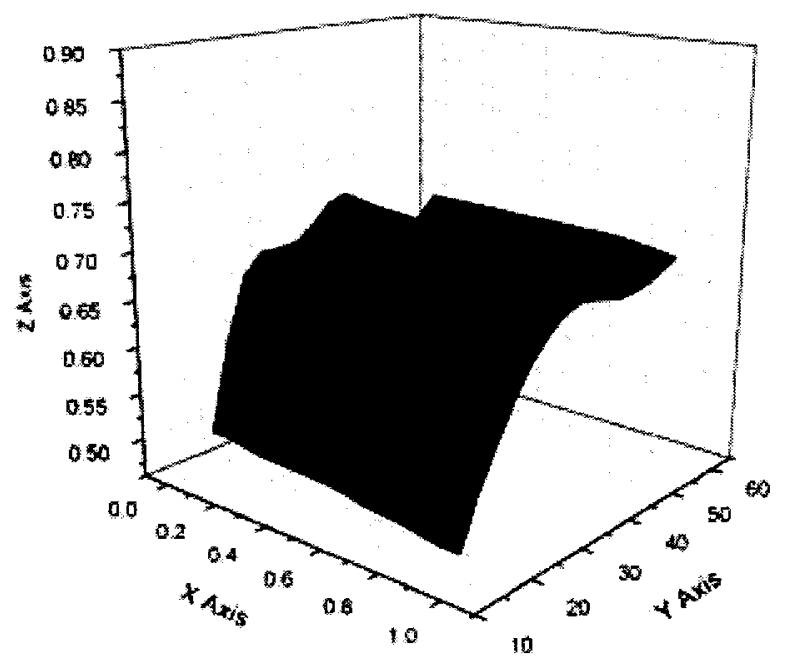

Figure 4-36 Reg. LS TDOA results (less than 0.125 meters) 


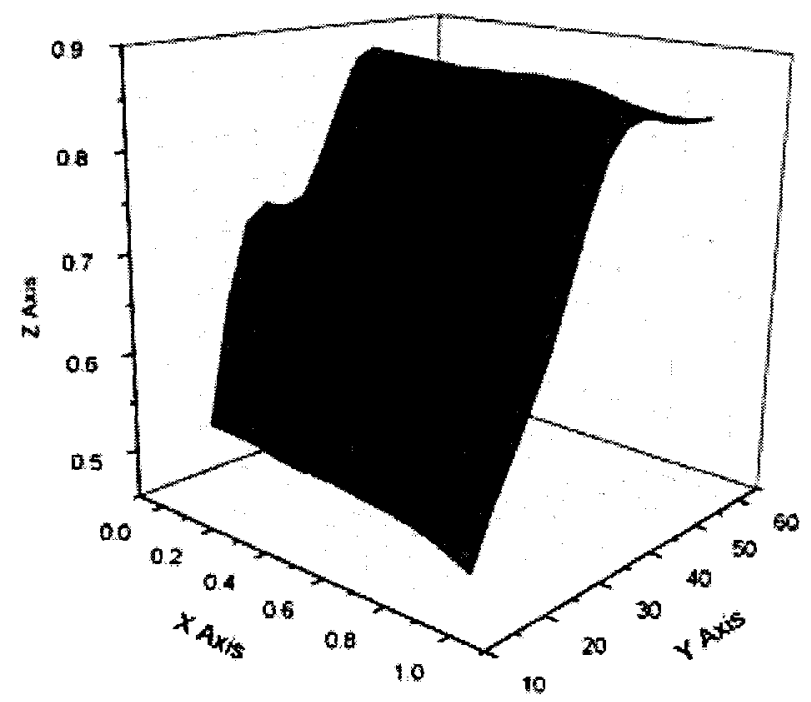

Figure 4-37 Hybrid TDOA results (less than 0.125 meters) 

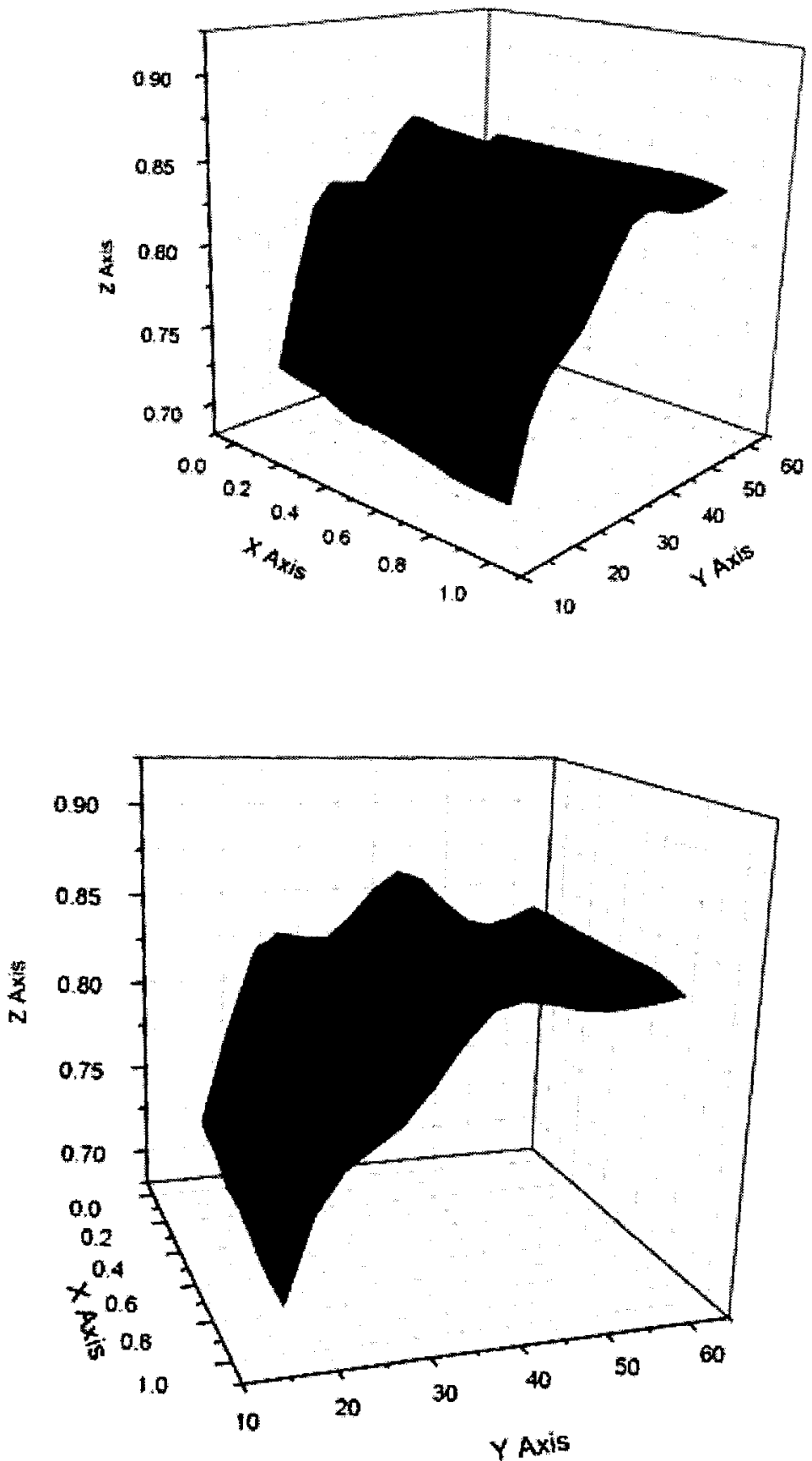

Figure 4-38 Reg. LS TDOA results (less than 0.175 meters) 

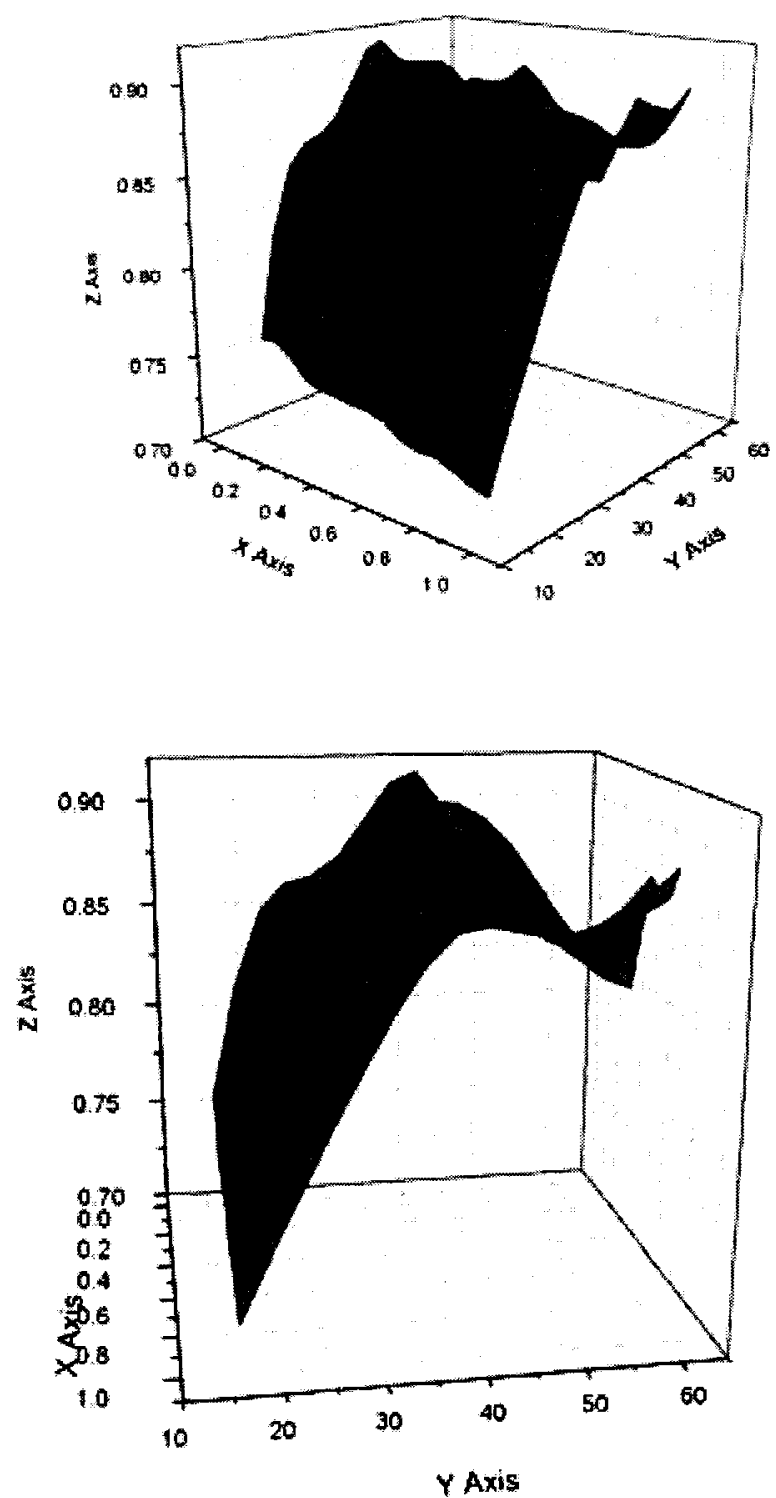

Figure 4-39 Hybrid TDOA results (less than 0.175 meters)

Those four figures clearly show that the Hybrid TDOA method performs better than the regular least square TDOA method. Moreover, those figures also show that the performance cannot increase after the density increase has gone beyond 40 , which is explained in Section 4.4.4. However, looking at those three-dimensional figures, understanding and analyzing the simulation results in detail is difficult. 
In order to make the results more understandable, Figure 4-36 to Figure 4-39 are projected onto their $\mathrm{YZ}$ planes and $\mathrm{XZ}$ planes. The new figures are shown below.
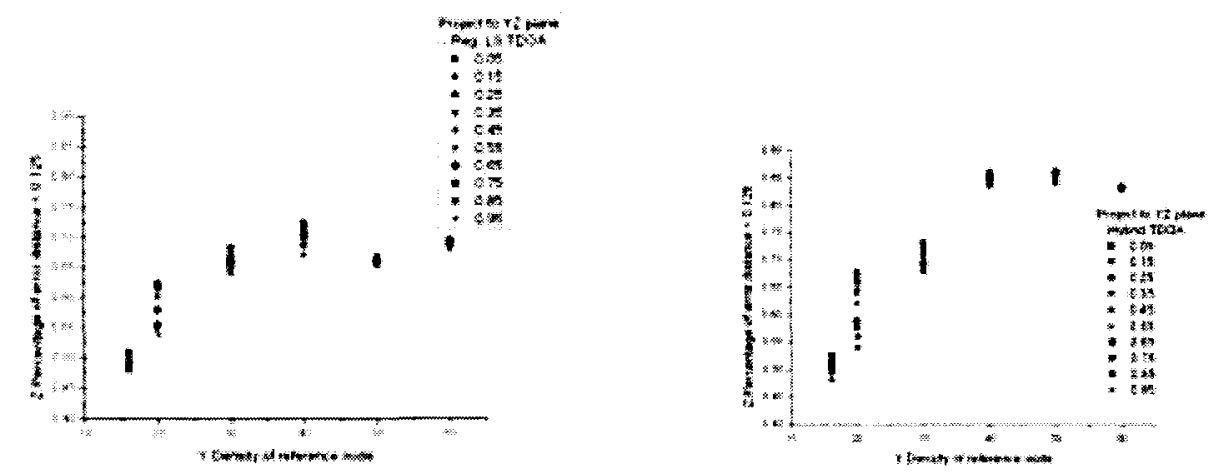

Figure 4-40 Results projected onto YZ plane (less than 0.125 meters)
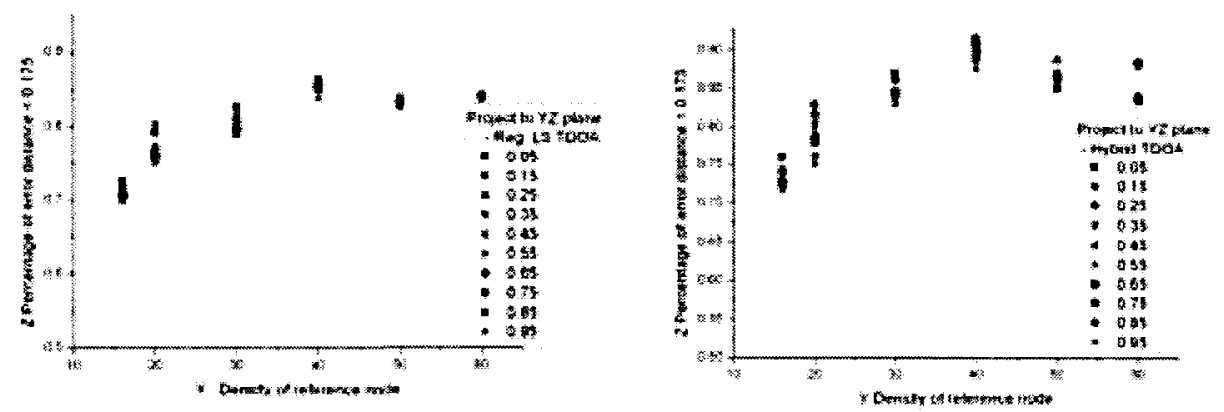

Figure 4-41 Results projected onto YZ plane (less than 0.175 meters)

In Figure 4-40 and Figure 4-41, we projected the three-dimensional figures onto the YZ plane so the influence of noise can be analyzed. It is clear that, for all densities, the performances of the regular LS TDOA method and the Hybrid TDOA method decrease, when noise level increases. Moreover, the noise influence at densities of 20 and 30 is greater than its influence at other densities. As described in Section 4.4 .4 and shown in Figure 4-35, when the density of the reference node is very large, the average number of 
reference nodes that a source node can reach in one location estimation becomes almost constant, because of the congestions between mobile nodes, which directly influence the location estimation accuracy. From Figure 4-40 and Figure 4-41, the noise level cannot affect the performance to a great extent. Also from Figure 4-40 and Figure 4-41, we see that, as the density increases, the percentage of location estimations under the accepted accuracy level increases. But once the density has become very large (more than 40 ), there was a big difference in terms of accuracy in both the Hybrid TDOA and regular LS TDOA methods, which is explained in Section 4.4.4 and Figure 4-35.
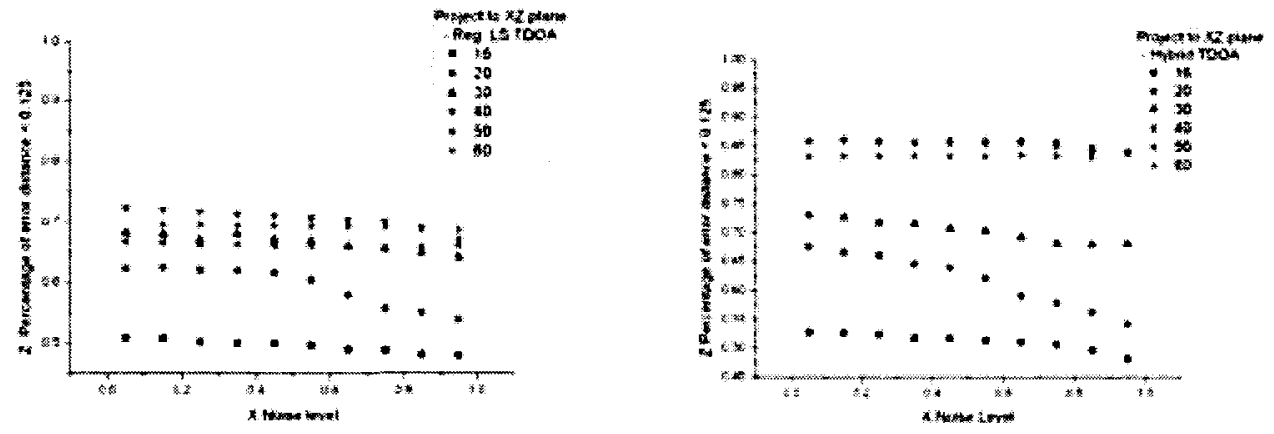

Figure 4-42 Results projected onto XZ plane (less than 0.125 meters)
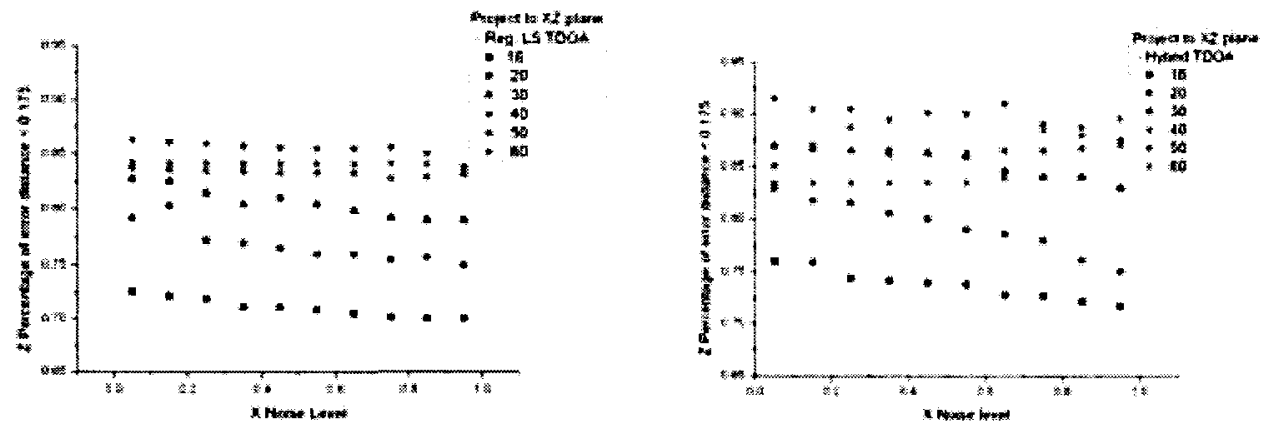

Figure 4-43 Results projected onto XZ plane (less than 0.175 meters)

In Figure 4-42 and Figure 4-43, we projected the three-dimensional figures onto the 
$\mathrm{XZ}$ plane, so the influence of density on performance can be analyzed. The four figures show that the bigger density always results in better performance at different noise levels. Furthermore, we can see that the noise level has a great impact on the performance when the density is around 16 to 30 . After the density has become very large (greater than 40), the influence of noise on the performance is minor, because the major influence is congestion, not noise, when the density is very large, as reflected in Figure 4-35.

The simulations in this section show that the density of the reference node and noise can both influence performance, and the density of the reference node contributes more to the influence than the noise does. 


\section{Chapter 5 Conclusions and Future Work}

Conclusions and proposed future work are presented as follows.

\subsection{Conclusions}

A Hybrid TDOA method was presented in this thesis. It combines the cell ID location estimation method and TDOA method, and also incorporates GDOP theory into the location estimation method. This Hybrid TDOA method is robust in noisy environments, due to the properties of the TDOA method. Moreover, the ambiguity problem is solved by combining Cell ID location estimation method. Furthermore, the location estimation accuracy is improved through the use of GDOP theory. Therefore, this Hybrid TDOA method has the advantages of both the cell ID and the TDOA methods and improves location estimation accuracy by merging GDOP theory with the location estimation process.

Specifically, the advantages in this Hybrid TDOA method are as follows:

1. The Hybrid TDOA method provides a very practical implementation in location estimation based on those minimum assumptions held in the location estimation research area. This method requires only those assumptions that can be easily satisfied in any wireless Ad Hoc/Sensor network, which makes this method very 
practical.

2. In most situations, the Hybrid TDOA method can obtain higher location estimation accuracy than other practical TDOA methods. Although this method requires only minimum assumptions, the Hybrid TDOA still obtains relatively high location estimation accuracy.

3. By employing the Cell ID method, the Hybrid TDOA method solves the ambiguity problem when only three reference nodes can be reached by the source node, without using extra assumptions. Moreover, employing the Cell ID method costs nothing in terms of networking resources, except for some memory space in each mobile node.

4. This Hybrid TDOA method performs well in non-noisy and noisy environments.

\subsection{Future Work}

In this section, the future work in the challenging field of the Hybrid TDOA method is discussed.

1. Although the performance of the Hybrid TDOA method has been evaluated through simulations, those simulations did not consider non-line-of-sight (NLOS) propagation errors, which are induced by any obstacles between the source node and the reference nodes. Those NLOS errors can delay the propagation time of $R F$ signals [53, 54, 55] at different levels, resulting in inaccurate TDOA measurements. Fundamentally, the NLOS problem is a physical layer problem. 
Different methods exist to reduce the NLOS influence in TDOA measurement that can be employed in the Hybrid TDOA method.

2. In the Hybrid TDOA method, the simplest Cell ID method is used. If no reference node can be reached by a source node, a more complex Cell ID method can be employed to provide relatively low location estimation accuracy in such situations.

3. In the Hybrid TDOA method, the GDOP theory is used to identify and delete those category No. 1 refer-triangles. However, different relative positions between the source node and the reference nodes result in different GDOP values, as described in Section 3.4.2. Deeper research into GDOP may result in a more complex weight matrix on those refer-triangles. Because the Hybrid TDOA method only sets the weight to " 0 " for those category No. 1 refer-triangles that have higher GDOP values than category No. 2 and category No. 3 refer-triangles, and because the Hybrid TDOA method sets weight " 1 " for the other two categories, a more complex weight matrix based on more accurate GDOP values is supposed to result in a more accurate location estimation than the Hybrid TDOA method can do right now. However, the problem is that exact GDOP values are very difficult to obtain. More research can be done in this field. 


\section{References}

1. T. S. Rappaport, Wireless Communications, Principles and Practice, Prentice Hall, 1996.

2. M. Chiang and G. Carlsson, "Admission Control, Power Control and QoS analyses for Ad Hoc/Sensor Wireless Networks," Proceeding of the IEEE ICC' 01, pp. 245-249, 2001.

3. "FCC Adopts Rules to Implement Enhanced 911 for Wireless Services," FCC News, CC docket no. 94-102, June 12, 1996.

4. M. I. Silventoinen and T. Rntalainen, "Mobile Station Emergency Locating in GSM," Int'l. Conference Pers. Wireless Commun., New Delhi, India, Feb. 1996.

5. C. Drane, M. Macnaughtan, and C. Scott, "Positioning GSM Telephones," IEEE Communications Magazine 36. pp. 46-54, April 1998.

6. M. Vossiek, L. Wiebking, P. Gulden, J. Wieghardt, C. Hoffmann, and P. Heide, “Wireless Local Positioning,” IEEE Microwave Magazine, vol. 4, no. 4, pp.77 86, December 2003.

7. K. J. Krizman, T. E. Biedka, and T. S. Rappaport, "Wireless Position Location: Fundamentals, Implementation Strategies, and Sources of Error," Vehicular Technology Conference, 1997 IEEE 47th, vol. 2, pp 919-923, May 1997.

8. H.-C. Chu and R.-H. Jan, "A Cell-based Location-Sensing Method for Wireless 
Networks," Wireless Communication and Mobile Computing 3, pp. 455 - 463, April 2003.

9. P. Duff and H. Muller, "Auto-calibration Algorithm for Ultrasonic Location Systems," Seventh IEEE International Symposium on Wearable Computers, White Plains, NY, October 2003.

10. P. Bahl and V. N. Padmanabhan, "Radar: An In-Building RF-Based User-Location and Tracking System," Proceeding of IEEE INFOCOM 2000, Tel Aviv, Israel, vol. 2, pp. 775-84, March 2000.

11. N. Bulusu, J. Heidemann, and D. Estrin, "GPS-less Low Cost Outdoor Localization for Very Small Devices,” IEEE Personal Communications Magazine, 7(5):28-34, October 2000.

12. D. Niculescu and B. Nath, "Ad Hoc Positioning System (APS)," Conference of IEEE Global Telecommunications Conference (GLOBECOM), volume 5, pp. 2926-2931, 2001.

13. D. Niculescu and B. Nath, "Ad Hoc Positioning System (APS) Using AOA," In Proceedings of 22nd Conference of the IEEE Communications Society (INFOCOM'03), pp. 1734-1743, 2003.

14. http://www.uswcorp.com/USWCmainpages/our.htm, last accessed 12 December 2007.

15. http://www.arundel.net/xplane/html/vor.html, last accessed 30 November 2007.

16. C. Savarese, J. Rabaey, and J. Beutel, "Locationing in Distributed Ad-Hoc Wireless 
Sensor Networks," Proceedings of the ICASSP, Vol. 4, pp. 2037-2040, May 2001.

17. C. Savarese, K. Langendoen, and J. Rabaey, "Robust Positioning Algorithms for Distributed Ad-Hoc Wireless Sensor Networks," USENIX technical annual conference, (Monterey, CA), pp. 317-328, June 2002.

18. I. Ziskind and M. Wax, "Maximum Likelihood Localization of Multiple Sources by Altering Projection," IEEE Transactions on Acoustics, Speech, and Signal Processing, vol. 36, no. 10, pp. 1553-1560, October 1988 .

19. Hung-Chi Chu and Rong-Hong Jan, "A GPS-less Self-positioning Method for Sensor Networks," Proceedings of the 11th International Conference on Parallel and Distributed Systems - Workshops (ICPADS'05), p.629-633, July, 2006

20. R. Yamasaki R, T. Tamaki, and N. Matsuzawa, "TDOA Location System for IEEE 802.11b WLAN," Wireless Communications and Networking Conference, 2005 IEEE, vol. 4, pp. 2338-2343, March 2005.

21. George A. Mizusawa, "Performance of Hyperbolic Position Location Techniques for Code Division Multiple Access," MPRG-TR-96-29, Virginia Tech, Blacksburg, VA 24061-0350, August 1996.

22. W. A. Gardner and Chih-Kang Chen, "Signal Selective Time-Difference-of-Arrival Estimation for Passive Location of Man-Made Signal Sources in Highly Corruptive Environments, Part I: Theory and Method," IEEE Transactions on Signal Processing, vol. 40, no. 5, pp. 1168-1184, May 1992.

23. W. A. Gardner, "Cyclostationary in Communications and Signal Processing," IEEE 
Press, 1994.

24. Chih-Kang Chen and W. A. Gardner, "Signal Selective Time-Difference-of-Arrival Estimation for Passive Location of Man-Made Signal Sources in Highly Corruptive Environments, Part II: Algorithms and Performance," IEEE Transactions on Signal Processing, vol. 40, no. 5, pp. 1185-1197, May 1992.

25. C. H. Knapp and G. C. Carter, "The Generalized Correlation Method for Estimation of Time Delay," IEEE Transactions on Acoustics, Proceeding of Speech and Signal, vol. ASSP-24, no. 4, pp. 320-327, August 1976.

26. G. C. Carter, “Coherence and Time Delay Estimation,” Proceeding of IEEE, vol. 75, pp. 236-255, Feb. 1987.

27. P. R. Roth, "Effective Measurements Using Digital Signal Analysis," IEEE Spectrum, vol. 8, pp. 62-70, April 1971.

28. W. R. Hahn, "Optimum Signal Processing for Passive Sonar Range and Bearing Estimation," Journal of Acoustical Society of America, vol. 58, pp. 201-207, July 1975.

29. W. R. Hahn and S. A. Tretter, "Optimum Processing for Delay-Vector Estimation in Passive Signal Analysis," IEEE Transactions on Information Theory, vol. IT-19, no.5, pp. 608-614, September 1973.

30. G. C. Carter, "Time Delay Estimation for Passive Sonar Signal Processing," IEEE Transactions on Acoustics, Speech, and Signal Processing, vol. ASSP-29, no. 3, pp. 463-470, June 1981. 
31. B. Friedlander, "A Passive Localization Algorithm and Its Accuracy Analysis," IEEE Journal of Oceanic Engineering, vol. OE-12, no. 1, pp. 234-244, January 1987.

32. J. S. Abel, "A Divide and Conquer Approach to Least-Squares Estimation," IEEE Transactions on Aerospace and Electronic Systems, vol. 26, No.1, pp. 423-427, March 1990.

33. H. Stark and J. W. Woods, Probability, Random Processes and Estimation Theory for Engineers, Prentice-Hall, Inc., 2nd edition, 1994.

34. Wang Yongcheng and Zhang Lingkun, "Position location using TDOA Measurements in Multi-Sites Modem Radar," FRINA F.A.Statrd Radar Data Processing, 2003.

35. Linghan Fei, Jinrong Yin, and Weirong Ma, "Passive Location Using TDOA Measurements In Four Sites," Radar, 2006. CIE '06. International Conference, pp. 1-4, October 2006.

36. Hyungchui Son and Janggyu Lee, "Mobile Station Location using Hybrid GPS and a Wireless Network," Vehicular Technology Conference, 2003. VTC 2003-Spring. The 57th IEEE Semiannual, Vol. 4, pp 2716 - 2720, 22-25 April 2003.

37. Michael Bocquet, Christophe Loyez, and Aziz Benlarbi-Delaï, "Enhanced-TDOA Measurement for Ad Hoc Networks Positioning," International Workshop on Wireless Ad Hoc Networks 2005 London, UK, 2005.

38. Michael Bocquet, Christophe Loyez, and Aziz Benlarbi-Delaï, "Using Enhanced-TDOA Measurement for Indoor Positioning," IEEE Microwave and 
wireless components letters, Vol. 15, No. 10, October 2005

39. J. Cartigny, D. Simplot, and I. Stojmenovic, "Localized Energy Efficient Broadcast for Wireless Networks with Directional Antennas," IFIP Annual Mediterranean Ad Hoc Networking Workshop'2002, Sardegna, Italy, 2002.

40. T. S. Rappaport, J. H. Reed, and B. D. Woerner, "Position Location Using Wireless Communications on Highways of the Future," IEEE Communications Magazine, vol. 34, no. 10, pp. 33-41, October 1996.

41. J. Kennedy et al., "Characterization of the Cellular Radio Environment," in Proceeding of $2^{\text {nd }}$ Workshop. on Smart Antennas in Wireless Mobile Commun. Stanford Univ., July 20-21, 1995.

42. J. Kennedy and M. C. Sullivan, "Direction Finding and "Smart" Antennas Using Software Radio Architecture," IEEE Communications Magazine, vol. 3, no. 5, pp. 62-68, May 1995.

43. W. H. Foy, "Position-Location Solutions by Taylor-Series Estimation," IEEE Transactions on Aerospace and Electronic Systems, vol. AES-12, pp. 187-194, March 1976.

44. H. C. Schau and A. Z. Robinson, "Passive Source Localization Employing Intersecting Spherical Surfaces from Time-of-Arrival Differences," IEEE Transactions on Acoustics, Speech, and Signal Processing, vol. ASSP-35, no. 8, pp. 1223-1225, August 1987.

45. Y. T. Chan and K. C. Ho, "A Simple and Efficient Estimator for Hyperbolic 
Location," IEEE Transactions on Signal Processing, vol. 42, no. 8, pp. 1905-1915, August 1994.

46. B. T. Fang, "Simple Solutions for Hyperbolic and Related Fixes," IEEE Transactions on Aerospace and Electronic Systems, vol. 26, no. 5, pp. 748-753, September 1990.

47. IEEE 802.11b WG, Part 11, "Wireless LAN Medium Access Control (MAC) and Physical Layer (PHY) specification: High-speed Physical Layer Extension in the 2.4 GHz Band," IEEE, September 1999.

48. E.W. Anderson, Principles of Navigation, Hollis \& Carter, London, 1966.

49. Chin-Der Wann and Ming-hui Lin, "Location Estimation with Data Fusion for Wireless Location Systems," Networking, Sensing and Control, 2004, IEEE International Conference, Vol. 1, pp. 327-332, March 2004.

50. P. Massatt and K. Rudnick, "Geometric Formulas for Dilution of Precision Calculations," Journal of the Institute of Navigation, Vol. 37, pp. 379-391, 1991.

51. J. L. Caffery, Wireless Location in CDMA Cellular Radio Systems, Kluwer Academic, 2000.

52. N. Levanon, "Lowest GDOP in 2-D scenarios," Proceedings of Radar, Sonar and Navigation, IEEE, Vol. 147, pp. 149-155, June 2000.

53. S.S. Woo, H.R. You, and J.S. Koh, "The NLOS Mitigation Technique for Position Location using IS-95 CDMA Networks," Proceeding of Vehicular Technology Conf. Boston, MA, vol. 6, pp. 2556-2560, 2000.

54. Li Cong and Weihua Zhuang, "Nonline-of-Sight Error Mitigation in TDOA Mobile 
Location," Global Telecommunications Conference, 2001. GLOBECOM '01. IEEE, pp. 680-684, Vol. 1, December 2001.

55. Li Cong and Weihua Zhuang, "Nonline-of-Sight Error Mitigation in Mobile Location," Wireless Communications, IEEE Transactions on, Vol. 4, pp. 560- 573, Issue: 2, March 2005.

56. Eitan Altman and Tania Jimenez, "NS Simulator for beginners". http://www-sop.inria.fr/maestro/personnel/Eitan.Altman/COURS-NS/n3.pdf, last accessed 5 April 6, 2008.

57. J. Heidemann, N. Bulusu, J. Elson, C. Intanagonwiwat, K. chan Lan, Y. Xu, W. Ye, D. Estrin, and R. Govindan, "Effects of Detail in Wireless Network Simulation". Proceedings of the SCS Multiconference on Distributed Simulation, pp. 3-11, Phoenix, Arizona, January 2001.

58. Polly Huang, Deborah Estrin, and John Heidemann, "Enabling Large-Scale Simulations: Selective Abstraction Approach to the Study of Multicast Protocols". Proceedings of the International Symposium on Modeling, Analysis and Simulation of Computer and Telecommunication Systems, pp. 241-248, Montreal, Canada, July 1998.

59. K. Pawlikowski, H.-D. Jeong, and J.-S. Lee, "On Credibility of Simulation Studies of Telecommunication Networks". IEEE Communications, 40(1), pp. 132-139, January 2002.

60. K. Fall and K. Varadhan, "The ns manual". http://www.isi.edu/nsnam/ns/doc/index.html, 
last accessed 4 April, 2008.

61. J. Banks and J.S. Carson, Discrete Event System Simulation, Prentice-Hall, Englewood Cliffs, N.J. 1984.

62. Computational complexity of mathematical operations, http://en.wikipedia.org/wiki/Computational_complexity_of_mathematical_operations \#Matrix_algebra, last accessed 11 May 2008. 


\section{Appendix A: Triangulation}

In trigonometry and geometry, triangulation is the process of finding out the coordinates and distance to a point by calculating the length of one side of a triangle, given measurements of angles and sides of the triangle formed by that point and two other known reference points, using the law of sines.

In wireless location identification systems, the term "triangulation" is a process based on the basic definition in the paragraph above, in which the location of a radio transmitter can be located by measuring either the radial distance or the direction of the received signal from two or three different points. 


\section{Appendix B: Cross-correlation Technique}

To estimate accurate TDOA measurements, cross-correlation techniques are used because they provide resistance at certain levels to noise and interference and can partially resolve multi-path signal components.

For a certain $R F$ signal, noted as $s(t)$, which radiates from a source mobile node with interference and noise, the general equations for the time-delay estimation between signals at two reference nodes, noted $x_{1}(t)$ and $x_{2}(t)$, can be given by:

$$
\begin{aligned}
& x_{0}(t)=s(t)+n_{0}(t) \\
& x_{1}(t)=a_{1}\left(t-\Delta t_{1}\right)+n_{1}(t)
\end{aligned}
$$

Where $a_{1}$ is the amplitude ratio between the amplitude scaling of the $R F$ signal, $n_{0}(t)$ and $n_{1}(t)$ consist of noise and interfering RF signals. Estimating $\Delta t_{i}$ is desired, and it may also be desirable to estimate the amplitude scaling $a_{1}$. The method for estimating the amplitude scaling involves many low-layer physical knowledge and techniques; interested readers can refer to [22].

In cross-correlation techniques, different methods are developed to estimate $\Delta t_{i}$ with varying degrees of accuracy and robustness. These include the Generalized Cross-Correlation (GCC) $[22,23]$ and Cyclic Cross-Correlation (CCC) methods $[22,23]$. The Cyclic Cross-Correlation (CCC) methods include several sub-methods. They are the 
Spectral-Coherence Alignment (SPECCOA) method [22, 23], the Cyclic Cross-Correlation (CYCCOR) method [22], the Cyclic Prony method [23], and the Band-Limited Spectral Correlation Ratio (BL-SPECCORR) method [22]. In this thesis, detailed discussion on those methods is not included. Interested readers can refer to the papers and books mentioned above.

In $[22,23]$, the authors proved that the Cyclic Cross-Correlation methods perform better than the GCC method in the presence of noise and interference. However, that better performance has a precondition that spectrally overlapping noise and interference exhibit a cycle frequency different from the signal of interest [22]. But if spectrally overlapping signals exhibit the same cycle frequency, those methods do not have any advantage over the GCC method. Therefore, the selection of the Cross-Correlation method can be based on a certain network scenario. In this thesis, no detailed physical $R F$ signal is considered. The simplest cross-correlation method is employed to provide the TDOA measurements. 


\section{Appendix C: Simulation Implementation in NS2}

NS2 is the second release of the NS (network simulator) that began as a variant of the REAL network simulator (a network simulator originally intended for studying the behaviors in packet-switched data networks) in 1989 and has evolved substantially over the last few years. NS2 is an object-oriented simulator. NS2 is used extensively in the networking research community. NS2 is an event-driven and non-real-time core coded with $\mathrm{C}++$. It employs $\mathrm{Tcl}$ and Object $\mathrm{Tcl}$ as interface and loads script files to describe the setup and model to carry out the simulation [60].

\section{Physical Layer Setting}

The physical layer setting is important in this simulation. In the MAC layer, the IEEE802.11 protocols are employed. The IEEE802.11 settings are as follows:

\# The default is changed to 1

Mac/802_11 set bugFix_timer_true; \# default changed 2006/1/30

Mac/802_11 set CWMin__ 15

Mac/802_11 set CWMax__ 1023

Mac/802_11 set SlotTime_ 0.000009 ;\# 9us CHECK

Mac/802_11 set SIFS_ $\quad 0.000010 \quad ; \# 10$ us CHECK 


$$
\begin{aligned}
& \text { Mac/802_11 set PreambleLength_ } 72 \text {;\# CHECK } \\
& \text { Mac/802_11 set PLCPHeaderLength_ } 48 \text {;\# CHECK } \\
& \text { Mac/802_11 set PLCPDataRate_ 6.0e6 ; ; 6Mbps } \\
& \text { Mac/802_11 set basicRate_6.0e6; }
\end{aligned}
$$

The antenna of the terminals in wireless networks has a strong influence on the physical layer. Therefore, the Antenna setting is also specified, as follows.

\# unity gain, omni-directional antennas

\# set up the antennas to be centered in the node and 1.5 meters above it

Antenna/OmniAntenna set $X_{-} 0$

Antenna/OmniAntenna set $\mathrm{Y}_{-} 0$

Antenna/OmniAntenna set $Z_{-} 1.5$

Antenna/OmniAntenna set Gt_19.953;

Antenna/OmniAntenna set Gr_19.953;

\section{Noisy Propagation Model}

This section describes the Radio propagation model and setting in the simulation in this thesis. Detailed information on those models can be found in [56].

In NS2, three propagation models are implemented. They are the Free Space model, the Two-ray Ground Reflection model, and the Shadowing model. These models are used to simulate the received signal power of each packet. At the physical layer of each 
wireless node, a receiving threshold exists. When a packet is received, if its signal power is below the receiving threshold, it is marked as an error and dropped by the MAC layer.

\section{Free Space model}

The Free Space propagation model deploys the ideal propagation condition that there is only one clear line-of-sight path between the transmitter and the receiver. This model uses the following equation to calculate the received signal power in free space at distance $d$ from the transmitter.

$$
P_{r}(d)=\frac{P_{t} G_{t} G_{r} \lambda^{2}}{(4 \pi)_{2} d^{2} L}
$$

$P_{t}$ is the transmitted signal power. $G_{t}$ and $G_{r}$ are the antenna gains of the transmitter and the receiver respectively. $L$ is the system loss, and $\lambda$ is the wavelength. Typically, most simulations select $G_{t}=G_{r}=1$ and $L=1$ in NS2. Broadly speaking, the Free Space model represents the radio transmission range as a circle around the transmitter. If a receiver is within the circle, it receives all packets. Otherwise, it loses all packets. This is the simplest propagation in NS2.

\section{Two-ray Ground Reflection model}

In the Two-ray Ground Reflection model, the single line-of-sight path between any two nodes is seldom the only means of propagation. This model takes both the direct path and a ground reflection path into consideration. Other research shows that this model provides more accurate predictions at a long distance than the Free Space model [34]. The received power at distance $d$ is predicted by 


$$
P_{r}(d)=\frac{P_{t} G_{t} G_{r} h_{t}{ }^{2} h_{r}{ }^{2}}{d^{4} L}
$$

$h_{t}$ and $h_{r}$ are the heights of the transmit and receive antennas respectively. Typically, we assume $L=1$. To be consistent with the free space model, $L$ is added here. The above equation shows a quicker power loss than the equation used in the Free Space model when distance increases. However, because the destructive and constructive combination of the two rays causes oscillation, the Two-ray model does not give good results over a short distance. In the situation where the distance is short ( $d$ is small), the Free Space model is still deployed.

In order to deal with this problem, a cross-over distance $d_{c}$ is calculated. If $d<d_{c}$, this model still deploys the free model equation. If $d>d_{c}$, it uses its own equation. By deploying the cross-over distance, the two equations gain the same result. And $d_{c}$ is calculated in the following equation:

$$
d_{c}=\left(4 \pi h_{t} h_{r}\right) / \lambda
$$

\section{Shadowing Model}

The Free Space model and the Two-ray model simulate the received power as a deterministic function of distance. The radio transmission range in both is an ideal circle. In the real world, however, because of the multi-path propagation effects, the received power at different distances is a random variable. In fact, the previous two models just reflect the mean power at a certain distance $d$. The third model implemented in NS2, the Shadowing model [34], is more general and widely used. The Shadowing model consists 
of two parts. The first is a path loss model, which predicts the mean received power at distance $d$, denoted by $\overrightarrow{P_{r}(d)}$. It uses a close-in distance $d_{0}$ as a reference. $\overline{P_{r}(d)}$ is computed relative to $\operatorname{Pr}\left(d_{0}\right)$, as follows.

$$
\frac{p_{r}\left(d_{0}\right)}{p_{r}(d)}=\left(\frac{d}{d_{0}}\right)^{\beta}
$$

$\beta$ is the path loss exponent, and is usually empirically determined by field measurement. From the equation of the Free Space model, we know that $\beta=2$ for free space propagation. The NS2 manual gives some typical values of $\beta$, shown in the table below. Larger values correspond to more obstructions and hence a faster decrease in the average received power as the distance becomes larger. From the equation of the Free Space model, $P_{r}\left(d_{0}\right)$ can be computed.

Table A-0-1 Some typical values of path loss exponent $\beta$

\begin{tabular}{|l|l|l|}
\hline \multicolumn{2}{|l|}{ Environment } & $\beta$ \\
\hline \multirow{2}{*}{ Outdoor } & Free space & 2 \\
\cline { 2 - 3 } & Shadowed urban area & $2.7 \sim 5$ \\
\hline \multirow{2}{*}{ In building } & Line of sight & $1.6 \sim 8$ \\
\cline { 2 - 3 } & obstructed & $4 \sim 6$ \\
\hline
\end{tabular}

The path loss is measured in $d B$. Therefore, from the above equation, the following is obtained:

$$
\left[\frac{\overline{p_{r}(d)}}{p_{r}\left(d_{0}\right)}\right]_{d B}=-10 \beta \log \left(\frac{d}{d_{0}}\right)
$$


The second part of the Shadowing model is used to reflect the variation of the received power at a certain distance. It is a log-normal random variable. It is of Gaussian distribution if measured in $d B$. The overall Shadowing model is represented by the following equation:

$$
\left[\frac{p_{r}(d)}{p_{r}\left(d_{0}\right)}\right]_{d B}=-10 \beta \log \left(\frac{d}{d_{0}}\right)+X_{d B}
$$

$X_{d B}$ is a Gaussian random variable with zero mean and standard deviation $\delta_{d B}{ }^{*} . \delta_{d B}$ is called the Shadowing deviation. The following table from [34] shows some typical values of $\delta_{d B}$.

Table A-0-2 Environment of $\delta_{d B}(\mathrm{~dB})$

\begin{tabular}{|c|c|}
\hline Environment & $\delta_{\mathrm{dAB}}(\mathrm{dB})$ \\
\hline Outdoor & $4 \sim 12$ \\
\hline Office, hard partition & 7 \\
\hline Office, soft partition & 9.6 \\
\hline Factory, line of sight & $3 \sim 6$ \\
\hline Factory, obstructed & 6.8 \\
\hline
\end{tabular}

In the Shadowing model, nodes can only probabilistically communicate if near the edge of the communication range, which make it a rich statistical model compared to the other two models. This is the best model for use in this thesis, because it can simulate the real world more realistically than the other two. 


\section{Appendix D: Hybrid TDOA Location Identification Method Implementation Details}

\section{Data receiving portion:}

The data receiving part is used to obtain and percolate the information from the NS2 simulator in order to provide the necessary data for location estimation process in hybrid TDOA method (mostly implemented in function "fileAnalyze" in the source code). This part is mainly used to produce the following data by percolating the NS2's output:

- The accurate location of the source node at the time point when the location measurement occurs

- The accurate locations of the reference nodes at the time point when the location measurement occurs

- Calculation of time differences from a set of three reference nodes by obtaining the information from the MAC layer trace file in NS2

\section{Estimation portion:}

The TDOA measurements in NS2 are collected from the data receiving portion. In this part, the final estimation is made. The implementation follows the algorithm described in Section 3.5.1. For the regular TDOA, the implementation simply follows the equations in Section 4.2.3. 
The process flow of the Hybrid TDOA estimation part is briefly described in the following flow chart:

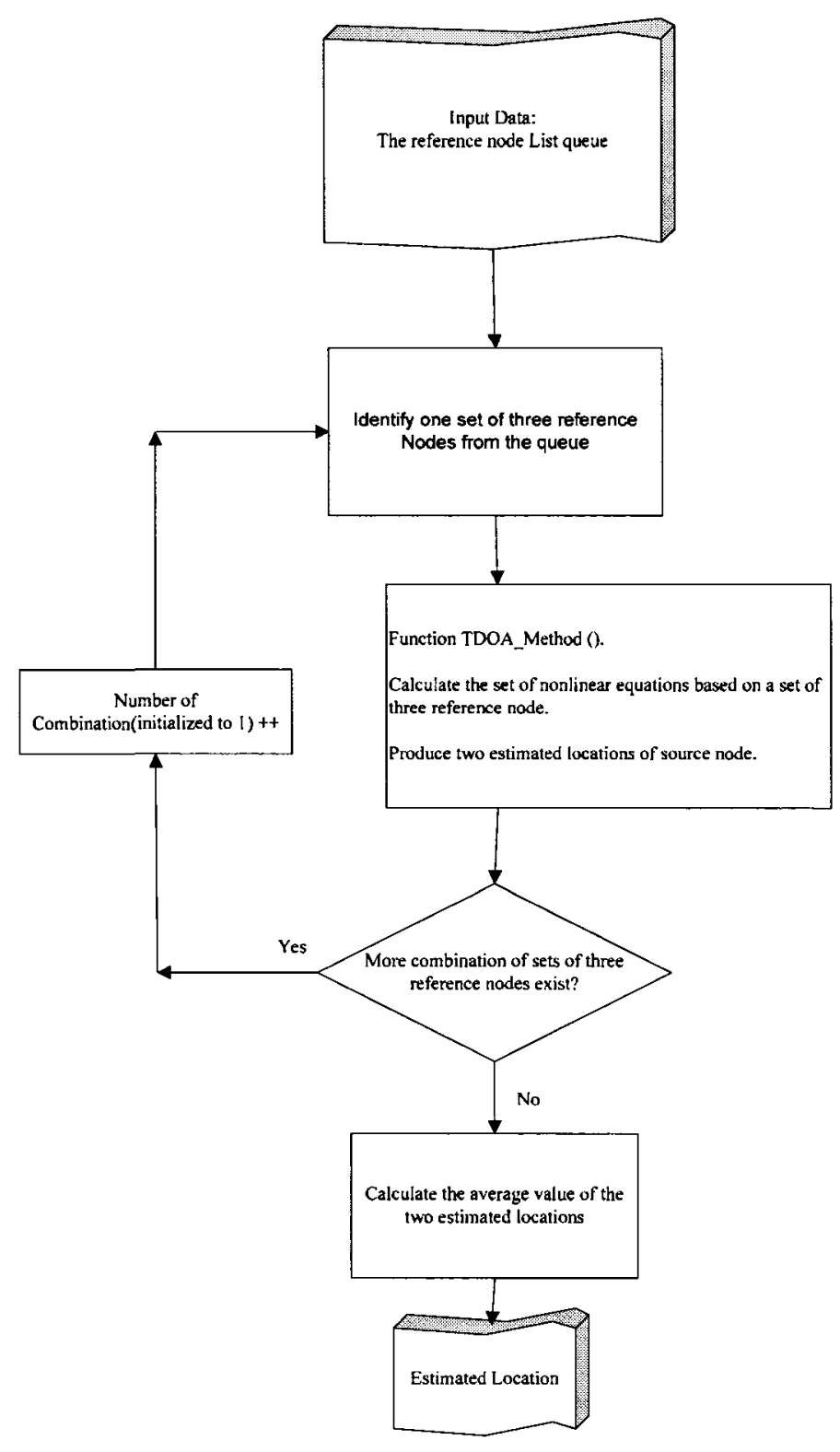

Figure C-0-1 TDOA calculation program models

\section{Cell ID method portion:}

The Cell ID method is explained in this portion. The functionalities of this part are also described in this section and a flow chart is given at the end of this section. 
This part is, in fact, a rough location identification method that exists in cellular systems (GSM, CDMA) plus assistant functionalities for TDOA measurement. The Cell ID method is also employed in Ad HoclSensor networks as an easy and very low cost implementation for roughly estimating the location of mobile nodes. In the hybrid TDOA algorithm, it is used to assist the TDOA method to increase estimation accuracy and reduce cost. The following description will hold the assumption that the hybrid TDOA method is employed. Otherwise, the following process will be ignored.

The main function is called "Cell_Method", which will be called before the TDOA method part is called. This function will first re-flash the reference node list. Then, following the Cell ID method, based on the radio coverage and location information of the reference nodes, the rough location of source node can be identified. Finally, based on the relative locations of the source node and the reference nodes, some reference nodes may be deleted from the queue of the structure "_Cell_Info_".

After the TDOA method part has been called, two estimated values will be derived. The Cell ID method part will calculate the distances between the estimated location and all reference nodes that received the source node signal. One of the two locations must be beyond a signal scope of some reference node. If both are beyond the signal scope of some reference node, there must be some obstacle between the source node and the reference node (NLOS situation).

The process flow of the cell ID method part is briefly described in the following flow chart: 


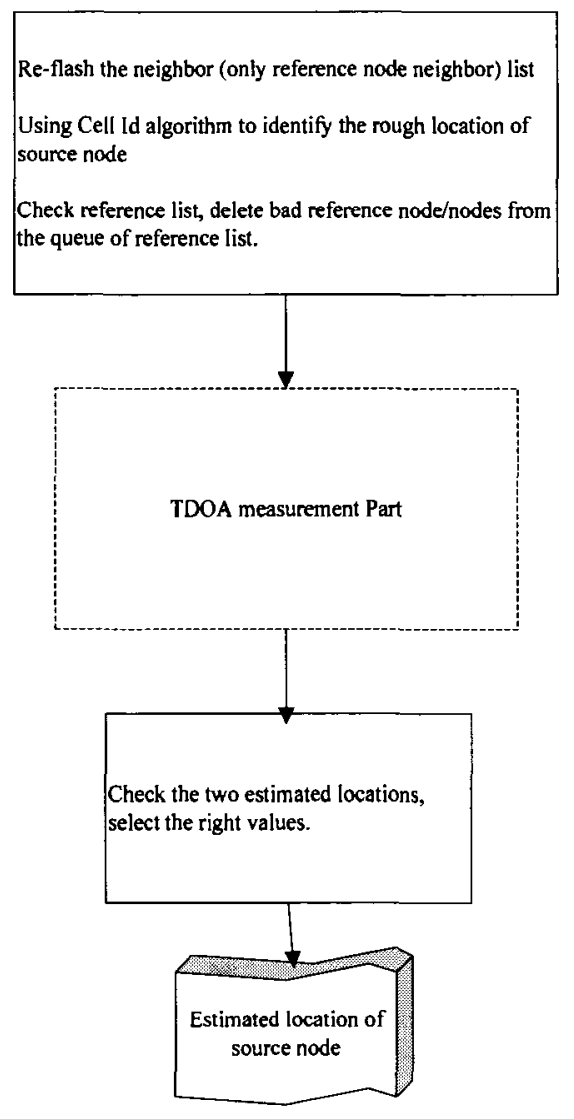

Figure C-0-2 Cell ID method program models

\section{Pseudo Code of Deleting Category No. 1 refer-triangle}

In this pseudo code, the following assumptions and statements are held:

Assuming the estimated coordinates of source node is $(x, y)$;

The coordinates of reference node 0 are $(0,0)$;

The coordinates of reference node 1 are $(x 1,0)$;

The coordinates of reference node 2 are $(x 2, y 2)$;

Assuming the slope of the line connecting reference nodes 1 and 2 is $S(1-2)$, then 


$$
S(1-2)=\frac{0-y 2}{x 1-x 2}
$$

Assuming the slope of the line connecting reference node 1 and the source node is $\mathrm{S}(1-\mathrm{S})$, then

$$
S(1-S)=\frac{0-y}{x 1-x}
$$

Assuming the slope of the line connecting reference node 0 and 2 is $S(0-2)$, then

$$
S(0-2)=\frac{y 2}{x^{2}}
$$

Assuming the slope of the line connecting reference node 0 and source node is $S(0-S)$,

then

$$
S(0-S)=\frac{y}{x}
$$

\section{Pseudo code of the algorithm on identifying refer-triangle type:}

IF y $\times$ y $2<0\{/ /$ Source node and node 2 are on different sides of $X$ coordinate

The refer-triangle $\in$ category No. 1;

Return;

\}$/ /$ End of $($ IF $y \times y 2<0)$

IF $y>0\{$

// the source node and reference node 2 are located in quadrant I or II;

IF $\mathrm{x}>\mathrm{x} 1$ AND $\mathrm{x} 2<\mathrm{x} 1\{$

// Node 2 is left side of node 1 , and node 1 is left side of source node

The refer-triangle $\in$ category No. 1;

Return; 
\} ELSE //Else of (IF $x>x 1$ AND x $2<x 1$ )

IF $x>0$ AND $x 2>0$

//Source node and node 2 are both in quadrant I

IF $S(1-S)<S(1-2) \quad\{$

//the slope of the line from node 1 to source node is higher

//than that of the line from node 1 to node 2

The refer-triangle $\in$ category No. 1;

Return;

\}ELSE //Else of (IF S(1-S) $<\mathrm{S}(1-2))$

IF $\mathrm{S}(0-\mathrm{S})>\mathrm{S}(0-2) \quad\{$

// the slope of the line from node 0 to source node is higher

//than that of the line from node 0 to node 2

The refer-triangle $\in$ category No. 1;

Return;

\} ELSE $\{/ /$ Else of IF $\mathrm{S}(0-\mathrm{S})>\mathrm{S}(0-2))$

The refer-triangle doesn't belong to category No. 1;

Return;

\} //End of ELSE (IF S(0-S) $>\mathrm{S}(0-2))$

\}/End of (IF S(1-S) $<\mathrm{S}(1-2))$

\}$/ /$ End of (IF x $>0$ AND x2 >0)

IF $\mathrm{x}>0$ AND $\mathrm{x} 2<0\{$ 
//Source node is in quadrant I and node 2 is in quadrant II

IF $S(1-2)>S(1-S) \quad\{$

// the slope of the line from node 1 to source node is higher than that

//of the line from node 1 to node 2

The refer-triangle $\in$ category No. 1 ;

Return;

\} ELSE //Else of (IF S $(1-2)>\mathrm{S}(1-\mathrm{S}))\{$

The refer-triangle doesn't belong to category No. 1;

Return;

\}$/ /$ End of ELSE (IF S(1-2) > S(1-S))

IF $\mathrm{x}<0$ AND $\mathrm{x} 2>0$

// Source node is in quadrant II and node 2 is in quadrant I

The refer-triangle $\in$ category No. 1;

Return;

\}$/ /$ End of $(\mathrm{IF} \mathrm{x}<0$ AND $\mathrm{x} 2>0)$

IF $\mathrm{x}<0$ AND $\mathrm{x} 2<0$

// Source node and node 2 are both in quadrant II

IF $\mathrm{x}>\mathrm{x} 2$

//Source node is on the right side of node 2

IF $S(1-2)>S(1-S) \quad\{$

// the slope of the line from node 1 to source node is 
//higher than that of the line from node 1 and node 2

The refer-triangle $\in$ category No. 1;

Return;

\}ELSE //Else of (IF S(1-2) > S(1-S)) \{

IF $\mathrm{S}(0-2)<\mathrm{S}(0-\mathrm{S})$

$/ /$ the slope of the line from node 0 to source node is

//lower than that of line from node 0 to node 2

The refer-triangle $\in$ category No. 1;

Return;

\} ELSE //Else of $(\mathrm{IF} \mathrm{S}(0-2)<\mathrm{S}(0-\mathrm{S}))\{$

// the slope of the line from node 0 to source node is

//higher than that of the line from node 0 to node 2

The refer-triangle doesn't belong to category No. 1;

Return;

\} //End of ELSE (IF S(0-2) $<\mathrm{S}(0-\mathrm{S}))$

\} //End of ELSE (IF S(1-2) > S(1-S))

\} ELSE //Else of (IF x $>$ x2)

The refer-triangle $\in$ category No. 1;

Return;

\} //End of ELSE (IF $x>x 2)$

\} //End of (IF $x<0$ AND x $<<$ ) 


$$
\begin{aligned}
& \text { \}//End of (IF } x>0 \text { AND x2 }<0 \text { ) } \\
& \text { \} //End of (IF } x>x 1 \text { AND } x 2<x 1) \\
& \} / / \text { End of (IF } y>0) \\
& \text { END of pseudo code. }
\end{aligned}
$$

The following two examples demonstrate how the algorithm works.

The relative position between the source node and the refer-triangle is illustrated in Figure D-3.

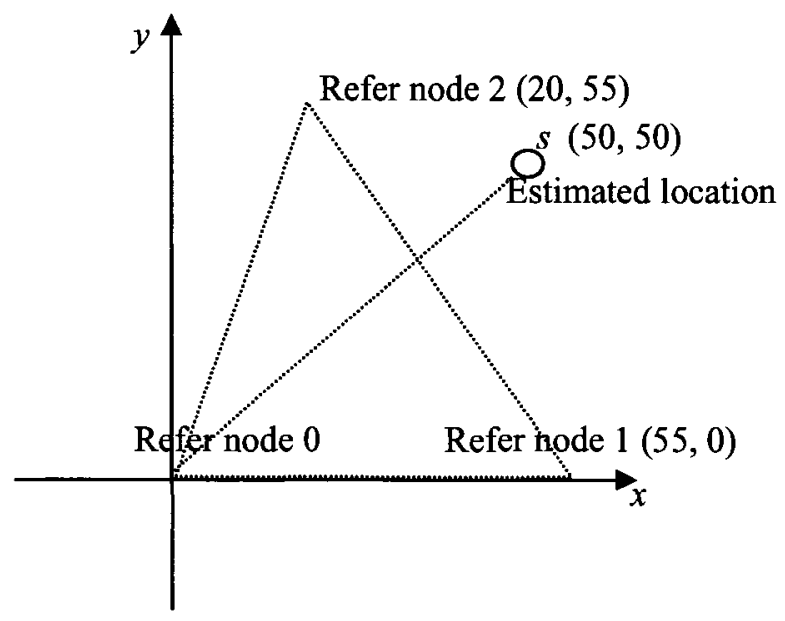

Figure D-3 Pseudo code example 1

The three reference nodes are noted as: $\mathrm{R} 0(0,0), \mathrm{R} 1(55,0), \mathrm{R} 2(20,55)$, and the source node is noted as $s(50,50)$. Then the following results are obtained:

$$
\begin{aligned}
& S(1-2)=-1.83 \\
& S(1-s)=-10 \\
& S(0-2)=2.75 \\
& S(0-s)=1
\end{aligned}
$$

The following illustrates the pseudo code, step by step: 
IF $50 \times 55<0\{$

The refer-triangle belongs to category No. 1;

Return;

\}//End of (IF $50 \times 55<0)$

IF $50>0\{$

The source node and reference node 2 are located in quadrant I or II;

IF $50>55$ AND $20<55$

The refer-triangle $\in$ category No. 1;

Return;

\} ELSE //Else of (IF $50>55$ AND $20<55$ )

$$
\begin{gathered}
\text { IF } 50>0 \text { AND } 20>0 \\
\text { IF }-10<-1.83
\end{gathered}
$$

The refer-triangle $\in$ category No. 1;

Return;

In "IF $-10<-1.83$ ", the pseudo code returns that the relative position belongs to category No. 1 . Therefore, the estimation will not be accepted.

Let's see another example.

The relative position between the source node and the refer-triangle is illustrated in Figure D-4. 


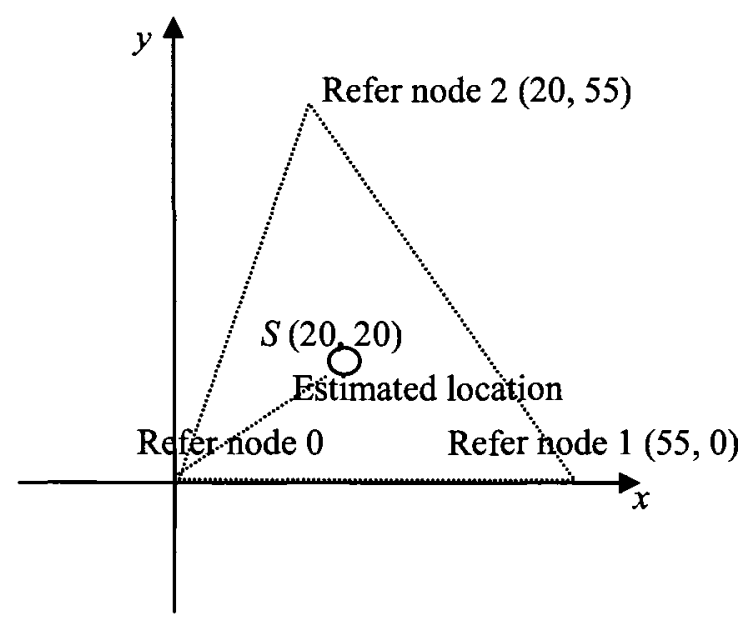

Figure D-4 Pseudo code example 2

The three reference nodes are noted as: $\mathrm{R} 0(0,0), \mathrm{R} 1(55,0), \mathrm{R} 2(20,55)$, and the source node is noted as $s(20,20)$. Then the following results are obtained:

$$
\begin{aligned}
& S(1-2)=-1.83 \\
& S(1-s)=-0.57 \\
& S(0-2)=2.75 \\
& S(0-s)=1
\end{aligned}
$$

Again, the following demonstrates the pseudo code in sequence:

IF $20 \times 55<0\{$

The refer-triangle $\in$ category No. 1;

Return;

\}

IF $20>0\{$

//The source node and reference node 2 are located in quadrant I or II; 
IF $20>55$ AND $20<55$

The refer-triangle $\in$ category No. 1 ;

Return;

\} ELSE //Else of (IF $20>55$ AND 20 <55)

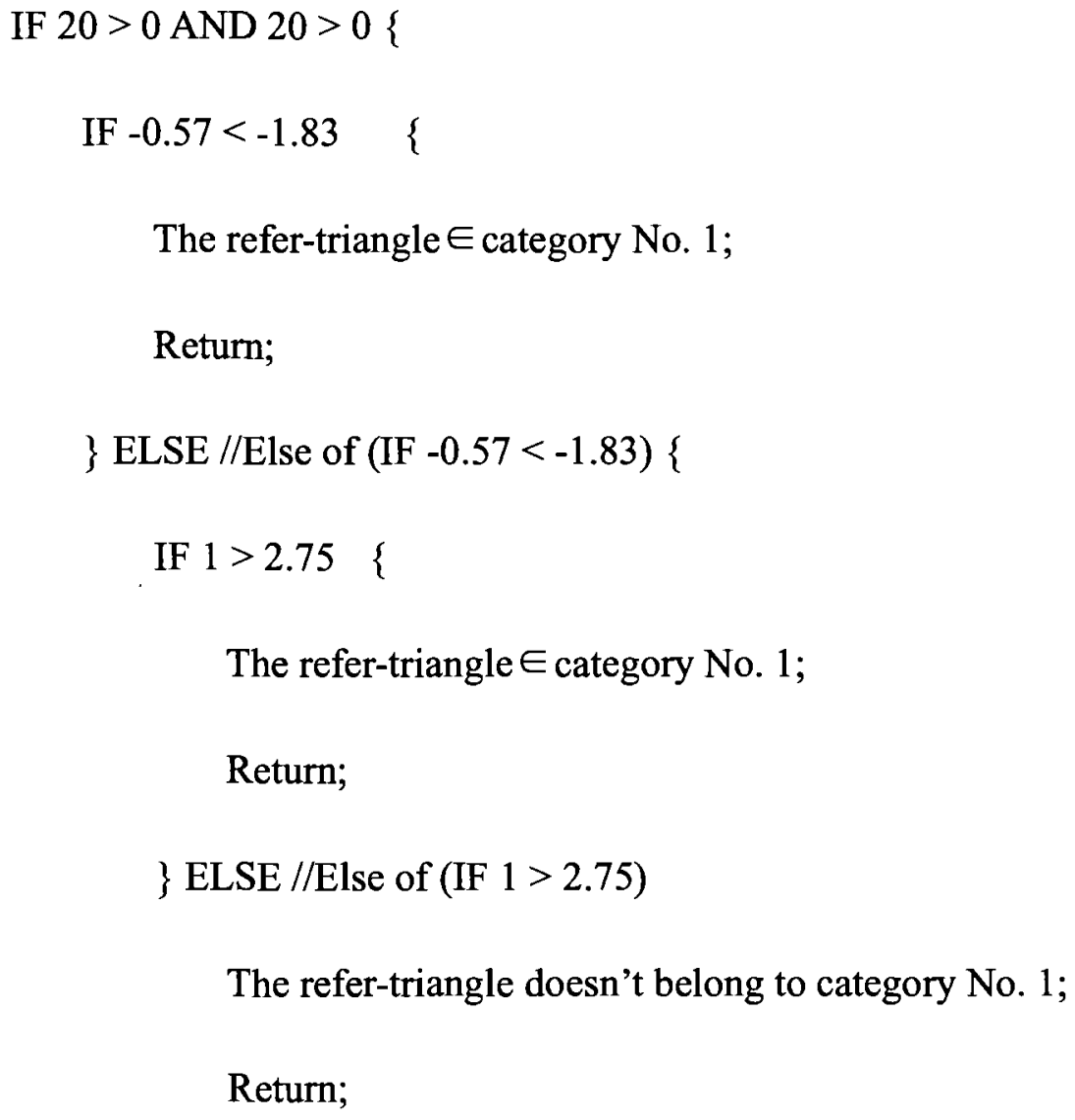

In "ELSE //Else of (IF $1>2.75$ )", pseudo code returns that the relative position does not belong to category No. 1, and the estimation will be included in the calculation. 